\title{
Evolution récente de l'appareillage et des techniques de mesures en aérodynamique et en hydrodynamique
}

\author{
New developments concerning aerodynamics and hydrodynamics \\ measurement techniques and equipment
}

\author{
J. Paulon
}

ONERA

Au cours des dernières années, des moyens de mesure nouveaux et très performants ont été développés et mis au point dans divers laboratoires et centres de recherches.

Le principe de ces nouveaux moyens est brièvement exposé et des exemples d'application sont donnés, particulièrement dans le domaine de l'aérodynamique ainsi que dans celui de l'hydrodynamique.

Ce tour d'horizon, certes incomplet, montre que les chercheurs disposent d'un large éventail de moyens de mesure permettant d'accéder à une connaissance plus fine des phénomènes.

In recent years, new and high-performance measurement means have been developed and used in various laboratories and research centres.

The principle relating to these new means is briefly described and application examples are given, in particular as regards the aerodynamics and hydrodynamics fields.

This innovation shows that the research workers have a wide range of measurement means enabling more precise knowledge of such phenomena to be acquired.

\section{Introduction}

Au cours des dernières années, les modèles mathématiques d'analyse et de prévision ont connu un développement considérable dans de nombreux domaines tels que ceux qui nous intéressent, à savoir : l'air et l'eau.

Pour certains spécialistes, il semble aujourd'hui que les outils mathématiques sont en avance sur les données physiques représentatives indispensables à la mise au point et au développement de ces modèles. Si cela est vrai dans certains domaines, il faut toutefois signaler le développement, également considérable, de moyens de mesure nouveaux, très performants, en cours d'utilisation ou de mise au point dans certains centres de recherches et laboratoires.

Le but de cet exposé est de faire un tour d'horizon, malheureusement incomplet, sur les techniques développées au cours des dernières années. Il ne faudrait toutefois pas se persuader que l'emploi de ces moyens qui vont vous être présentés a pour corollaire la mise au rebut des méthodes classiques utilisées partout depuis des dizaines d'années. Ces dernières sont et resteront toujours indispensables tandis que les plus récentes, complémentaires, permettant une analyse plus détaillée et plus fine des phénomènes, ont des possibilités d'accès plus larges et moins contraignantes, mais demandent plus de soin, de préparation, tout en étant souvent onéreuses. 


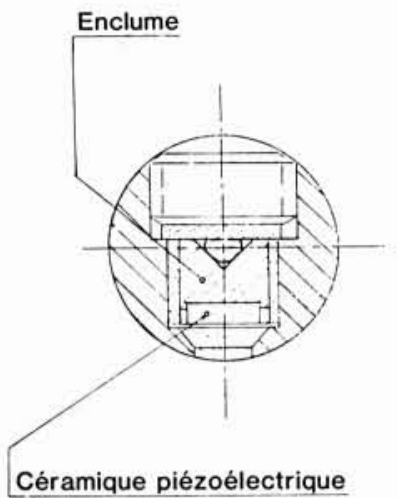

1. Principe du capteur piézoélectrique.

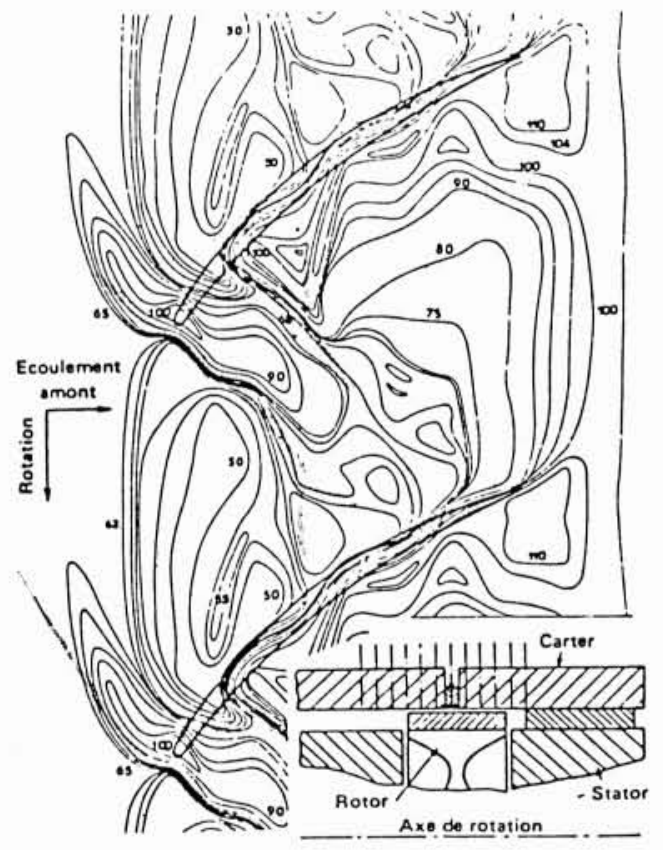

3. Champ d'isobares au droit d'une roue mobile de compresseur supersonique. Localisation des ondes de choc.

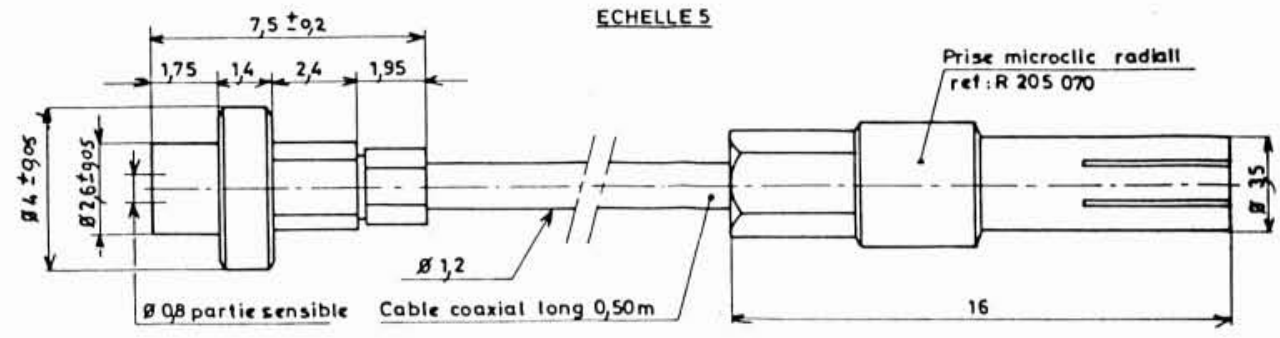

2. Vue d'un capteur $20 \mathrm{H} 48$.
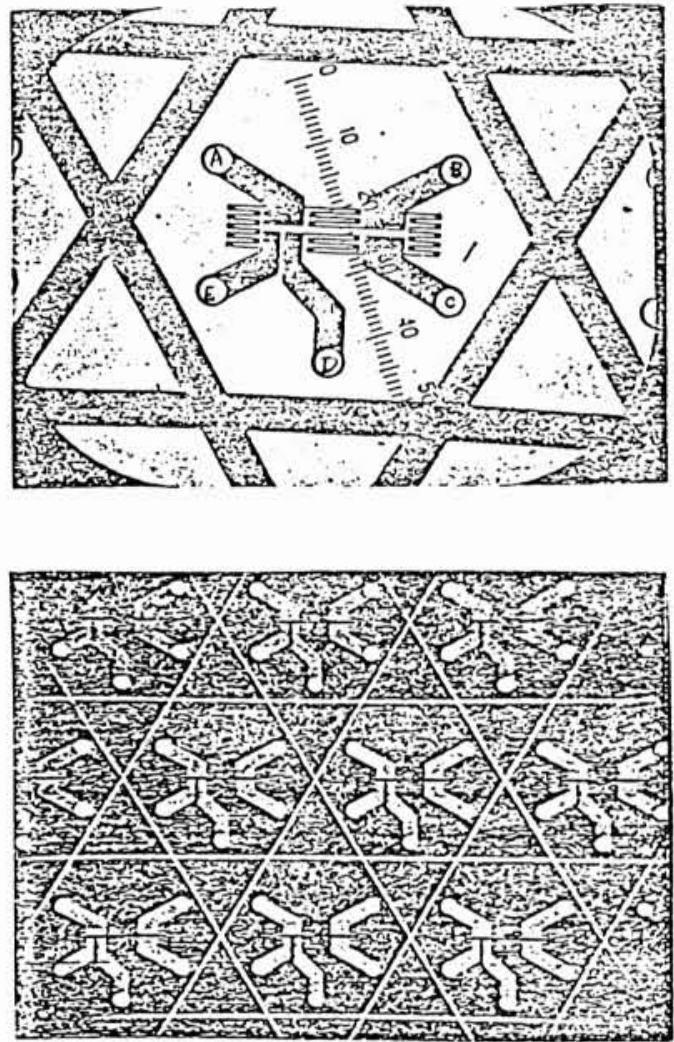

4. Capteurs à jauges intégrées.
En ce qui concerne la présentation de ces méthodes récentes, nous avons choisi d'indiquer les divers moyens permettant d'accéder à la mesure de tel ou tel paramètre ou information selon le schéma du tableau $l$ ci-après : 
Tableau I

\begin{tabular}{|l|}
\hline \multicolumn{1}{c|}{ Paramètre ou information } \\
\hline 2 - Pression \\
3 - Vitesse \\
4 - Débit \\
5 - Température \\
6 - Hauteur d'eau \\
7 - Qualité de l'eau \\
8 - Diphasique \\
9 - Visualisation \\
10 - Transmission acquisition et traitement des donnèes
\end{tabular}

\section{Mesures de pression}

Si l'emploi des capteurs est maintenant très largement répandu pour la mesure des pressions stationnaires, de nouveaux capteurs ont été développés au cours des dernières années, qui permettent de mesurer les pressions instationnaires et qui d'autre part ont été miniaturisés au point de pouvoir être implantés sur ou dans des parois aussi minces que des profils de ventilateur ou de compresseur.

Nous nous intéresserons donc particulièrement à ces nouveaux capteurs selon le classement indiqué dans le tableau II ci-dessous :

Tableau II

\begin{tabular}{|ll|}
\hline & Capteurs pour mesures de pression \\
\hline $2-1$ & Capteurs piézoélectriques \\
$2-2$ & Capteurs piézorésistifs \\
$2-3$ & Capteurs pelliculaires \\
\hline
\end{tabular}

\subsection{Capteurs piézoélectriques :}

Ces capteurs se composent d'un disque de céramique au zirconate de plomb qui fait apparaitre sur ses électrodes des charges électriques sous l'effet de contraintes mécaniques [1].

Ce disque est cimenté sur une enclume en cuivre reliée au point chaud du montage électronique; sa face supérieure est à la masse du boîtier par l'intermédiaire d'une couche de ciment conducteur (fig. 1).

Ce type de capteur permet de mesurer les fluctuations de pression dans une gamme très étendue de fréquence avec un niveau élevé de sortie. Au point de vue chaîne de mesure, ils doivent être suivis d'un adaptateur d'impédance ou d'un amplificateur de charge. A titre d'exemple la figure 2 montre un tel capteur du type $20 \mathrm{H} 48$ développé à l'ONERA et commercialisé par LEM; le tableau $I I I$ en indique les principales caractéristiques :

De tels capteurs sont très commodes pour déterminer le champ de pression instationnaire au niveau d'une paroi et mettre par exemple en évidence, des recirculations en amont d'une roue mobile de compresseur ou de pompe; ou encore localiser les ondes de choc à la périphérie d'un rotor de compresseur transsonique (fig. 3).
Tableau III

\begin{tabular}{|lc|}
\hline \multicolumn{2}{|c|}{ Caractéristiques d'un capteur $20 \mathrm{H} 48$} \\
\hline Étendue de mesure & $1 \mathrm{mbar}-10$ bars \\
Surcharge admissible & 10 fois l'entendue \\
& de mesure \\
Température maximale & $\leqslant 150^{\circ} \mathrm{C}$ \\
Impédance & $10^{11} \Omega$ \\
Capacité (pastille) & $39 \mathrm{pf}$ \\
Capacité (capteur) & $100 \mathrm{pf}$ \\
Sensibilité (avec adaptateur d'im- & $0,25 \mathrm{mV} / \mathrm{mbar}$ \\
pédance) & $2,8 \mathrm{pC} / \mathrm{bar}$ \\
Sensibilité (avec ampli de charge) & $<0,4 \mu \mathrm{s}$ \\
Temps de réponse & $\sim 600 \mathrm{khz}$ \\
Fréquence propre & $\sim 400 \mathrm{khz}$ \\
Bande passante de la chaine de & \\
mesure &
\end{tabular}

Ils peuvent également être montés en aval de l'orifice de prise de pression d'une sonde de type pitot ou cylindrique, de façon à obtenir des sondes de pressions instationnaires dont nous parlerons plus loin.

L'emploi de ces capteurs est bien adapté aux mesures industrielles où l'on se trouve souvent en présence de poussières, huile, etc.

\subsection{Capteurs piézorésistifs [2]}

Ce sont des capteurs à jauges intégrées montées en pont de Wheastone et diffusées dans un diaphragme en silicium (fig. 4).

Ils permettent de mesurer les pressions moyennes et fluctuantes dans un large domaine avec une excellente précision et dans une gamme de fréquence assez élevée.

Les plus connus sont certainement les capteurs " Kulite " qui, à leurs débuts, ont eu quelques problèmes au point de vue dérive de zéro et sensibilité à la température.

Toutefois, grâce à des efforts conjugués du constructeur et de certains utilisateurs, des améliorations très importantes ont pu être obtenues. A présent, ces capteurs ont une bonne résistance mécanique et ils fonctionnent très correctement jusque vers $235 \mathrm{C}$ (série haute température LE), en l'absence de tout dispositif de refroidissement. La dérive de zéro, la sensibilité à la température, ainsi que l'évolution dans le temps de la structure, ont été pratiquement éliminées.

D'autre part, un important effort de miniaturisation a été également entrepris et actuellement, il existe des capteurs dont l'épaisseur n'est que de $0,8 \mathrm{~mm}$, comme le montre à titre d'exemple la figure 5 (p. suiv.).

Le tableau $I V$ ci-dessous regroupe à titre indicatif les principales caractéristiques d'un tel capteur :

Tableau IV

\begin{tabular}{|ll|}
\hline \multicolumn{2}{|c|}{ Caractéristiques d'un capteur LQ 125} \\
\hline Gamme de mesure & de 5 à $500 \mathrm{psi}$ \\
Surcharge admissible & de 4 à 2 fois l'éten- \\
& due de mesure \\
Température d'utilisation & $-20^{\circ} \mathrm{C}$ à $120^{\circ} \mathrm{C}$ \\
Compensation en température & $25^{\circ} \mathrm{C}$ à $80^{\circ} \mathrm{C}$ \\
Niveau de sortie pleine échelle & 50 à $100 \mathrm{mV}$ \\
Fréquence propre & 70 à $350 \mathrm{khz}$ \\
Répétabilité & $0,25 \%$ \\
Non linéarité et hystérésis & $\pm 0,5 \%$ de l'éten- \\
& due de mesure \\
\hline
\end{tabular}




\begin{tabular}{|c|c|c|c|c|c|c|c|}
\hline SPECIFICATIONS & $\begin{array}{l}\text { Lo-125-5 } \\
\text { La-30-125-5 }\end{array}$ & $\begin{array}{l}\text { La } \\
\text { La-125-10 } \\
\end{array}$ & $\begin{array}{l}\text { LQ-125-25 } \\
\text { Lo-30-125-25 }\end{array}$ & $\begin{array}{l}\text { LO. } 125.50 \\
\text { LO-30.125.50 }\end{array}$ & $\begin{array}{l}\operatorname{L}(0.125 \cdot 100 \\
\operatorname{La}-30-125 \cdot 100\end{array}$ & $\begin{array}{l}\text { LO-125-200 } \\
\text { LO-30.125.200 }\end{array}$ & $\begin{array}{l}\text { L0.125.500 } \\
\text { LQ.30.125.500 }\end{array}$ \\
\hline Rated Pressure & 5 psi & $10 \mathrm{pmi}$ & $25 \mathrm{pq}$ & so do 1 & 100 Dsi & $200 \mathrm{psi}$ & 500 DSi \\
\hline Max. Pressure & $20 \mathrm{psi}$ & $20 \mathrm{pgi}$ & 100 pai & $100 \mathrm{psi}$ & 200 pai & $400 \mathrm{pr}$ & 1000 Dsi \\
\hline $\begin{array}{l}\text { Nominal Outout } \\
\text { at Aated Pressure }\end{array}$ & $50 \mathrm{mv}$ & $100 \mathrm{mV}$ & os $m v$ & $75 \mathrm{mV}$ & $100 \mathrm{mV}$ & $100 \mathrm{mv}$ & $100 \mathrm{mv}$ \\
\hline 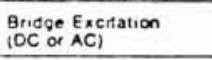 & $20 \mathrm{~V}$ & $20 \mathrm{v}$ & 10v & $10 \mathrm{~V}$ & 100 & $10 \mathrm{~V}$ & 10v \\
\hline $\begin{array}{l}\text { Inout Impedance } \\
\text { Outout Impecance }\end{array}$ & \multicolumn{2}{|c|}{$\begin{array}{l}4000 \text { onme (min.) } \\
3000 \text { onms (max.) }\end{array}$} & {$\left[\begin{array}{l}1500 \text { ohms (min.) } \\
1200 \text { onms (max.) }\end{array}\right]$} & \multicolumn{4}{|c|}{$\begin{array}{l}\infty 00 \text { onms (min) } \\
500 \text { onms (max) }\end{array}$} \\
\hline Zero Baiance & $=5 \times \mathrm{FS}$ & \multicolumn{6}{|c|}{$\Rightarrow \mathrm{XFS}$} \\
\hline $\begin{array}{l}\text { Combined } \\
\text { Non-inearty } 8 \\
\text { Hysteresie }\end{array}$ & \multicolumn{7}{|c|}{$=0.5 \times F S(\max )$} \\
\hline Repe atability & \multicolumn{7}{|c|}{0.256} \\
\hline $\begin{array}{l}\text { Comcensated } \\
\text { Temp. Aange }\end{array}$ & \multicolumn{7}{|c|}{ Any $100^{\circ} \mathrm{F}$ (55. C C iange within operating range on special arder } \\
\hline $\begin{array}{l}\text { Oporating } \\
\text { Temp. Aange }\end{array}$ & \multicolumn{7}{|c|}{ 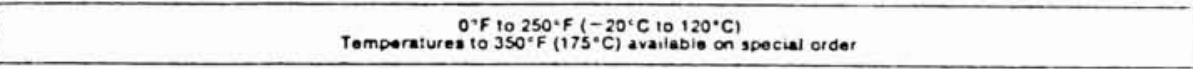 } \\
\hline $\begin{array}{l}\text { Change of Sonsitinty } \\
\text { with Temp. }\end{array}$ & \multicolumn{7}{|c|}{$\Rightarrow 2.5 \% / 100^{\circ} \mathrm{F}\left(55^{\circ} \mathrm{C}\right)$} \\
\hline $\begin{array}{l}\text { Change of Nat ond } \\
\text { Oulput with Tomp. }\end{array}$ & $\begin{array}{c}=3 \times F 5 / 1000 \\
\left(55^{\circ} \mathrm{C}\right)\end{array}$ & 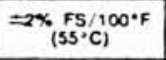 & \multicolumn{5}{|c|}{$\Rightarrow 1 \times \mathrm{FS} / 100^{\circ} \mathrm{F}\left(55^{\circ} \mathrm{C}\right)$} \\
\hline $\begin{array}{l}\text { Natural frequency } \\
\text { (approx.) }\end{array}$ & $70 \mathrm{KHz}$ & $70 \mathrm{KHz}$ & $100 \mathrm{KHz}$ & $130 \mathrm{KHz}$ & $180 \mathrm{KHz}$ & $200 \mathrm{KHz}$ & $350 \mathrm{KHz}$ \\
\hline $\begin{array}{l}\text { Aecoieration Soens. } \\
\text { Perpendiculat } \\
\text { Tranoverso }\end{array}$ & $\begin{array}{l}0.002 \% \text { FS: } \\
0.0004 \% \mathrm{FS}: 0\end{array}$ & $\begin{array}{l}0.001 \% \mathrm{FS} / 0 \\
0.0002 \% \mathrm{FS} ; 0\end{array}$ & $\begin{array}{l}0.0005 \%: F S: 0 \\
0.0001 \% \mathrm{FS} / 0\end{array}$ & $\begin{array}{rl}0.0004 \times & \mathrm{FS} \\
0.00008 \% & \mathrm{FS}\end{array}$ & $\begin{array}{l}0.0002 \% \text { FS : } \\
0.00004 \% \text { FS: }\end{array}$ & $\begin{array}{l}0.0002 \%: F S: ~ \\
0.00004 \% F S: G\end{array}$ & $\begin{array}{l}0.00000 \%: F S \\
0000016 \% \text { FS }\end{array}$ \\
\hline Resolvition & \multicolumn{7}{|c|}{ Intinte. } \\
\hline
\end{tabular}

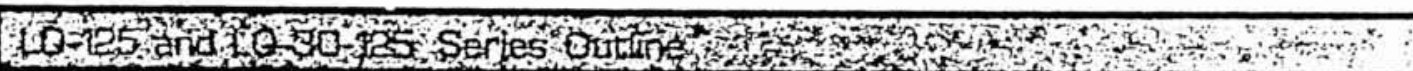

LQ-१२५

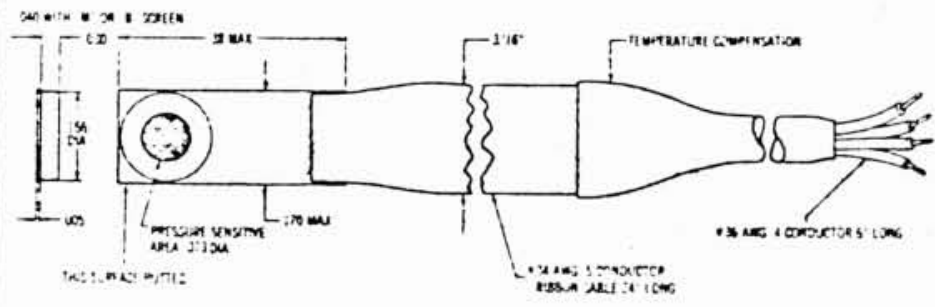

LQ-30-१२५

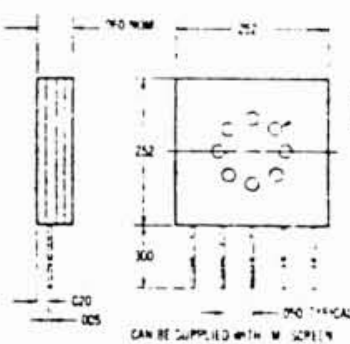

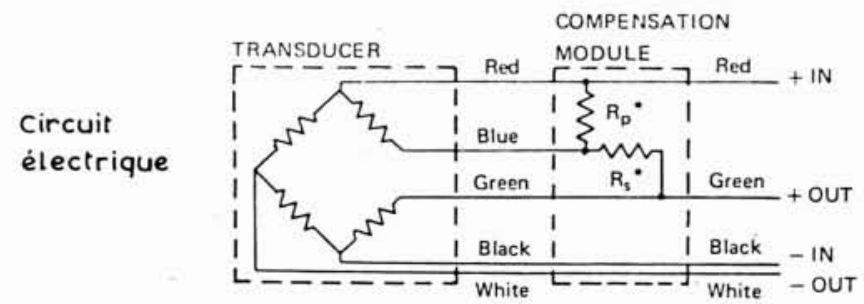

5. Capteurs Kulite miniatures et spécifications. 
De tels capteurs sont également très intéressants pour déterminer des champs de pression instationnaire au niveau d'une paroi. Mais leur petite taille et principalement leur faible épaisseur les rend particulièrement bien adaptés pour des mesures sur des parois minces, telles que des aubes de compresseur ou de ventilateur.

La figure 6 montre, par exemple, une aube mobile d'un compresseur, équipée, à $80 \%$ d'envergure, de ces capteurs miniatures. Les connexions électriques sont faites de circuits imprimés très minces $(0,08 \mathrm{~mm})$, déposés sur l'aube et les signaux sont transmis par un collecteur tournant.

Des capteurs piézorésistifs, plus classiques, peuvent également être utilisés pour réaliser des sondes de pression instationnaires. Pour cela, il existe des capteurs dont le diamètre n'est que de $0,8 \mathrm{~mm}$.

\subsection{Capteurs pelliculaires :}

Ce capteur, développé à l'ONERA au cours de ces dernières années [3], [4] est un détecteur capacitif constitué d'un diélectrique métallisé sur ses deux faces (fig. 7). Sous l'action d'une variation de pression $\Delta p$, l'épaisseur $e$ du diélectrique diminue de $\Delta e$. A cette variation d'épaisseur, correspond une variation de capacité $\Delta C$ qui s'exprime par : $(\Delta C / C)=-(\Delta e / e)$,

d'où la relation : $\Delta C / C=\Delta p / K$,

qui exprime que la variation relative de la capacité est proportionnelle à la variation de la pression appliquée, $(K$ étant le module de compression uniaxial).

Quant à la chaine de mesure associée, la solution choisie est celle de la polarisation continue du condensateur, avec transmission du signal par adaptateur d'impédance de façon à réduire au minimum les perturbations du signal par le rayonnement électromagnétique. Cet adaptateur d'impédance est réalisé en électronique hybride et peut être implanté à proximité de l'élément sensible.

De tels capteurs ont été développés en vue de leur application aux études de turbomachines et devaient répondre aux conditions suivantes : être de petites dimensions et d'une épaisseur telle qu'ils puissent être implantés sur des parois, et principalement sur des aubes, sans usinage préalable des pièces et sans perturber le champ aérodynamique.

La possibilité de réaliser ces capteurs résulte de l'utilisation de diélectrique souple Kapton qui existe en faible épaisseur (6 à $100 \mu \mathrm{m}$ ), qui a un faible module élastique ( $E=30000$ bar) et qui a une bonne tenue en température $\left(\sim 300^{\circ} \mathrm{C}\right)$. Elle résulte également des possibilités offertes par l'évaporation sous vide ou la pulvérisation cathodique, pour réaliser les dépôts métalliques des éléments sensibles et les conducteurs acheminant le signal (couches minces de $2000 \AA$ ). Elle résulte enfin de la mise au point de techniques de collage à l'aide de films de colle de très faible épaisseur ( 2 à $4 \mu \mathrm{m}$ ).

Parallèlement à ces capteurs à diélectrique solide (Kapton), des capteurs à diélectrique gazeux ont été réalisés (fig. 8). Le principe consiste à réaliser un élément capacitif entre les électrodes duquel sont crées de multiples alvéoles remplies de gaz. Ces alvéoles sont formées

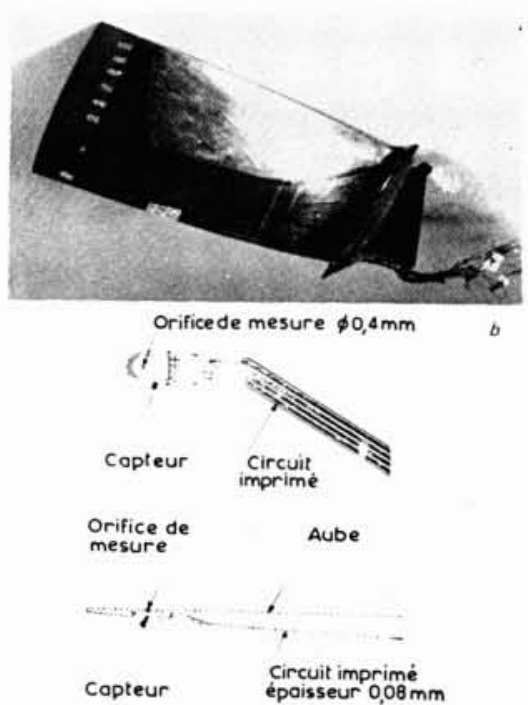

6. Implantation de capteurs miniatures sur une aube de compresseur.

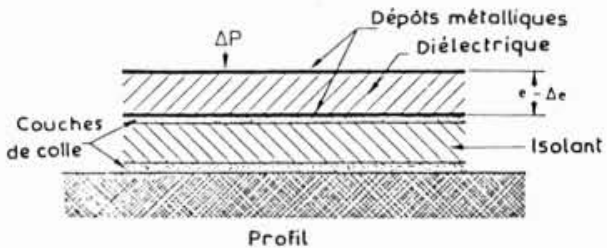

7. Principe du capteur pelliculaire de pression à diélectrique solide.
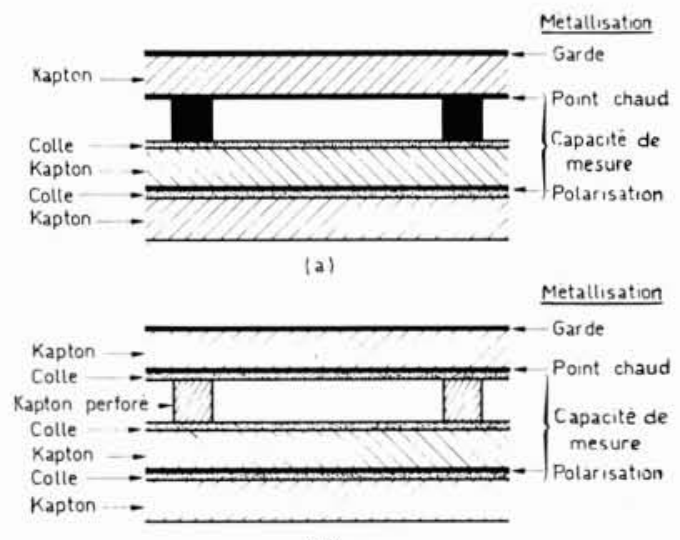

(b)

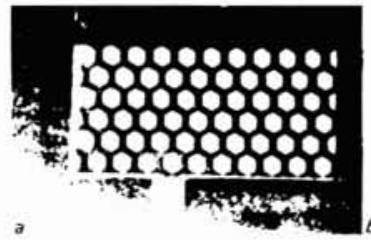

Murs métaltiques

Murs isolants

8. Principe du capteur pelliculaire de pression à diélectrique gazeux (murs métalliques ou murs isolants). 

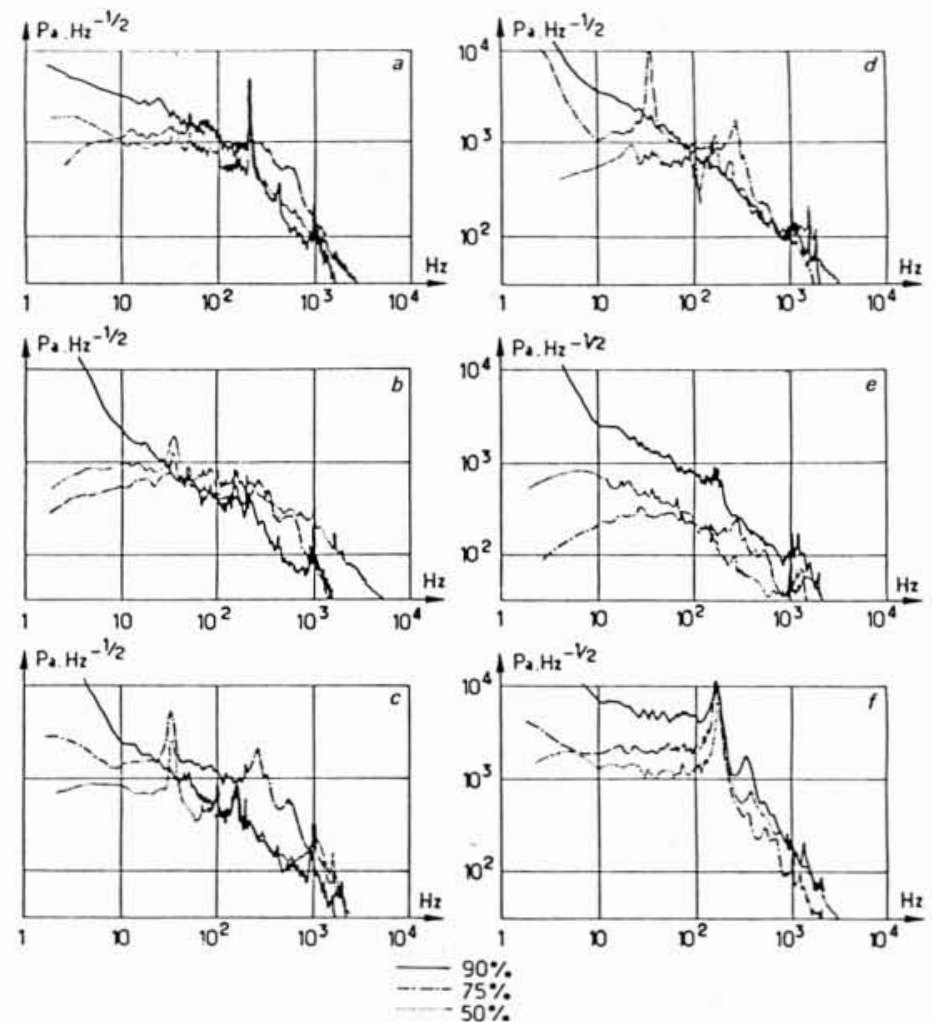

10. Aube de compresseur équipée de capteurs pelliculaires de pression.

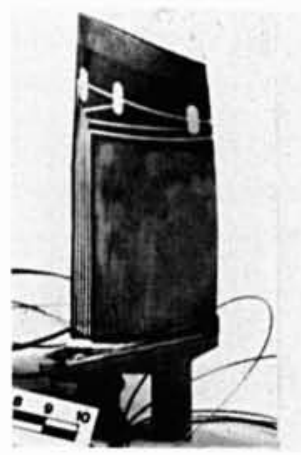

9. Spectres de fluctuations de pression à lextrados d'une aube montée en grille annulaire fixe supersonique. Influence de la contrepression.

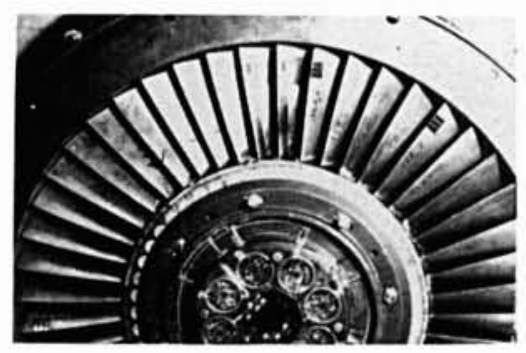

11. Vue du rotor - de !'électronique.
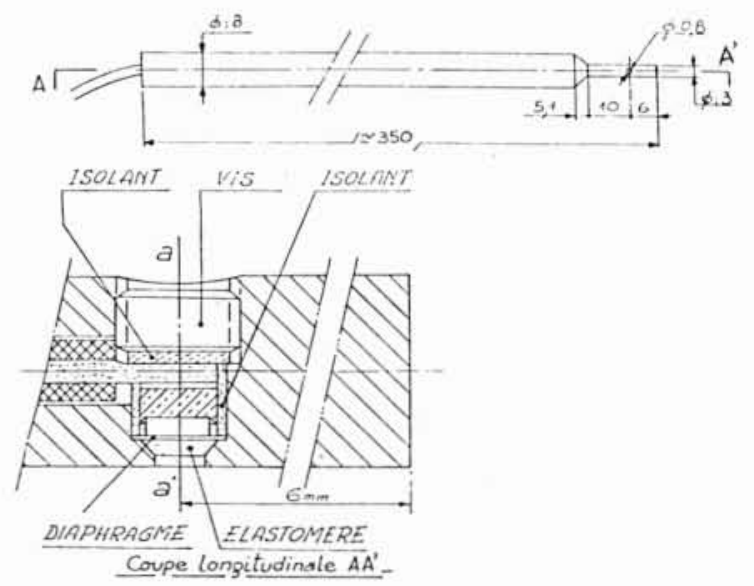

13. Sonde cylindrique de pression instationnaire à capteur affleurant. Diamètre $=3 \mathrm{~mm}$.

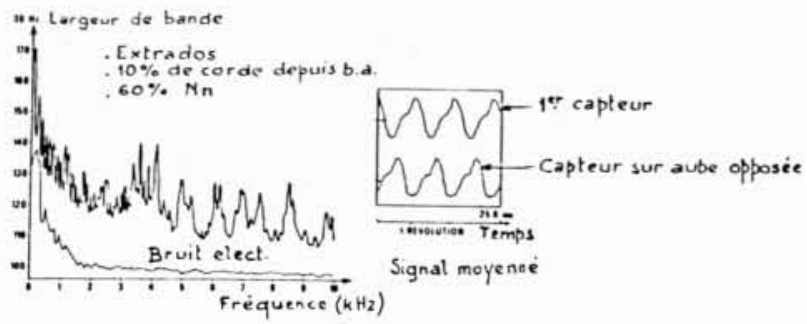

12. Spectre déduit des mesures de pression instationnaire. 
par des films diélectriques en Kapton, le film supérieur étant utilisé comme membrane déformable. Les séparations entre alvéoles (murs), peuvent être métalliques ou isolantes.

En ce qui concerne la sensibilité à la pression de ces capteurs pelliculaires, elle reste constante en fonction de la fréquence, depuis le continu jusqu'à plus de $100 \mathrm{kHz}$ pour les capteurs à diélectrique solide en milieu liquide. En milieu gazeux, la sensibilité de ce capteur, du fait des fluctuations de température induites par les fluctuations de pression, évolue en fonction de la fréquence pour atteindre au-dessus de $10 \mathrm{kHz}$ une sensibilité due au seul écrasement. Une correction est possible pour les mesures de pression instantanée en basse fréquence $(<10 \mathrm{kHz})$.

Quant aux capteurs à diélectrique gazeux, leur sensibilité à la pression reste indépendante de la fréquence.

Les principales caractéristiques de ces capteurs pelliculaires sont résumées dans le tableau $V$ :

Tableau V

\begin{tabular}{|c|c|c|}
\hline \multicolumn{3}{|c|}{ Principales caractéristiques des capteurs pelliculaires } \\
\hline & $\begin{array}{l}\text { Capteurs à } \\
\text { diélectrique } \\
\text { solide }\end{array}$ & $\begin{array}{c}\text { Capteurs à } \\
\text { diélectrique } \\
\text { gazeux } \\
\text { (Membranes } \\
\text { épaisseur } \\
12 \mu \mathrm{m} \text { ) }\end{array}$ \\
\hline & $\begin{array}{l}\text { variable avec } \\
\text { la fréquence }\end{array}$ & \\
\hline Sensibilité $(\Delta C / C$ par pascal) & $2.10^{-9}$ à $2,5.10^{-10}$ & $3.10^{-7}$ \\
\hline Bande passante $(\mathrm{Hz})$ & 1 à $10^{5}$ & 1 à $10^{5}$ \\
\hline $\begin{array}{l}\text { Détectivité à } 1 \mathrm{kHz}(\mathrm{Pa} / \sqrt{ } \mathrm{Hz}) \\
\text { (polarisé sous } 100 \mathrm{~V} \text { ) }\end{array}$ & 2 & $10^{-1}$ \\
\hline Epaisseur du capteur ( $\mu \mathrm{m})$ & 50 & 80 \\
\hline Température d'utilisation $\left({ }^{\circ} \mathrm{C}\right)$ & 0 à 60 & 0 à 60 \\
\hline
\end{tabular}

Quant au domaine d'application de ces capteurs, il est très vaste dans les gaz comme dans les liquides, que ce soit pour mesurer des pressions instantanées ou des spectres de fluctuations.

A titre d'exemple, la figure 9 montre les spectres de fluctuations de pression obtenues à l'extrados d'une aube montée en soufflerie de grille d'aubes fixe supersonique [5]. Les signaux se rapportent à trois capteurs implantés à mi-envergure et pour diverses valeurs de la contrepression aval depuis un régime amorcé jusqu'à un fonctionnement désamorcé.

Un autre exemple d'application concerne des mesures sur roue mobile d'un compresseur aéronautique [6]. La figure 10 montre une aube équipée et la figure 11 , une vue du rotor avec l'électronique associée. Un spectre déduit des mesures de pression instationnaire est indiqué sur la figure 12, et correspond à un capteur placé à l'extrados près du bord d'attaque. La figure montre également que le rapport signal sur bruit est toujours supérieur à $10 \mathrm{~dB}$, tandis que sur la partie droite, le signal moyenné du capteur est comparé à celui d'un autre capteur situé sur une aube diamétralement opposée. On observe que les deux signaux ont des allures très semblables, mais sont déphasés en fonction de l'écart angulaire des deux aubes concernées.

\section{Mesures de vitesse}

La détermination des vitesses s'effectue généralement à partir de mesures de pression. De ce fait, le développement des capteurs de pression, dont nous venons de parler, a permis des progrès dans la mesure des vitesses et principalement des vitesses instantanées. La technique du fil chaud a également progressé, mais elle a certaines limitations d'emploi dans les liquides.

D'autre part l'utilisation de sondes apporte des perturbations dont l'importance est souvent loin d'être négligeable et ne permet que difficilement d'effectuer des mesures dans des parties mobiles (sondes tournantes); d'oủ l'intérêt des méthodes récentes basées sur l'optique et l'acoustique. De même, le développement des dispositifs de traitement numérique du signal a permis d'obtenir un outil puissant de mesure des vitesses.

Le tableau $V I$ répertorie ces diverses méthodes :

Tableau VI

\begin{tabular}{|ll|}
\hline \multicolumn{2}{|c|}{ Moyens pour la détermination des vitesses } \\
\hline $3-1$ & Sondes de pression instationnaires \\
$3-2$ & Fils et films chauds \\
$3-3$ & Vélocimètre laser \\
$3-4$ & Corrélation \\
$3-5$ & Analyse d'image \\
$3-6$ & Sodar \\
\hline
\end{tabular}

\subsection{Sondes de pressions instationnaires}

La miniaturisation de capteurs de pression instationnaire a permis la réalisation de sondes diverses, bien adaptées à la mesure des vitesses instantanées à la sortie d'une roue mobile [7]. Moyennant un circuit de refroidissement approprié, de telles sondes peuvent être utilisées jusqu'à des températures de plusieurs centaines de degrés.

A partir d'un capteur piézoélectrique du type de celui décrit précédemment, une sonde cylindrique a été réalisée, comme le montre la figure 13, en vue d'explorer l'écoulement à la sortie d'une roue mobile d'un compresseur.

La procédure d'emploi consiste à relever les répartitions de pression sur la sonde correspondant à une position du rotor bien définie au cours de chaque cycle. Pour cela, les enregistrements de la pression, en fonction du temps, (échantillonnage par moyenneur), sont répétées pour plusieurs valeurs discrètes de l'angle d'orientation $\theta$ de la sonde.

Un programme sur ordinateur rassemble automatiquement les valeurs expérimentales $p(\theta)$ obtenues pour une même valeur constante de l'abscisse temporelle relative $t / T$, puis les ajuste, par une méthode des moindres carrés, sur une courbe du réseau d'étalonnage de la sonde $p / P=f(\theta, M)$ obtenu en soufflerie.

Cette opération permet de déduire la pression d'arrêt $P$. l'orientatin de la vitesse, ainsi que cette dernière à l'instant $t / T$ considéré. Elle est répétée pour 100 valeurs équiréparties dans la période $T$. Les résultats obtenus ainsi dans le référentiel lié à la sonde sont en outre transférés dans un repère mobile lié au rotor. 
Tableau VII

\begin{tabular}{|c|c|c|c|c|c|c|c|c|c|c|c|c|c|}
\hline $\begin{array}{l}\text { SENSOR } \\
\text { TYPE }\end{array}$ & 总旡 & 产 & 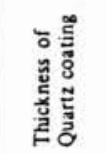 & 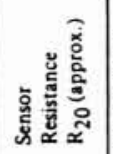 & 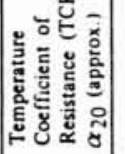 & 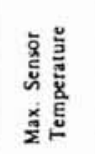 & 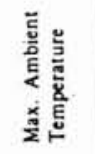 & 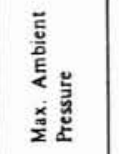 & 竞紊 & $\frac{\stackrel{2}{\bar{H}}}{\frac{\pi}{2}}$ & 离 & 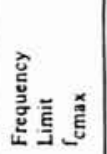 & $\frac{E}{\frac{E}{\tilde{z}}}$ \\
\hline \multirow{2}{*}{$\begin{array}{l}\text { Gold-plated } \\
\text { Wire Sensors }\end{array}$} & \multirow{2}{*}{$\begin{array}{l}\text { Platinum- } \\
\text { plated } \\
\text { tungsten }\end{array}$} & \multirow{2}{*}{\begin{tabular}{|l|}
$5 \mu \mathrm{m} \mathrm{dia}$ \\
$1.25 \mathrm{~mm}$ \\
long $\quad$ " \\
\end{tabular}} & \multirow{2}{*}{ - } & \multirow{2}{*}{$3.5 \Omega$} & \multirow{2}{*}{$0.36 \% /{ }^{\circ} \mathrm{C}$} & \multirow{2}{*}{$300^{\circ} \mathrm{C}$} & \multirow{2}{*}{$150^{\circ} \mathrm{C}$} & \multirow{2}{*}{$-{ }^{21}$} & $0.2 \mathrm{~m} / \mathrm{s}$ & $200 \mathrm{~m} / \mathrm{s}$ & $90 \mathrm{~Hz}$ & $400 \mathrm{kHz}$ & air \\
\hline & & & & & & & & & $0.01 \mathrm{~m} / \mathrm{s}$ & - & - & - & dist. water \\
\hline \multirow{2}{*}{$\begin{array}{l}\text { Miniature } \\
\text { Wire Sensors }\end{array}$} & \multirow{2}{*}{$\begin{array}{l}\text { Platinum- } \\
\text { plated } \\
\text { tungsten }\end{array}$} & \multirow{2}{*}{\begin{tabular}{|l|}
$5 \mu \mathrm{m} \mathrm{dia}$ \\
$1.25 \mathrm{~mm}$ \\
long
\end{tabular}} & \multirow{2}{*}{ - } & \multirow{2}{*}{$3.5 \Omega$} & \multirow{2}{*}{$0.36 \% /^{\circ} \mathrm{C}$} & \multirow{2}{*}{$300^{\circ} \mathrm{C}$} & \multirow{2}{*}{$150^{\circ} \mathrm{C}$} & \multirow{2}{*}{$-{ }^{21}$} & $0.2 \mathrm{~m} / \mathrm{s}$ & $500 \mathrm{~m} / \mathrm{s}$ & $90 \mathrm{~Hz}$ & $400 \mathrm{kHz}$ & air \\
\hline & & & & & & & & & $0.01 \mathrm{~m} / \mathrm{s}$ & $5 \mathrm{~m} / \mathrm{s}$ & - & - & dist water \\
\hline \multirow{2}{*}{$\begin{array}{l}\text { Subminiature } \\
\text { Wire Sensors }\end{array}$} & \multirow{2}{*}{$\begin{array}{l}\text { Platinum- } \\
\text { plated } \\
\text { tungsten }\end{array}$} & \multirow{2}{*}{$\begin{array}{l}5 \mu \mathrm{m} \mathrm{dia} \\
0.45 \mathrm{~mm} \\
\text { long } \\
\end{array}$} & \multirow{2}{*}{-} & \multirow{2}{*}{$1.3 \Omega$} & \multirow{2}{*}{$0.36 \% /{ }^{\circ} \mathrm{C}$} & \multirow{2}{*}{$300^{\circ} \mathrm{C}$} & \multirow{2}{*}{$150^{\circ} \mathrm{C}$} & \multirow{2}{*}{$-{ }^{22}$} & $0.2 \mathrm{~m} / \mathrm{s}$ & $500 \mathrm{~m} / \mathrm{s}$ & $300 \mathrm{~Hz}$ & $150 \mathrm{kHz}$ & air \\
\hline & & & & & & & & & $0.01 \mathrm{~m} / \mathrm{s}$ & $5 \mathrm{~m} / \mathrm{s}$ & - & - & dist.water \\
\hline $\begin{array}{l}\text { Wire Sensors } \\
\text { for High } \\
\text { Temperatures } \\
\end{array}$ & $\begin{array}{l}\text { Platinum/ } \\
\text { Rhodium } \\
(90 / 10)\end{array}$ & \begin{tabular}{|l|}
$10 \mu \mathrm{m}$ dia \\
$2.2 \mathrm{~mm}$ \\
long \\
\end{tabular} & - & $6 \Omega$ & $0.16 \% /{ }^{\circ} \mathrm{C}$ & $800^{\circ} \mathrm{C}$ & $750^{\circ} \mathrm{C}$ & 100 bar & $0.2 \mathrm{~m} / \mathrm{s}$ & $350 \mathrm{~m} / \mathrm{s}$ & $25 \mathrm{~Hz}$ & $150 \mathrm{kHz}$ & air \\
\hline $\begin{array}{l}\text { Wire Sensors } \\
\text { for Temperature } \\
\text { Measurements } 3\end{array}$ & Platinum & \begin{tabular}{|l|}
$1 \mu \mathrm{m} \mathrm{dia}$ \\
$0.4 \mathrm{~mm}$ \\
long \\
\end{tabular} & - & $50 \Omega$ & $0.35 \% \%^{\circ} \mathrm{C}$ & - & $150^{\circ} \mathrm{C}$ & $-\quad 21$ & - & $60 \mathrm{~m} / \mathrm{s}$ & $2 \mathrm{kHz}$ & - & air \\
\hline \multirow{2}{*}{\begin{tabular}{l|} 
Fiber-Film \\
Sensors
\end{tabular}} & \multirow{2}{*}{ Nickel } & \multirow{2}{*}{\begin{tabular}{|l|}
$70 \mu \mathrm{m}$ dia \\
$1.25 \mathrm{~mm}$ \\
long
\end{tabular}} & $0.5 \mu \mathrm{m}$ & $6 \Omega$ & $0.40 \% /{ }^{\circ} \mathrm{C}$ & $300^{\circ} \mathrm{C}$ & $150^{\circ} \mathrm{C}$ & - & $0.2 \mathrm{~m} / \mathrm{s}$ & $350 \mathrm{~m} / \mathrm{s}$ & - & $175 \mathrm{kHz}$ & air \\
\hline & & & $2 \mu \mathrm{m}$ & $6 \Omega$ & $0.40 \% /{ }^{\circ} \mathrm{C}$ & $150^{\circ} \mathrm{C}$ & $100^{\circ} \mathrm{C}$ & - & $0.01 \mathrm{~m} / \mathrm{s}$ & $10 \mathrm{~m} / \mathrm{s}$ & - & $30 \mathrm{kHz}$ & water \\
\hline $\begin{array}{l}\text { Split-Fiber } \\
\text { Sensors }\end{array}$ & Nickel & \begin{tabular}{|l|}
$200 \mu \mathrm{m} \mathrm{dia}$ \\
$1.25 \mathrm{~mm}$ \\
long \\
\end{tabular} & $0.5 \mu \mathrm{m}$ & $6 \Omega$ & $0.4 \% /{ }^{\circ} \mathrm{C}$ & $300^{\circ} \mathrm{C}$ & $150^{\circ} \mathrm{C}$ & - & $0.2 \mathrm{~m} / \mathrm{s}$ & $350 \mathrm{~m} / \mathrm{s}$ & - & $175 \mathrm{kH} /$ & air \\
\hline Wedge-shaped & Nickel & $1 \mathrm{~mm} x$ & $0.5 \mu \mathrm{m}$ & $15 \Omega$ & $0.30 \% 1^{\circ} \mathrm{C}$ & $300^{\circ} \mathrm{C}$ & $150^{\circ} \mathrm{C}$ & 70 bar & $0.1 \mathrm{~m} / \mathrm{s}$ & $500 \mathrm{~m} / \mathrm{s}$ & $0.05 \mathrm{~Hz}$ & $450 \mathrm{kHz}$ & air \\
\hline & & $0.2 \mathrm{~mm}$ & $2 \mu \mathrm{m}$ & $15 \Omega$ & $0.30 \% /{ }^{\circ} \mathrm{C}$ & $150^{\circ} \mathrm{C}$ & $100^{\circ} \mathrm{C}$ & 70 bar ${ }^{31}$ & $0.01 \mathrm{~m} / \mathrm{s}$ & $25 \mathrm{~m} / \mathrm{s}$ & $0.4 \mathrm{~Hz}$ & $30 \mathrm{kHz}$ & water \\
\hline Conical & Nickel & $1.4 \mathrm{~mm} x$ & $0.5 \mu \mathrm{m}$ & $15 \Omega$ & $0.35 \% /{ }^{\circ} \mathrm{C}$ & $300^{\circ} \mathrm{C}$ & $150^{\circ} \mathrm{C}$ & 70 bat & $0.1 \mathrm{~m} / \mathrm{s}$ & $500 \mathrm{~m} / \mathrm{s}$ & - & - & sir \\
\hline Film Sensors & Nicket & $0.1 \mathrm{~mm}$ & $2 \mu \mathrm{m}$ & $15 \Omega$ & $0.35 \% 1^{\circ} \mathrm{C}$ & $150^{\circ} \mathrm{C}$ & $100^{\circ} \mathrm{C}$ & 70 bar ${ }^{51}$ & $0.01 \mathrm{~m} / \mathrm{s}$ & $25 \mathrm{~m} / \mathrm{s}$ & - & $30 \mathrm{kHz}$ & water \\
\hline Flush-mountung & Nivel & $0.75 \mathrm{~mm}$ & $0.5 \mu \mathrm{m}$ & $15 \Omega$ & $0.35 \% /{ }^{\circ} \mathrm{C}$ & $200^{\circ} \mathrm{C}$ & $120^{\circ} \mathrm{C}$ & $70 \mathrm{bar}$ & - & - & $0.2 \mathrm{~Hz}$ & - & atr \\
\hline Film Senwors & NILEet & $\times 0.2 \mathrm{~mm}$ & $24 \mathrm{~m}$ & $15 \Omega$ & $0.35 \% 7^{\circ} \mathrm{C}$ & $150^{\circ} \mathrm{C}$ & $100^{\circ} \mathrm{C}$ & 70 bar ${ }^{51}$ & - & - & $0.5 \mathrm{~Hz}$ & $30 \mathrm{kHz}$ & water \\
\hline Glue-un & Nickel & $1 \mathrm{~mm} x$ & $0.5 \mu \mathrm{m}$ & $15 \Omega$ & $0.43 /{ }^{\circ} \mathrm{C}$ & $200^{\circ} \mathrm{C}$ & $120^{\circ} \mathrm{C}$ & - & - & - & - & - & ait \\
\hline Film Sensurs & Nickel & $0.2 \mathrm{~mm}$ & - & - & - & - & - & - & - & - & - & - & - \\
\hline $\begin{array}{l}\text { Ominidifectional } \\
\text { Probe }\end{array}$ & Nickel & $\begin{array}{l}2.6 \mathrm{~mm} \\
\text { sphere }\end{array}$ & $0.5 \mu \mathrm{m}$ & $25 \Omega$ & $056 / 0 \mathrm{C}$ & $\mathrm{OU}^{\circ} \mathrm{C} \mathrm{C}$ & $\angle n 0^{\circ} \mathrm{C}$ & - & $0.05 \mathrm{~m} / \mathrm{s}$ & $1 \mathrm{~m} / \mathrm{s}$ & $-0.2 \mathrm{~Hz}$ & $-x \mathrm{H}_{2}$ & sit \\
\hline Steel Clad & Nukel & $1.1 \mathrm{man} x$ & - & & $00=010$ & 1000 & $800^{\circ} \mathrm{C}$ & $70 \mathrm{bur} \mathrm{r}^{51}$ & $002 \mathrm{~m} / \mathrm{s}$ & $100 \mathrm{~m} / \mathrm{s}$ & $-0.411 t$ & -211, & sir \\
\hline Prohe & Nickel & $6 \mathrm{~mm}$ & 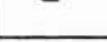 & $10 \Omega$ & C.? & $\mathrm{xo}^{\circ} \mathrm{C} \mathrm{C}$ & $(6)^{\circ 1} \mathrm{C}$ & $70 \mathrm{bur} 51$ & $0005 \mathrm{~m} / \mathrm{s}$ & $3 \mathrm{~m} / \mathrm{s}$ & - & - & water \\
\hline NOTES & & 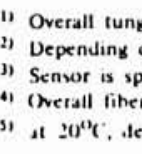 & $\begin{array}{l}\text { ecially sui } \\
\text { length is } \\
\text { cressng }\end{array}$ & 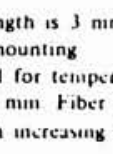 & 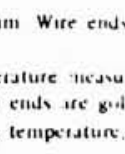 & 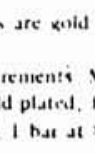 & 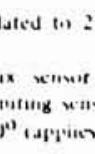 & $\begin{array}{l}-30 \mu \mathrm{m} \text { dis } \\
\text { uricout I m } \\
\text { of length } \\
\text { to probe des }\end{array}$ & $\begin{array}{l}\text { and limit - } \\
\text { sign listeds }\end{array}$ & enwir length & & & \\
\hline
\end{tabular}

La technique décrite repose sur l'hypothèse que le champ d'écoulement autour de la sonde à un instant donné, correspond à celui qui serait observé en écoulement uniforme et permanent de mêmes vitesse et orientation; il est donc nécessaire d'avoir une sonde la plus petite possible.

La figure 14 montre une comparaison de résultats obtenus avec une telle sonde et une sonde à fil chaud en ce qui concerne les vitesses et direction de l'écoulement en axes absolus et relatifs [8].

On observe une bonne concordance d'ensemble, mais la largeur du sillage donné par le fil chaud étant du même ordre de grandeur que le diamètre de la sonde, il en résulte une perturbation locale de ce sillage se traduisant par une épaisseur plus étendue et une amplitude réduite de cette zone lorsque les mesures sont effectuées avec la sonde de pression. Une sonde de pression plus petite aurait conduit à un meilleur accord.

A partir d'un capteur piézorésistif Kulite du type d'un de ceux décrits précédemment, une autre sonde de pression instationnaire [9] a été réalisée comme le montre la figure 15.

La face sensible du capteur de pression est directement placée face à l'écoulement. Toutefois, un rebord à lèvres minces entoure le capteur, disposition qui, en stationnaire, permet d'obtenir une pression uniforme sur la face du capteur pour une large plage d'angles d'attaque.

Cette sonde a été utilisée pour mesurer les variations périodiques de la pression d'arrêt, d'où la vitesse, à la sortie d'une roue mobile. Comme précédemment, la procédure expérimentale consiste à effectuer des mesures pour différentes positions angulaires $\theta$ de la sonde. On représente, en fonction de $\theta$, la pression mesurée à un instant donné de la période élémentaire. Le maximum de la courbe indique la pression d'arrêt locale à l'abscisse temporelle relative $t / T$ choisie. La direction locale de l'écoulement est également obtenue.

Le résultat final d'une telle exploration a été comparé 


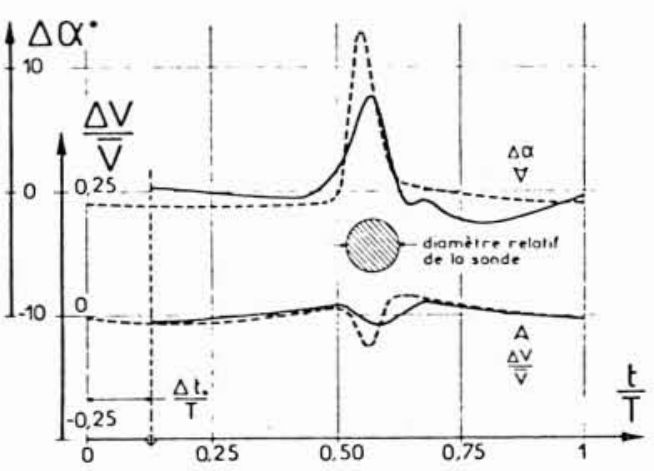

15. Sonde de pression instationnaire avec capteur piézorésistif.
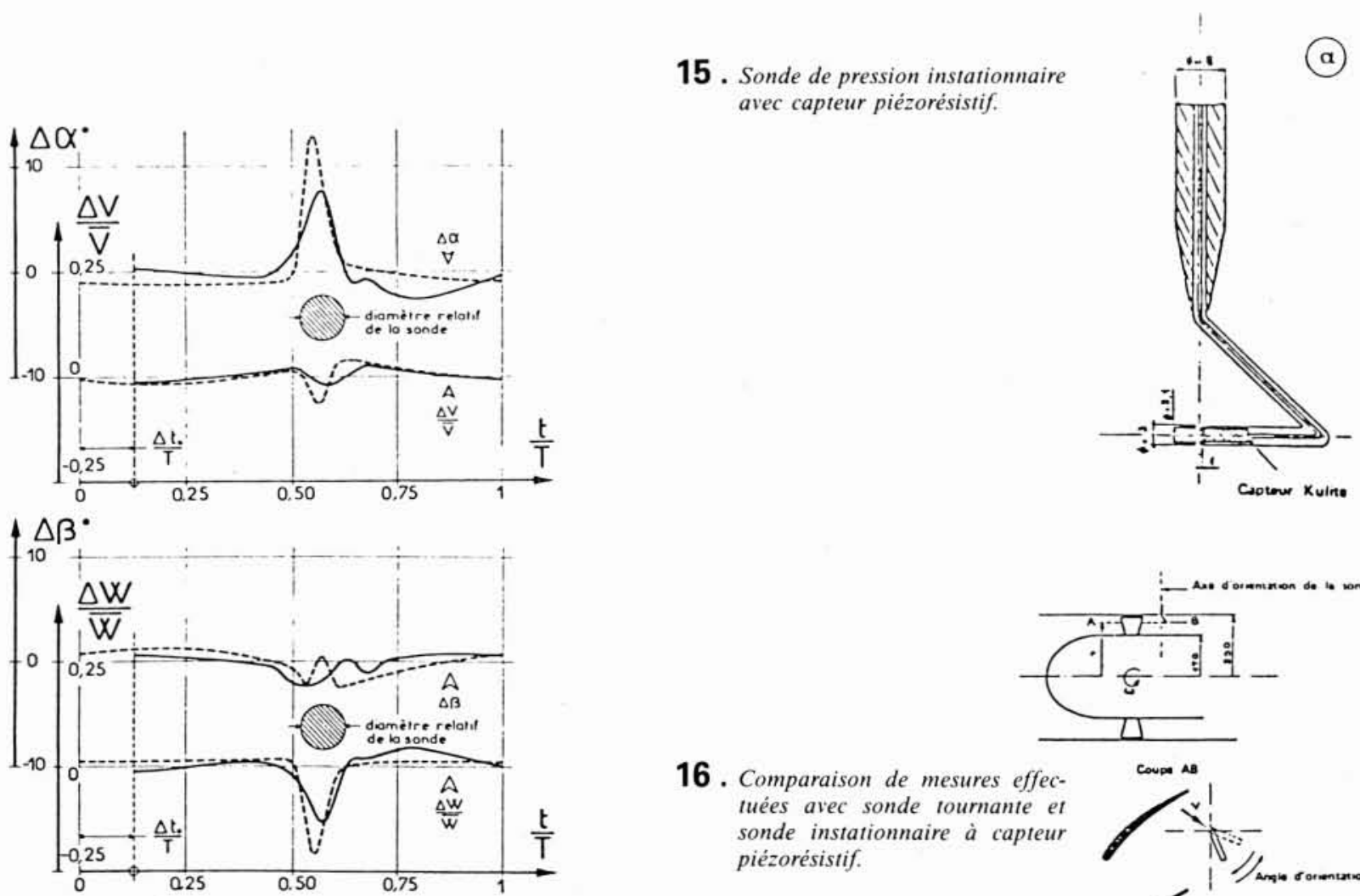

Sonde à fil chaud.

Sonde de pression.

Vitesse et direction de l'écoulement :

- mouvement absolu : $\mathbf{V}$ et $\alpha$;

- mouvement relatif au rotor: $\mathrm{W}$ et $\beta$;

$\stackrel{t}{\mathbf{T}}$ : Fraction d'un pas interaubes.

14. Comparaison de mesures effectuées avec sonde de pression instationnaire et sonde à fil chaud.

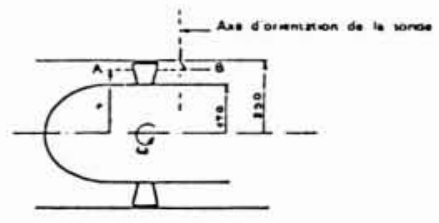

16. Comparaison de mesures effectuées avec sonde tournante et sonde instationnaire à capteur piézorésistif.
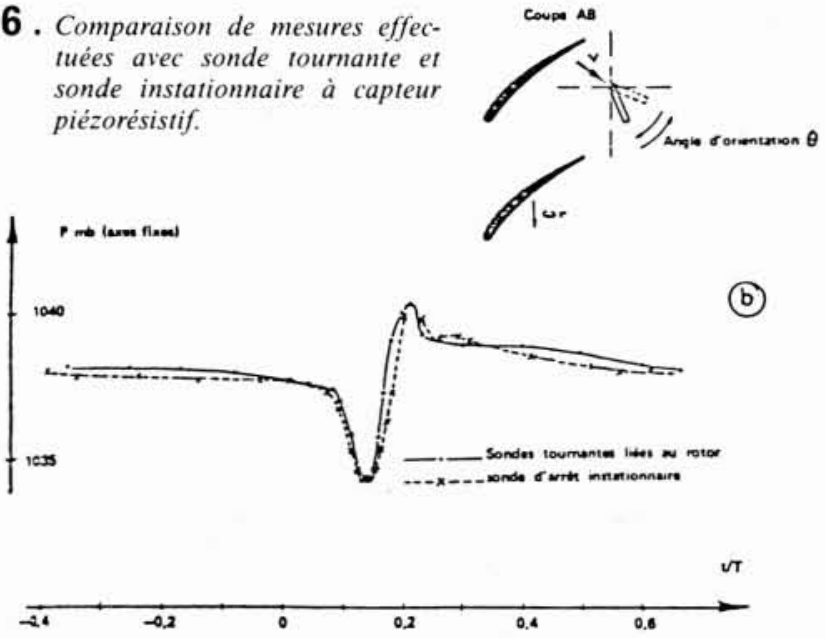

(b)

avec celui déduit de mesures effectuées à l'aide d'une sonde tournante, liée au rotor. Comme le montre la figure 16. l'accord est très satisfaisant.

Parallèlement à ces deux types de sondes à capteur unique, une sonde instationnaire à cinq trous a été réalisée. Les premiers résultats sont très encourageants mais, malgré la miniaturisation des capteurs, une telle sonde nécessite des veines d'essais importantes qui limitent, du moins actuellement, son domaine d'utilisation.

\subsection{Fils et films chauds}

Sans rentrer dans le détail du principe bien connu de l'anémométrie à fil chaud, nous rappelerons seulement que la technique de mesure la plus utilisée est l'anémométrie à température constante. La tension $V$ de déséquilibre du pont, dont le fil chaud est une branche, est reliée à la vitesse $U$ de l'écoulement par une relation du type

$$
V^{2}=A+B U^{n}
$$

$A$ et $B$ sont des constantes déterminées par étalonnage tandis que l'exposant $n$, voisin de 0,5 , peut varier avec le fluide et la plage de vitesse.

En ce qui concerne les fils chauds, les sondes standard Dantec permettent de mesurer des vitesses maximales dans l'air de $500 \mathrm{~m} / \mathrm{s}$ et dans l'eau distillée de $5 \mathrm{~m} / \mathrm{s}$.

Les films chauds ont une résistance mécanique plus élevée et sont utilisables dans les gaz jusqu'à des niveaux de vitesse importants et dans les liquides jusqu’à $25 \mathrm{~m} / \mathrm{s}$ environ.

Quant à la configuration des sondes, on rencontre couramment des sondes à un, deux ou trois éléments [10]. Chaque élément mesure une composante de la vitesse dans un plan qui lui est perpendiculaire. Ainsi, si les trois éléments sensibles sont orthogonaux, on peut obtenir une représentation spatiale de la vitesse. 


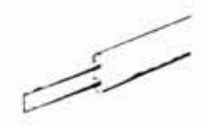

Sonde o if it

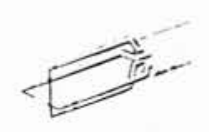

Sonde à 2 rils

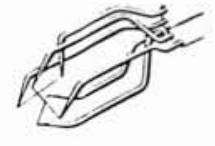

Sonde ò 3 fils
17. Exemples type de sondes à fil chaud.

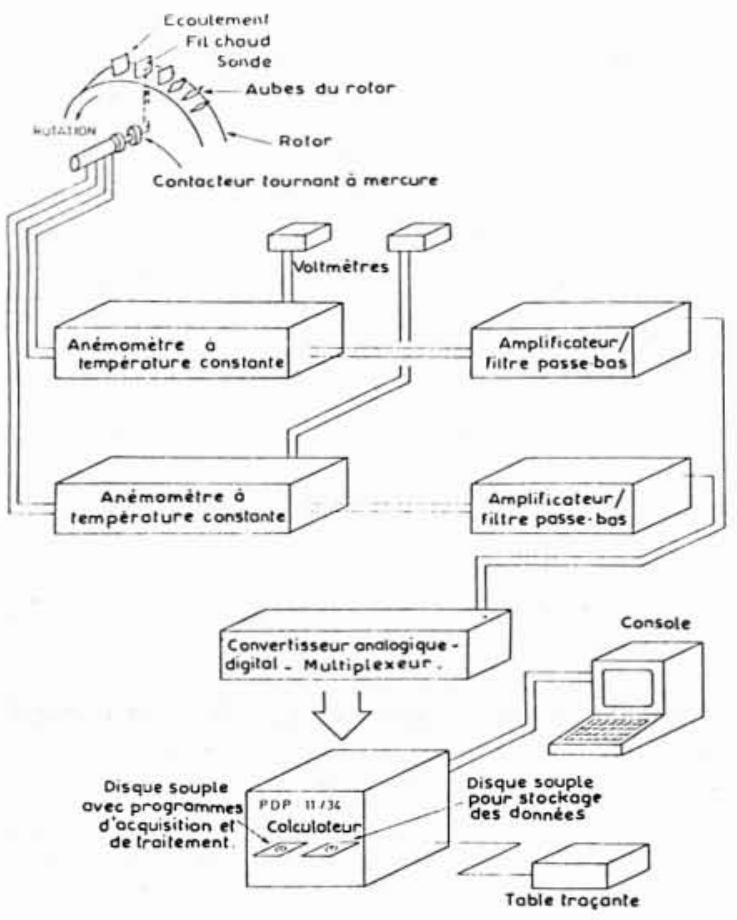

18. Schéma de la sonde spéciale à deux fils parallèles.

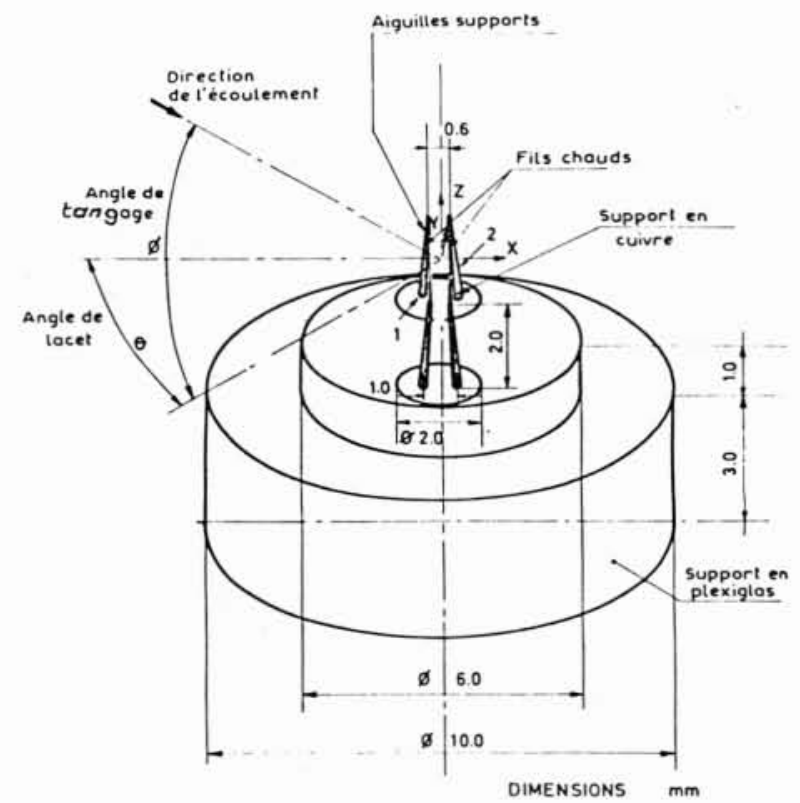

19. Schéma de la chaine de mesure.
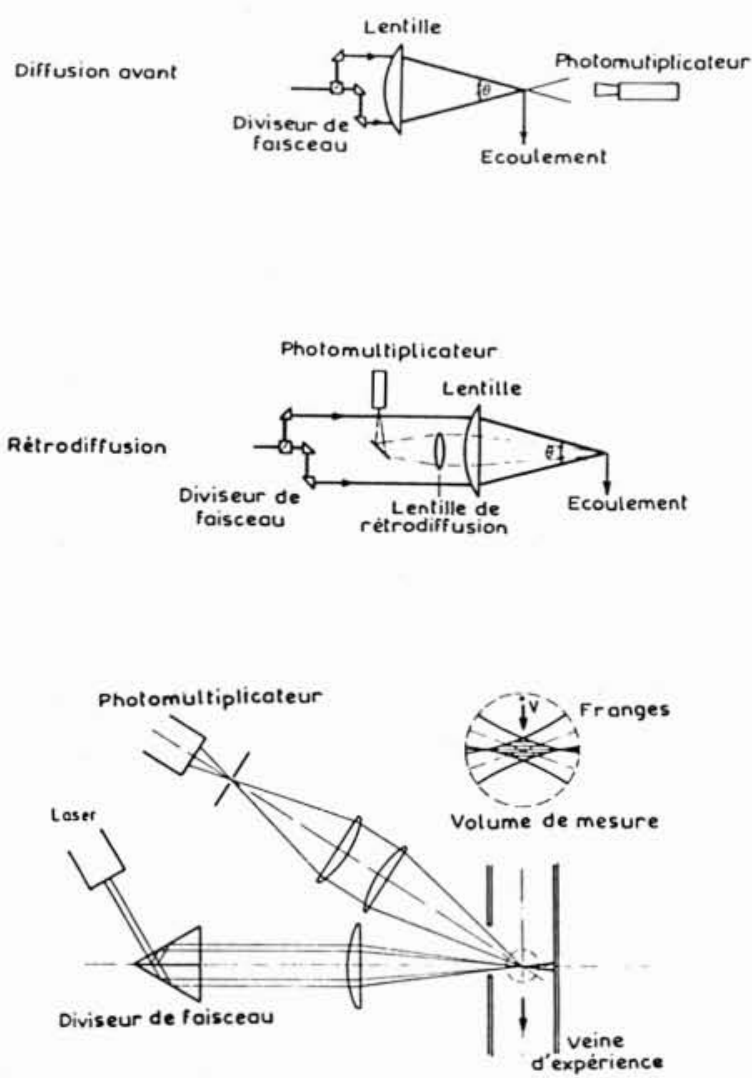

20. Anémométrie laser à effet Doppler. Techniques utilisées. 
A titre d'exemple, la figure 17 représente ces trois types de base de sondes, mais il est évident qu'il en existe une grande variété de chaque type selon la mesure effectuée.

En ce qui concerne les caractéristiques principales des fils et films chauds, le tableau VII indique à titre d'exemple celles du matériel Dantec (page 418).

Comme le montre ce tableau, la variété est importante et il en résulte des domaines d'application très différents selon le fluide, la vitesse, la température, etc.

Quant aux possibilités offertes par l'anénométrie à fil chaud, elles vont au-delà des mesures de vitesse. On peut en effet également déterminer le taux de turbulence, le tenseur de Reynolds, des corrélations spatiales et spatiotemporelles, ainsi que le spectre d'énergie.

A titre d'exemple des possibilités de l'anémométrie à fil chaud, nous citerons un cas particulier d'application [11]. Il s'agissait d'étudier les écoulements, avec inversion de sens, qui prenent naissance dans une roue mobile de turbomachine, lorsque celle-ci décroche et fonctionne en régime de décollement tournant. Pour une telle étude, conduite au VKI, une sonde spéciale a été réalisée (figure 18). Cette sonde se compose de deux fils parallèles séparés d'une faible distance de $0,6 \mathrm{~mm}$. Le fil $\mathrm{n}^{\circ} 1$ fonctionne avec un rapport de surchauffe de 1,6 tandis que le fil $\mathrm{n}^{\circ}$ 2 opère avec un rapport de 1,1; ce qui augmente la sensibilité aux inversions de sens de l'écoulement. Le fil $\mathrm{n}^{\circ} 2$ se trouvant par exemple dans le sillage du fil $\mathrm{n}^{\circ} 1$, le rapport des vitesses $U 2 / U 1$ est inférieur à l'unité. S'il y a inversion de sens de l'écoulement $U 2 / U 1$ devient plus grand que 1. Moyennant un étalonnage avec vitesse, incidence et pente variables, il est donc possible de remonter aux caractéristiques instantanées de l'écoulement.

Dans le cas de cette étude, la sonde était montée à l'extrados d'une aube à environ mi-corde et mi-envergure et les signaux transmis par contacteur tournant à mercure. La figure 19 montre le schéma de l'ensemble de la chaîne de mesures. Une autre expérience du même genre a d'ailleurs été conduite à l'Ecole centrale lyonnaise [12].

\subsection{Vélocimètre laser}

Cette technique récente de mesure de la vitesse d'un écoulement repose sur l'exploitation de l'effet Doppler connu depuis longtemps [10]. Toutefois une telle application n'a pu se faire que grâce à l'apparition des lasers dont la cohérence temporelle était indispensable pour détecter des variations relatives de fréquence optique très faibles.

D'autre part le laser permet d'obtenir une forte énergie lumineuse dans un très petit volume, ce qui permet :

- d'effectuer des mesures ponctuelles avec une très bonne résolution spatiale du volume de mesure,

- de recueillir la lumière diffusée par des particules microscopiques qui seront supposées suivre parfaitement l'écoulement,

- de ne pas perturber l'écoulement par l'apport d'une sonde matérielle,

- de faciliter les mesures dans les parties mobiles de certaines machines.

Le principe de l'anémométrie laser à effet Doppler est basé sur la mesure du décalage de la fréquence de la lumière émise par une particule en mouvement dans un fluide, éclairée par une onde lumineuse monochromatique.

Une première technique d'application de ce principe est la technique à franges qui consiste à combiner deux rayons incidents qui vont illuminer simultanément la même particule traversant le volume de mesure. Un phénomène d'interférence apparaît et le battement résultant des deux fréquences de la lumière diffusée provenant de chaque faisceau est directement proportionnel à la vitesse de la particule donnée par:

$$
V=\frac{\lambda}{\sin \frac{\theta}{2}} \cdot f_{D}
$$

$V=$ composante de la vitesse mesurée dans le plan des faisceaux perpendiculairement à la bissectrice de l'angle $\theta$ de ces deux faisceaux.

$\lambda=$ longueur d'onde de la lumière laser

$f_{D}=$ fréquence Doppler

Selon le type d'application et les possibilités d'implantation d'un tel système, plusieurs techniques ont été développées [13] comme le montre la figure 20 :

- diffusion avant qui permet d'obtenir le meilleur rapport signal/bruit, mais nécessite de pouvoir traverser une veine,

- rétrodiffusion, généralement la seule possible dans les turbomachines. L'intensité rétrodiffusée est nettement plus faible que celle diffusée vers l'avant et cette technique nécessite l'emploi d'un laser plus puissant et d'une plus grande concentration de particules.

- lorsque deux composantes perpendiculaires de la vitesse sont souhaitées, on utilise un vélocimètre bidimensionnel utilisant, par exemple, les raies vertes et bleues $(5145 \AA$ et $4883 \AA$ ).

Pour déterminer la direction de l'écoulement, on équipe l'optique d'émission de cellules de Bragg qui permettent le défilement des franges d'interférences et donc, de déterminer le sens de l'écoulement.

Sans rentrer dans le détail du traitement de l'information émise par le photodétecteur, nous indiquerons que celui-ci peut être effectué, après amplification et filtrage, par comptage ou corrélateur de photons. Des techniques particulières permettent d'autre part de valider les mesures de fréquence Doppler effectuées, et d'éliminer les signaux émis par les grosses particules qui ne se déplacent pas à la vitesse du fluide.

Les mesures sont généralement mises en mémoire dans une chaîne d'acquisition et de traitement, puis répétées un certain nombre de fois et moyennées.

Dans le cas de mesures à l'intérieur d'une roue mobile de compresseur ou de pompe, il faut adjoindre un dispositif de synchronisation pour piloter la position du point de mesure par rapport aux profils voisins.

En ce qui concerne le volume du domaine de mesure, il est fonction de la focale de l'optique, du diamètre du faisceau laser, de la longueur d'onde de la lumière émise et de l'angle des deux faisceaux.

Un ordre de grandeur est de $200 \mu \mathrm{m}$ pour le diamètre et 7 à $800 \mu \mathrm{m}$ pour la longueur du domaine. 


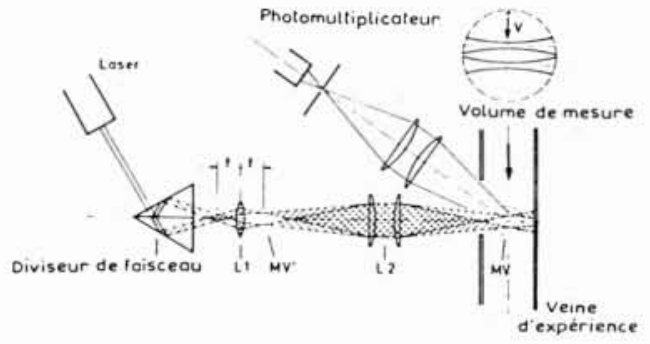

21 . Vélocimètre bi-point.

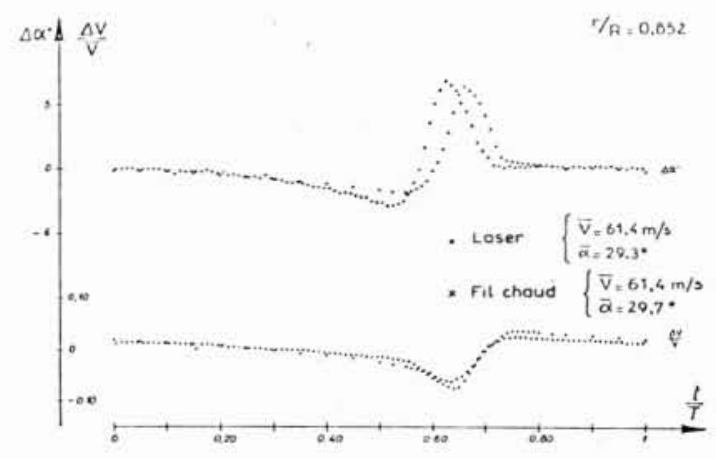

22. Comparaison de mesures de vitesse et de direction de l'écoulement en aval d'une roue mobile de compresseur.

SECTION A.A
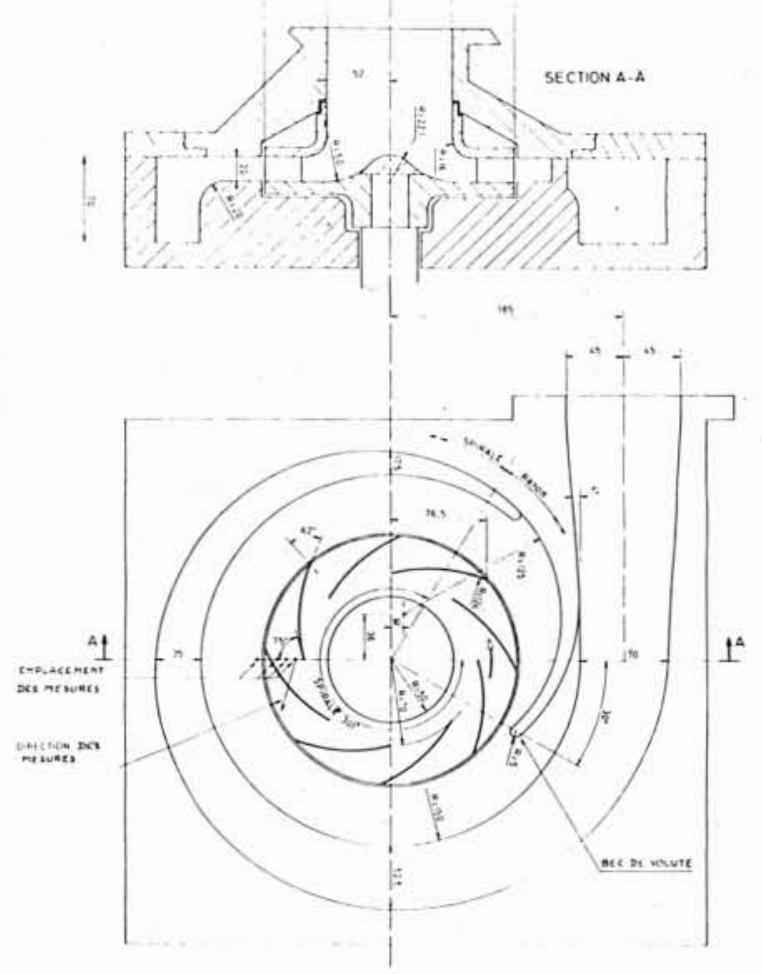

23. Schéma de la pompe à aubes radiales.

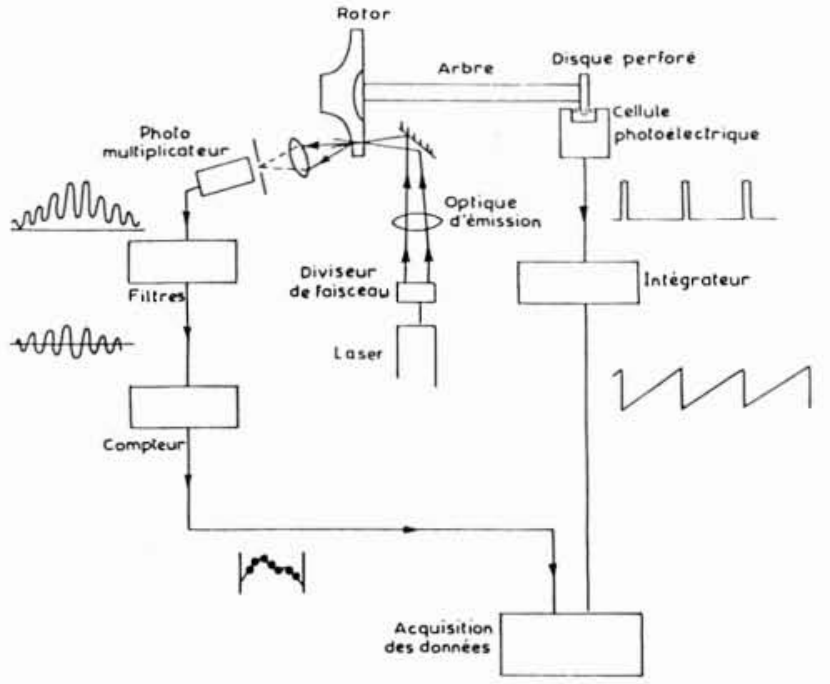

24. Schéma de la chaine de mesure.

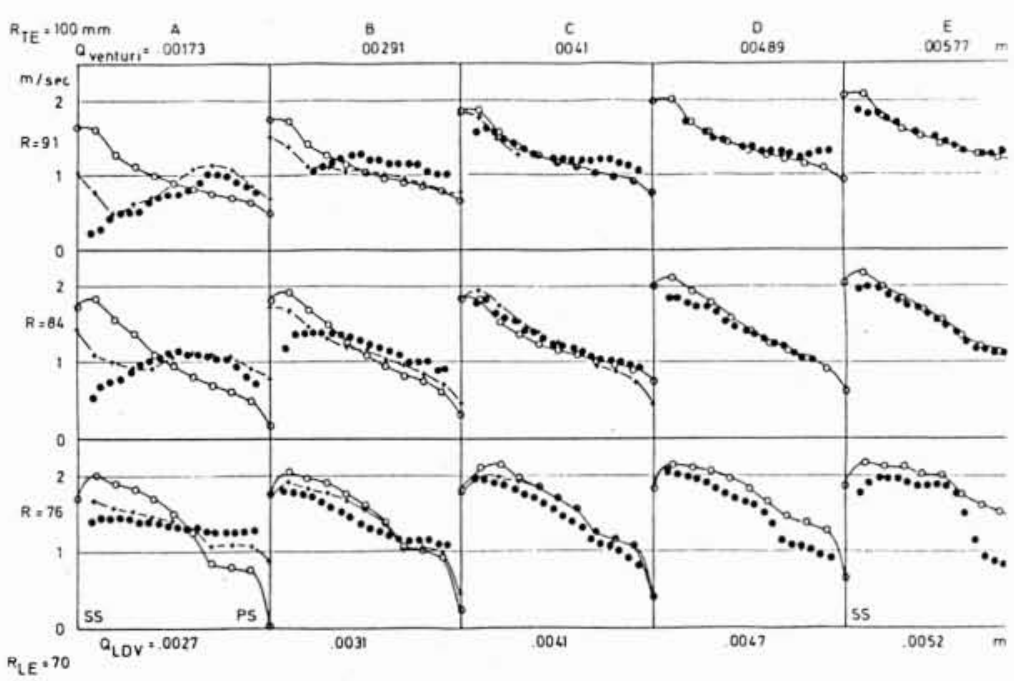

-.. Mesures $\rightarrow$ theorie potentielle $\quad-t-$ Echoppement tourbillonnai

25. Evolution de la vitesse à mi-envergure.

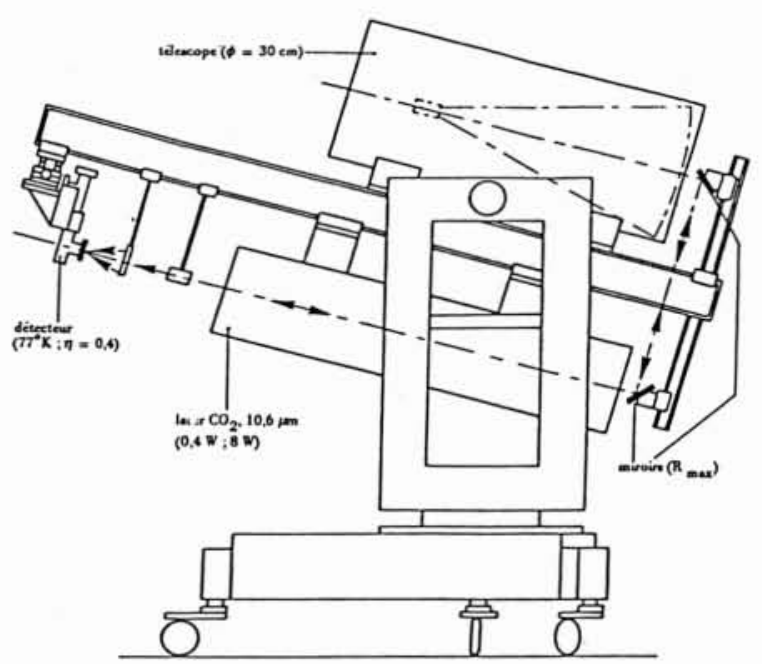

26. Schéma du prototype opérationnel de l'anémomètre à laser. 
La méthode nécessitant la présence de particules, il faut ensemencer, et pour obtenir des particules de diamètre inférieur à $1 \mu \mathrm{m}$, on utilise des aérosols à base d'huile et de solvant pour des températures inférieures à 300 " $\mathrm{C}$ [14]. Lorsqu'il s'agit de faire des mesures dans des écoulements à température plus élevée l'ensemencement est effectué avec des particules solides comme le dioxyde de titane, de silicium ou l'alumine.

Des résultats très intéressants ont été obtenus sauf lorsqu'il s'agit d'effectuer des mesures par rétrodiffusion à proximité d’une paroi (quelques $\mathrm{mm}$ ). Dans ce cas, le rapport signal/bruit devient insuffisant et c'est un inconvénient pour explorer l'écoulement dans certaines machines comme, par exemple, l'écoulement à la sortie d'un rotor de compresseur centrifuge où la veine est souvent peu profonde.

Un autre type de vélocimètre laser permet toutefois de résoudre ce problème. Il s'agit du vélocimètre bipoint [15], dont le principe est indiqué sur la figure $2 l$.

Un module optique permet de focaliser toute l'énergie du laser en deux points très petits $(\sim 10 \mu \mathrm{m})$ espacés de quelques centaines de microns. La mesure consiste alors en une mesure de temps de vol que met une particule pour aller d'un point à l'autre.

Le gain en rapport signal/bruit provient de la concentration accrue de l'énergie lumineuse et de la limitation de la lumière parasite au niveau de la réception.

Les temps d'acquisition sont plus élevés car pour obtenir la direction de la vitesse, il est nécessaire de faire des acquisitions pour plusieurs orientations de la ligne passant par les deux points.

Le vélocimètre bi-point permet une approche frontale de paroi de l'ordre de $0,5 \mathrm{~mm}$ et nécessite l'emploi d'un laser de puissance moyenne $(2,5 \mathrm{~W})$. Pour les mesures sur machines tournantes, il est équipé d'un système de synchronisation à mode multifenêtres.

En conclusion, chaque type de vélocimètre a ses avantages et ses inconvénients, chacun ayant un domaine d'application spécifique.

Un tel appareil de mesure de la vitesse et de la turbulence a un très vaste champ d'utilisation, que ce soit dans les gaz comme dans les liquides, que les vitesses soient faibles ou élevées, et que les températures le soient également.

A titre d'exemple, la figure 22 compare les variations de vitesse et de direction de l'écoulement en aval d'une roue mobile de compresseur mesurées, sur un pas des aubes, par vélocimètre laser à franges et par fil chaud [16]. La comparaison est très satisfaisante sauf au voisinage du sillage,

Un autre exemple concerne une pompe à aubes radiales [17], schématisée sur la figure 23, et dont la figure 24 montre la chaine d'instrumentation. La figure 25 montre l'évolution, à mi-envergure des aubes, de la vitesse tangente à l'aube, entre deux aubes voisines, à différents rayons dans la roue et pour différents débits ou points de fonctionnement. On notera que dans l'ensemble le débit déduit des mesures laser recoupe bien le débit mesuré par venturi. D'autre part, la comparaison théorie-expérience représentée sur la figure donne de bons résultats d'ensemble, sauf pour le point de fonctionnement $A$ qui est proche du décrochage de la machine.
Un autre exemple se rapproche de celui exposé comme application de l'anémométrie à fil chaud [18]. Il s'agit de l'utilisation de l'anémométrie laser à l'étude d'une couche limite décollée dans laquelle existent des phénomènes de recirculation pour la mesure desquels les auteurs préconisent l'emploi du laser plutôt que celui du fil chaud.

Outre ces applications de la vélocimétrie laser à des écoulements gazeux ou liquides, la méthode est intéressante également dans le cas de milieux anisotropes [19]. Toutefois, dans ce type de mesure, les faisceaux lumineux et les trajets optiques sont influencés par la variation de l'indice de réfraction des milieux traversés.

Ainsi, pour répondre au besoin de mesures fines de vitesses dans des écoulements liquides présentant une densité variable en raison de gradients, de salinité ou de température, EDF a développé des vélocimètres laser à sonde plongée dans l'écoulement [20]; les trajets optiques ne sont plus influencés, tandis que les perturbations apportées par la sonde à l'écoulement n'influent pratiquement pas sur la mesure. Aujourd'hui les spécialistes en écoulement d'EDF utilisent couramment ces appareils. Si leur principe est conservé ils bénéficient d'améliorations optroniques et mécaniques, comme par exemple l'utilisation de modulateurs acousto-optiques pour discriminer le signe de la vitesse mesurée.

Enfin, pour compléter ces quelques exemples d'application, nous signalerons un anémomètre à laser infrarouge développé à l'EDF, destiné à des mesures de vitesse et principalement de turbulence atmosphérique dans les basses couches [21]. Pour de telles mesures, le montage utilisé diffère des précédents. On utilise un Michelson dans lequel on remplace l'un des miroirs par un télescope. Une distance de focalisation, largement variable (50 à 500 $\mathrm{m})$ est possible par déplacement du miroir secondaire.

La figure 26 montre le schéma particulier d'un tel dispositif.

\subsection{Corrélation}

Les méthodes directes, précédemment décrites, de mesures de vitesse ne sont pas toujours utilisables.

Au cours de ces dernières années, grâce au développement des dispositifs de traitement numérique du signal, des méthodes de corrélation ont été développées qui fournissent un puissant moyen indirect de mesurer les vitesses [12].

Le principe de la mesure est le suivant : deux détecteurs espacés d'une distance $\mathrm{AB}$ fourniront, en théorie, deux versions du même signal décalées d'un temps $\theta$ qui représente le temps de transit du mobile ou d'une tranche de fluide entre les détecteurs $A$ et $B$.

Connaissant $\mathrm{AB}$ et mesurant $\theta$, on calcule facilement la vitesse moyenne entre $A$ et $B$, parallèlement à $A B$.

Le problème est donc d'estimer le retard $\theta$ entre deux signaux $x(t)$ et $y(t)$ reçus par les détecteurs A et B. C'est l'objet des méthodes de corrélation.

On calcule la fonction d'intercorrélation $C x y(\tau), \tau$ étant le décalage pour lequel les deux signaux se ressemblent le plus. Il y a intérêt à ce que les signaux comportent de nombreux accidents, c'est-à-dire une bande passante relativement large: la superposition en est facilitée. 
Tableau VIII

\begin{tabular}{|c|c|c|c|}
\hline \multicolumn{4}{|c|}{ Domaine d'application de la méthode de corrélation } \\
\hline type d'écoulement & $\begin{array}{l}\text { signaux utiles } \\
\text { capteurs }\end{array}$ & avantages & inconvénients \\
\hline $\begin{array}{l}\text { - liquides monophasi- } \\
\text { ques } \\
\text { - gaz }\end{array}$ & - radioéléments & $\begin{array}{l}\text { non-intrusif } \\
\text { capteur sans contact }\end{array}$ & $\begin{array}{l}\text { éventuelle sensibilité au profil } \\
\text { des vitesses }\end{array}$ \\
\hline $\begin{array}{l}\text { - liquide monophasi- } \\
\text { que }\end{array}$ & - ultrasons & $\begin{array}{l}\text { non-intrusif } \\
\text { bande passante élevée } \\
\text { temps de mesure relativement } \\
\text { court }\end{array}$ & - sensibilité au profil des vitesses \\
\hline - liquides, gaz & - température & bande passante assez large & - intrusif \\
\hline - vitesse de vagues & $\begin{array}{l}\text { signal optique (cellule } \\
\text { photo- électrique) }\end{array}$ & $\begin{array}{l}\text { sans contact } \\
\text { bonnes caractéristiques des si- } \\
\text { gnaux }\end{array}$ & \\
\hline $\begin{array}{l}\text { - vitesse de feuille de } \\
\text { papier }\end{array}$ & $\begin{array}{l}\text { vélocimètre laser sur surface } \\
\text { diffusante }\end{array}$ & $\begin{array}{l}\text { très large bande passante } \\
\text {. temps de mesure court } \\
\text {. mesure sans contact }\end{array}$ & \\
\hline - Eléments gazeux & - anémomètres à fil chaud & $\begin{array}{l}\text { bande passante très grande } \\
(\sim 10 \mathrm{kHz}) \\
\text {. temps de réponse très bref }\end{array}$ & $\begin{array}{l}\text { nécessité d'avoir une très } \\
\text { grande précision de position- } \\
\text { nement des capteurs }\end{array}$ \\
\hline $\begin{array}{l}\text { - liquides } \\
\text { - métaux liquides }\end{array}$ & - sondes électromagnétiques & - application au sodium liquide & $\begin{array}{l}\text { sensibilité au profil des vitesses } \\
\text { temps de réponse assez long }\end{array}$ \\
\hline $\begin{array}{l}\text { - liquide chariant des } \\
\text { particules solides }\end{array}$ & $\begin{array}{l}\text { capteurs optiques ou capaci- } \\
\text { tifs }\end{array}$ & . non-intrusif & $\begin{array}{l}\text { caractéristiques du signal peu } \\
\text { élevées } \\
\text { - sensibilité au profil des vitesses }\end{array}$ \\
\hline
\end{tabular}

Dans les cas particuliers de signaux contenant des composantes périodiques, il y a intérêt à remplacer la fonction d'intercorrélation par la fonction de cohérence entre $x(t)$ et $y(t)$.

Ces méthodes sont très intéressantes et ont donné des résultats très satisfaisants en laboratoire. Il faut toutefois préciser qu'elles ne s'appliquent qu'à des écoulements stables fournissant des signaux stationnaires.

Quant au champ d'application, il est très vaste, comme le montre le tableau VIII ci-après qui indique également la grande variété de signaux utilisés.

\subsection{Analyse d'image}

La méthode d'analyse d'image permet de mesurer la vitesse dans des écoulements, à condition qu'ils contiennent des particules visualisables [23].

Le principe consiste à explorer le champ intéressé par une caméra Vidicon, analyser en ligne et mémoriser les contours significatifs sur une mémoire de masse et à

Tableau IX

\begin{tabular}{|l|l|}
\hline \multicolumn{2}{|c|}{ Performances de l'analyseur d'image } \\
\hline $\begin{array}{l}\text { Définition } \\
\text { Temps d'analyse et mémorisation } \\
\text { d'une image }\end{array}$ & $800 \mathrm{pts} \times 1000$ lignes \\
$\begin{array}{l}\text { Nombre d'images enregistrables à } \\
\text { la cadence max }\end{array}$ & $\simeq 50$ \\
\hline
\end{tabular}

effectuer le traitement permettant de remonter aux déplacements d'une image à l'autre par calculateur.

Les performances sont indiquées dans le tableau IX.

\subsection{Sodar}

Le "sodar " est un instrument de télémesure qui permet de sonder de façon continue les basses couches de l'atmosphère [24]. Il fournit des informations numérisées sur le vent (répartition des directions et des vitesses suivant la verticale) et un certain nombre de paramètres que l'on peut relier à la structure thermique et à la turbulence.

Son fonctionnement est basé sur la rétrodiffusion d'un signal sonore par les cellules de turbulence thermique éventuellement présentes dans l'atmosphère et ayant des dimensions de l'ordre de la demi-longueur de l'onde émise, soit une dizaine de centimètres pour une fréquence d'émission de $1600 \mathrm{~Hz}$.

L'appareil se compose de 3 antennes orientées différemment (figure 27).

Elles fonctionnent à la fois comme émetteur et comme récepteur et sont interrogées de façon cyclique.

Le signal rétrodiffusé est analysé :

- en amplitude, d'où la distribution verticale des inhomogénéités en température,

- en fréquence, d'où les composantes du vecteur vent.

Un micro-ordinateur pilote l'unité d'émission-réception et l'unité de traitement du signal.

Un tel appareil, construit par Bertin, fonctionne depuis 1979 à St Alban. 


\section{Mesures de débit}

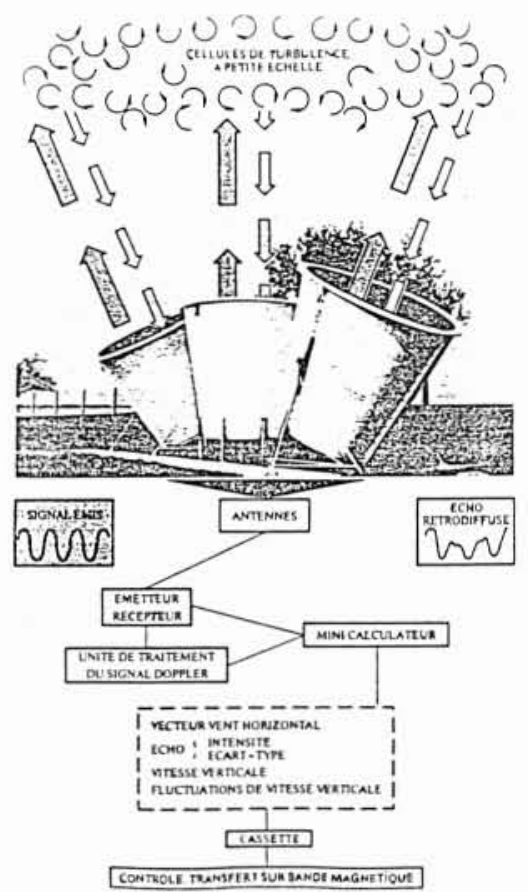

Les mesures de débit dérivent souvent de mesures de vitesses et le développement d'appareillages nouveaux pour la détermination de la vitesse se répercute sur les mesures de débit, principalement dans le domaine des mesures transitoires.

Bien que certaines techniques sont connues et utilisées depuis longtemps, des améliorations leur ont été apportées qu'il est bon de signaler. D'autres sont entièrement récentes. Ces méthodes sont classées dans le tableau X.

Tableau $\mathbf{X}$

\begin{tabular}{|ll|}
\hline \multicolumn{1}{|c|}{ Méthodes de mesure du débit } \\
\hline $4-1$ & Moulinet \\
$4-2$ & Débitmètre électromagnétique \\
$4-3$ & Débitmètre à ultrasons \\
$4-4$ & Débitmètre à vortex \\
$4-5$ & Débitmètre massique \\
$4-6$ & Traceurs radioactifs - Corrélation \\
$4-7$ & Traceurs radioactifs - Temps de transit \\
\hline
\end{tabular}

\subsection{Moulinet}

Cet appareil utilisé depuis longtemps pour la mesure des débits par mesure des vitesses a subi, au cours de ces dernières années, des modifications sensibles. Cette modernisation, [25] effectuée à EDF-DTG, a consisté essentiellement à remplacer les collecteurs des moulinets par des interrupteurs magnétiques de type ILS et à réaliser une centrale de mesure pilotée par minicalculateur, traitant les signaux correspondants, ce qui permet d'analyser de façon quasi immédiate les résultats.

La figure 28 montre un ensemble pour la mesure du débit en conduite forcée et le schéma synoptique correspondant d'acquisition et de traitement des données.

Les caractéristiques de l'appareil sont indiquées dans le tableau XIa.

Tableau XIa

\begin{tabular}{|c|c|}
\hline - Nombre de voies & 100 \\
\hline Signal "contact" & $\begin{array}{l}\text { tension: réglable de } 0 \text { à } 7 \mathrm{~V} \\
\text { efficaces } \\
\text { fréquence : } 225 \mathrm{~Hz} \pm 1 \mathrm{~Hz} \\
\text { puissance du générateur: } 7 \mathrm{VA}\end{array}$ \\
\hline - Horloge & $\begin{array}{l}\text { Résolution } 0,01 \mathrm{~s} \\
\text { Capacité d'intégration } 999,99 \mathrm{~s}\end{array}$ \\
\hline - Vitesse d'exploration & $\begin{array}{l}\text { environ } 20 \text { voies/seconde (avec } \\
\text { HP 9826) }\end{array}$ \\
\hline - Présentation & $\begin{array}{l}10 \text { blocs de comptage de } 10 \text { voies } \\
1 \text { bloc de commande } \\
\text { dimensions d'un bloc: } \\
51 \times 37 \times 30 \mathrm{~cm} \\
\text { poids : bloc de commande } 19 \mathrm{~kg} \\
\text { bloc de comptage } 18 \mathrm{~kg}\end{array}$ \\
\hline - Alimentation & $\begin{array}{l}\text { bloc de } 10 \text { voies } \\
220 \mathrm{~V} \pm 10 \% 45 \mathrm{VA}\end{array}$ \\
\hline & $\begin{array}{l}\text { bloc de commande } \\
220 \mathrm{~V} \pm 10 \% 70 \mathrm{VA}\end{array}$ \\
\hline
\end{tabular}




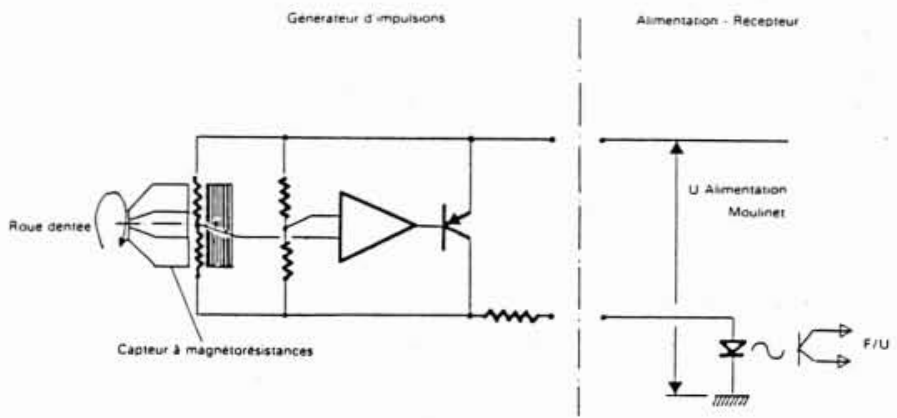

29. Schéma de principe du moulinet pour mesures en transitoire.
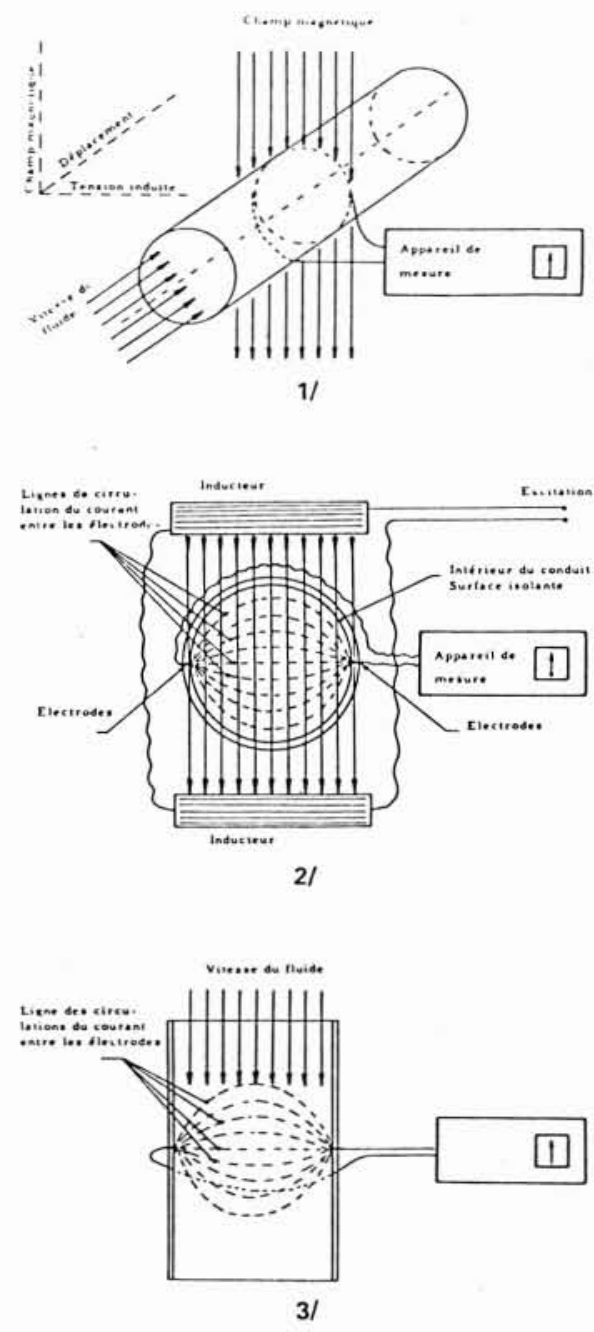

31. Schéma de principe du débitmètre électromagnétique.

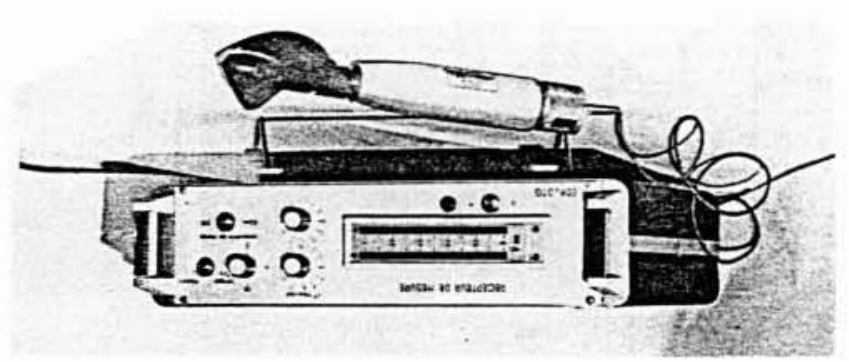

30. Vue d'un ensemble de mesure.

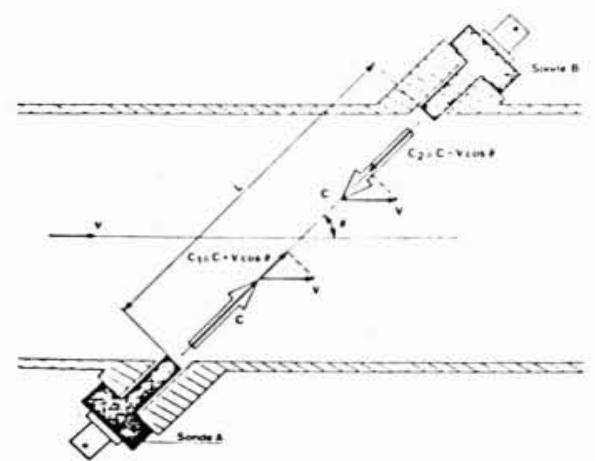

32. Principe du débitmètre à ultrasons type "mesure par différence de temps de parcours".

Par ailleurs, pour pouvoir réaliser des mesures de débit en régime transitoire dans un cas bien particulier, des moulinets ont subi quelques adaptations sous la forme de l'utilisation d'hélices légères en plastique associées à un capteur de vitesse angulaire. La figure 29 en montre le schéma de principe et la figure 30 présente un ensemble de mesure.

Les caractéristiques principales de ces moulinets à magnétorésistances sont indiquées dans le tableau XIb.

\section{Tableau XIb}

\begin{tabular}{|lc|}
\hline \multicolumn{2}{|c|}{ Caractéristiques des moulinets à magnétorésistance } \\
\hline Accélération d'écoulement mesurable & $0,5 \mathrm{~ms}^{-2}$ \\
(avec précision de $5 \%$ ) & \\
Vitesse maximum avec une hélice au pas de & $5 \mathrm{~ms}^{-1}$ \\
$0,25 \mathrm{~m}$ & $+15 \mathrm{~V}$ \\
Tension d'alimentation du capteur & 12 \\
\hline Nombre de capteurs traités simultanément & \\
\hline
\end{tabular}




\subsection{Débitmètre électromagnétique}

La mesure des débits par méthode électromagnétique est également ancienne, mais c'est grâce aux progrès des composants électroniques, au cours de ces dernières années, que des matériels sûrs et fiables ont pu être réalisés.

Nous en rappelerons brièvement le principe :

- tout conducteur en mouvement dans un champ magnétique est le siège d'une force électromagnétique induite :

$$
E=K d \phi / d t
$$

force représentative de la vitesse du fluide.

La figure 31 schématise le principe de fonctionnement d'un tel débitmètre [26] qui toutefois, ne s'applique qu'aux liquides dont la conductibilité électrique est supérieure à $1 \mu \Omega \mathrm{cm}^{-1}$. Ce qui exclut les huiles, essences et eaux très pures.

Ce type d'appareil existe depuis des diamètres de l'ordre de $10 \mathrm{~mm}$ jusqu'à environ 3 mètres. Toutefois, les appareils de grandes tailles sont coûteux, moins précis et l'étalonnage indispensable devient délicat.

Le niveau de vitesse recommandé pour le fluide circulant dans le débitmètre est de 1 à $10 \mathrm{~m} / \mathrm{s}$.

Cette technique de mesure est actuellement en cours de développement en vue de son application aux eaux naturelles et aux eaux fortement chargées (elle est très répandue pour les mesures de débit de métaux liquides). De même les niveaux de pression et de température de fonctionnement s'accroissent. Enfin, il faut signaler l'intérêt, encore peu exploité, de cette méthode pour la mesure des vitesses et des débits fluctuants.

\subsection{Débitmètres à ultrasons}

Le débitmètre à ultrasons est destiné à la mesure des débits de liquides en conduite fermée où à la mesure des vitesses en écoulement libre [27].

Le principe de fonctionnement est le suivant : deux sondes ultrasonores (alternativement émettrices et réceptrices), placées de part et d'autre de la canalisation, sur une ligne oblique par rapport à la direction de l'écoulement émettent en synchronisme des trains d'ondes acoustiques (figure 32 ).

L'entraînement des ondes ultrasonores par le mouvement du liquide a pour effet de modifier les temps de propagation entre sondes. Le déphasage entre les signaux reçus représente la vitesse moyenne du liquide dans la veine. Les signaux sont traités pour délivrer des informations de débit volumique instantané et de volume totalisé. La composition vectorielle de la célérité du son $C$ dans le liquide et de la vitesse d'écoulement $V$ de celui-ci, met en évidence une célérité apparente du son:

$$
\begin{aligned}
& C_{1}=C+V \cos \theta(\text { de } A \text { vers } \mathrm{B}) \\
& C_{2}=C-V \cos \theta(\text { de } \mathrm{B} \text { vers } \mathrm{A})
\end{aligned}
$$

Si $T_{1}$ et $T_{2}$ sont les temps de propagation correspondants, pour la distance $L$ séparant les deux capteurs, on en déduit :

$$
V=\frac{L}{2 \cos \theta} \frac{T_{2}-T_{1}}{T_{1} \cdot T_{2}}
$$

La mesure est indépendante des variations de la célérité du son dans le milieu.

Il s'agit là d'une mesure par différence de temps de parcours. On peut également utiliser un procédé par différence de fréquence ou "sing around " [28].

En ce qui concerne les avantages de ces appareils, ils sont nombreux :

- les capteurs sont statiques,

- la veine n'est pas perturbée,

- ils n'introduisent pas de pertes de charge,

- l'écoulement peut circuler dans les deux sens,

- ils sont compatibles avec tous les liquides,

- la température du fluide n'a pas d'influence,

- la bande passante est élevée,

\begin{tabular}{|c|c|}
\hline \multicolumn{2}{|c|}{ Performances des débitmètres à ultrasons } \\
\hline Etendue de mesure & Quelques $\frac{\mathrm{cm}^{3} \mathrm{~s}^{-1} \text { à quelques }}{\mathrm{m}^{3} \mathrm{~s}^{-1}}$ \\
\hline $\begin{array}{l}\text { Précision } \\
\text { Résolution }\end{array}$ & $\begin{array}{l}0,2 \% \text { de l'étendue de mesure } \\
\text { Théoriquement infinie }\end{array}$ \\
\hline $\begin{array}{l}\text { Bande passante } \\
\text { (type mesure des différen- } \\
\text { ces de temps de parcours) }\end{array}$ & $\begin{array}{l}\text { jusqu'à } 20000 \text { mesures par } \\
\text { seconde pour les plus récents } \\
\text { [29] }\end{array}$ \\
\hline Domaine de température & $-200^{\circ} \mathrm{C}$ a $+300^{\circ} \mathrm{C}$ \\
\hline
\end{tabular}

- la pression a très peu d'influence,

- la gamme des canalisations est très large.

Tableau XII

Afin de pouvoir effectuer des mesures de débit sur des installations existantes, sans avoir à y apporter des modifications quasi impossibles, des débitmètres à ultrasons " accrochables" sont en cours de développement.

Au point de vue applications, plusieurs, de type industriel, ont été effectuées par EDF. Nous citerons par exemple :

- à l'usine de haute chute de Sassenage, à l'aide d'un débitmètre du type "mesure par différence de fréquence ", représenté schématiquement sur la figure 33 (page suiv.); au cours de deux ans et demi d'exploitation, les écarts de débit (par rapport au débit thermodynamique) ont été très faibles,

- sur l'aménagement Franco-Suisse d'Emosson qui comprend 6 débitmètres du type "mesure des différences de temps de parcours ", la précision des mesures effectuées est conforme aux garanties du constructeur et les appareils très fiables.

Avant de terminer sur ce chapitre, il faut toutefois signaler quelques problèmes liés au principe de la mesure par ultrasons.

La mesure est proportionnelle à la vitesse moyenne de l'écoulement dans le plan diamétral défini par les deux transducteurs et non à la vitesse débitante. Pour accéder à celle-ci, les constructeurs proposent un coefficient correctif qui prend généralement en compte le profil du champ de vitesse théorique de Nikuradse. Or, les écarts entre profil théorique et profil réel peuvent être importants malgré les précautions d'implantation préconisées qui prévoient un alignement droit de 10 diamètres à l'amont et 5 diamètres à l'aval. L'utilisation de plusieurs plan de mesure ne permet pas toujours de résoudre ce problème. 


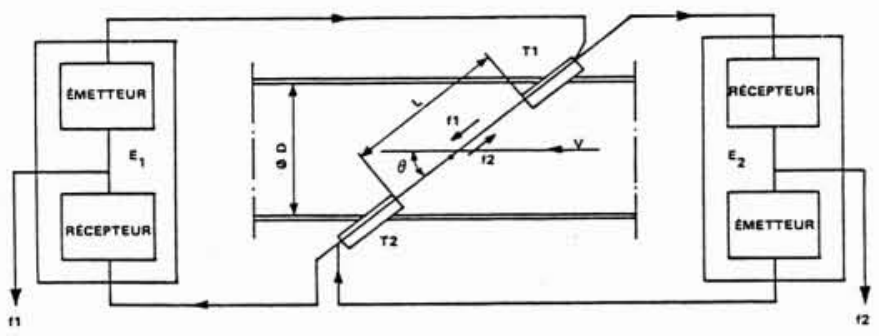

33. Débitmètre du type "mesure par différence de fréquence ".

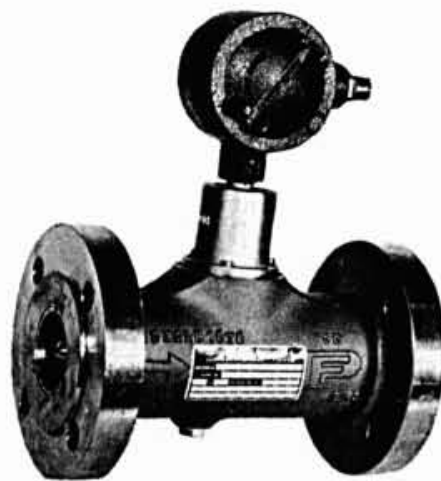

34. Vue d'un débitmètre piézo-précessif.

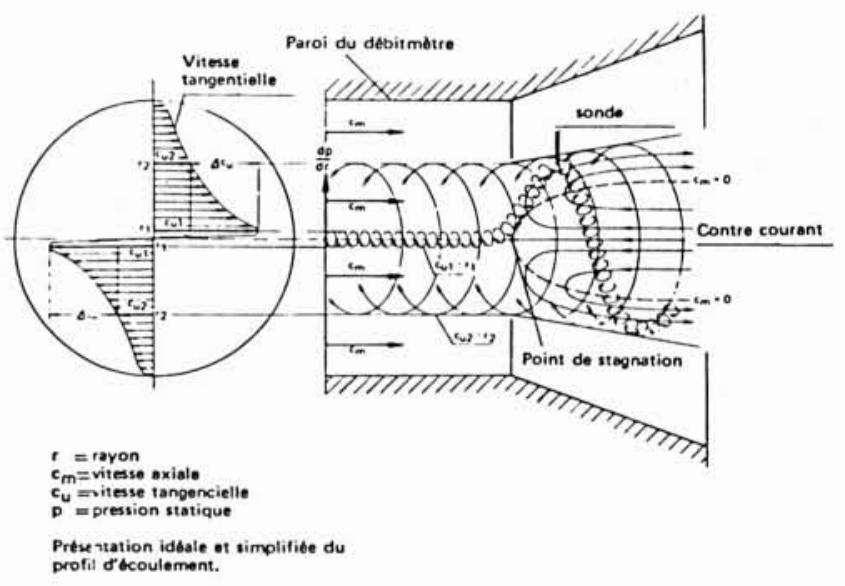

35. Principe du débitmètre piézo-précessif.

\subsection{Débitmètre à vortex}

Les débitmètres "à vortex " sont adaptés à la mesure des débits de gaz et de liquide.

Ils utilisent les tourbillons qui peuvent se former, de façon périodique, dans certains écoulements.

Ainsi, un barreau perpendiculaire à l'écoulement engendre dans celui-ci une rue de tourbillons alternés (tourbillons de Bénard-Karman) dont la fréquence de détachement est, dans un grand domaine de nombre de Reynolds, proportionnelle à la vitesse du fluide donc à son débit volumique.

De même, dans le débitmètre piézoprécessif [30] dont la figure 34 montre une vue. Le profil de l'aubage d'entrée du débitmètre engendre une composante tangentielle de la vitesse d'où un gradient radial de pression qui se traduit par une instabilité du débit et une rotation secondaire des "vortex" et par des variations relatives de vitesse du fluide (figure 35). Il y a donc précession du "vortex ".

Dans les deux cas, les variations de vitesse sont détectées par une sonde qui crée un signal électrique dont la fréquence est directement proportionnelle au débit volumique.

Selon le type d'application, on utilise :

- soit une sonde sensible aux variations de température (thermistance),
- soit une sonde sensible aux variations de pression, (sonde piézo-électrique).

Quant aux avantages de ces types d'appareils, ils sont nombreux :

- absence de pièces en mouvement,

- utilisables avec gaz humides, sales, poussiéreux ou haute pression,

- montage facile,

- sorties fréquence et analogique permettant l'emploi de systèmes digitaux et de calculateurs.

L'appareil permet des totalisations aussi bien que des indications de débit instantané.

Quant aux principales caractéristiques techniques, elles sont indiquées sur le tableau $X I I I$ :

Tableau XIII

\begin{tabular}{|l|l|}
\hline \multicolumn{2}{|c|}{ Caractéristiques des débitmètres à vortex } \\
\hline $\begin{array}{l}\text { Précision } \\
\text { Fidélité }\end{array}$ & $\pm 1 \%$ de $20 \%$ à $100 \%$ du débit \\
Montage & $\pm 0,25 \%$ du débit maximum instantané \\
& 5 diamètres de longueur droite amont \\
Température diamètres de longueur droite aval \\
$\begin{array}{l}\text { maximale du } \\
\text { fluide }\end{array}$ & -40 à $+110^{\circ} \mathrm{C}$, avec sonde piézo- \\
électrique
\end{tabular}




\subsection{Débitmètre massique}

Ces débitmètres, qui utilisent " les forces de Coriolis", sont de développement récent.

La masse est mesurée directement par un capteur sans obstruction avec une précision de $\pm 0,4 \%$ et un temps de réponse de 0,1 à 1,1 sec. La mesure est insensible aux variations de :

- température

- pression

- viscosité

- densité

- profil d'écoulement.

\subsection{Traceurs radioactifs - Corrélation}

Une mesure de débit de fluide primaire des réacteurs PWR a été expérimentée à EDF [22].

Cette mesure utilise le fait que les noyaux d'oxygène 16 de l'eau du fluide caloporteur (fluide primaire) sont transformés, par activation neutronique, en noyaux d'azote 16, lors de la traversée du cœur du réacteur. Le fluide primaire émet ainsi de la radioactivité gamma qui est détectée par des chambres à ionisation ou des scintillateurs. Le signal utile est la fluctuation temporelle de la concentration en azote 16 des tranches de fluide successives qui passent devant les détecteurs. Après conditionnement, les signaux de sortie de ceux-ci sont correlés. La détermination du maximum de la fonction obtenue donne une mesure du retard entre les deux signaux, et permet donc de remonter au débit moyen.

Cette méthode présente l'avantage d'être non intrusive, puisque les détecteurs sont extérieurs, et de fournir une mesure absolue du débit, sans qu'il soit nécessaire d'étalonner. Elle n'est pas, à ce jour, utilisée sur les réacteurs français.

\subsection{Traceurs radioactifs - Temps de transit}

Cette seconde mesure de débit à l'aide de la détermination du temps de transit de particules radioactives entre deux sections droites éloignées d'une distance connue, est applicable aux conduites fermées [31].

Certaines conditions doivent évidemment être respectées pour l'application d'une telle méthode; elles concernent :

- les positions de l'injection et des détecteurs,

- la configuration de la conduite,

- le nombre de Reynolds qui doit être $\geqslant 5000$,

- le type d'injection,

- l'influence de la vitesse d'écoulement,

- les conditions d'un bon mélange,

- etc.

Le traceur radioactif ( $137 \mathrm{mBa}$ ) choisi émet un rayonnement $\gamma$ détecté par des scintillateurs à iodure de sodium et à la sortie de la chaine de détection, on recueille des impulsions qui sont sélectionnées et comptées et permettent de déterminer le temps de transit, d'où le débit.

Une extension de la méthode aux conduites de grand diamètre a fait l'objet d'études complémentaires. La détection se fait par des détecteurs placés dans des doigts de gants diamétraux [32].

\section{Mesures de température}

Parallèlement aux mesures de pression, il est généralement indispensable d'effectuer des mesures de température, aussi bien au niveau du fluide que de la paroi de la conduite susceptible de transporter ce fluide.

A cet effet, il existe depuis de nombreuses années des matériels et techniques très fiables qui donnent satisfaction. Néanmoins, avec l'apparition de problèmes nouveaux, des adaptations de matériels existants ont du être faites, tandis que des moyens nouveaux ont été développés comme l'indique le tableau XIV.

Tableau XIV

\begin{tabular}{|cl|}
\hline Méthodes de mesure des tempèratures \\
\hline $5-1$ & Sonde résistive \\
$5-2$ & Capteurs pelliculaires \\
$5-3$ & Infrarouge \\
$5-4$ & Fibres optiques \\
$5-5$ & D.R.A.S.C. \\
\hline
\end{tabular}

\subsection{Sonde résistive}

Utilisant une sonde résistive classique (résistance de platine) EDF-DTG a développé un thermomètre digital autonome spécialement adapté à la mesure des températures de l'eau en rivières, canaux ou conduites [33].

Cet appareil délivre un signal de sortie sous forme d'impulsions électriques se prêtant aisément :

- soit à une collecte des données sur un support pouvant permettre un traitement automatique,

- soit à une transmission à distance avec affichage.

Le principe repose sur l'utilisation d'une sonde à résistance de platine de $100 \Omega$ qui est insérée dans un pont de Wheatstone que l'on cherche à équilibrer en commutant en parallèle sur la branche opposée des résistances successives.

Sur ordre, ces résistances, qui sont entre elles dans un rapport binaire, sont commutées par un compteur binaire déclanché par un générateur d'impulsions.

En conséquence, la tension de déséquilibre du pont varie linéairement avec le nombre d'impulsions comptées. Lorsque l'équilibre est atteint, le générateur d'impulsions est stoppé. L'appareil a donc émis un nombre d'impulsions proportionnel à la variation de résistance de la sonde, donc à la température.

L'appareil existe en deux versions :

a) un thermomètre pour enregistreur magnétique digital séquentiel à cassette comme le montre la figure 36 (page suiv.); le but étant la surveillance de l'évolution de la température en un point donné,

b) un thermomètre digital pour "saumon " dont la figure 37 (page suiv.) montre un exemple, le but étant de déterminer la carte des températures d'une section d'un cours d'eau ou d'un canal.

Une variante dite " 4 fils " de ce type de thermomètre a été développée afin de permettre l'interchangeabilité des sondes résistives. 


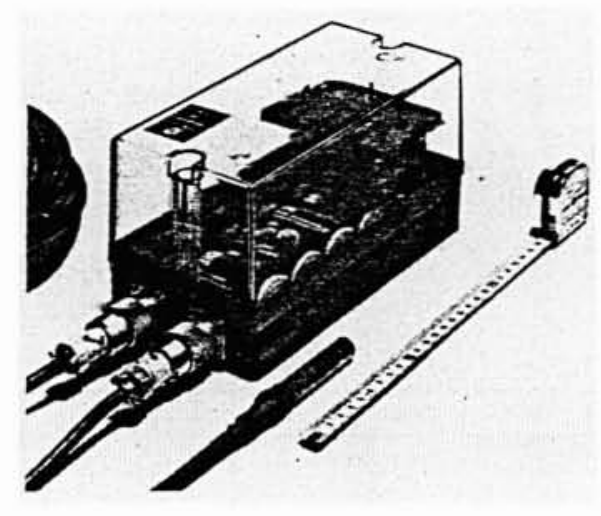

36. Thermomètre digital à sonde résistive.

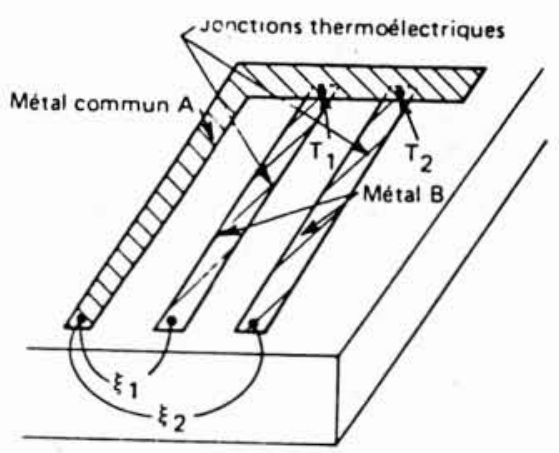

38. Schéma de principe des capteurs pelliculaires de température.

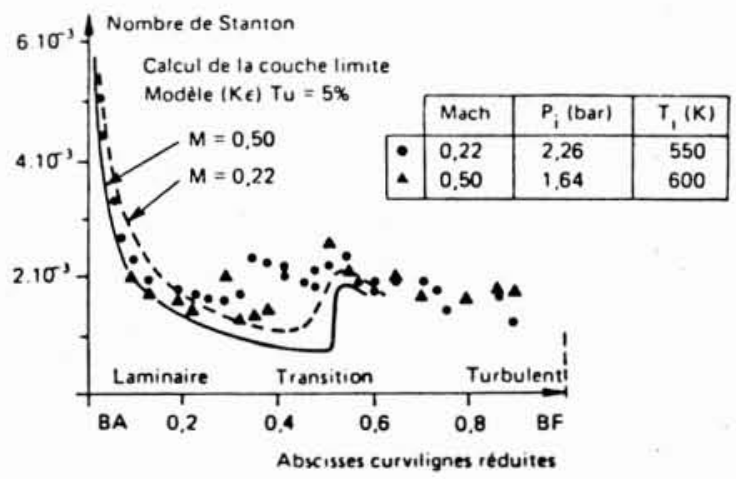

40. Comparaison entre l'évolution du coefficient d'échange théorique, entre le gaz et le profil le long de la corde, et le coefficient déduit des mesures de flux.

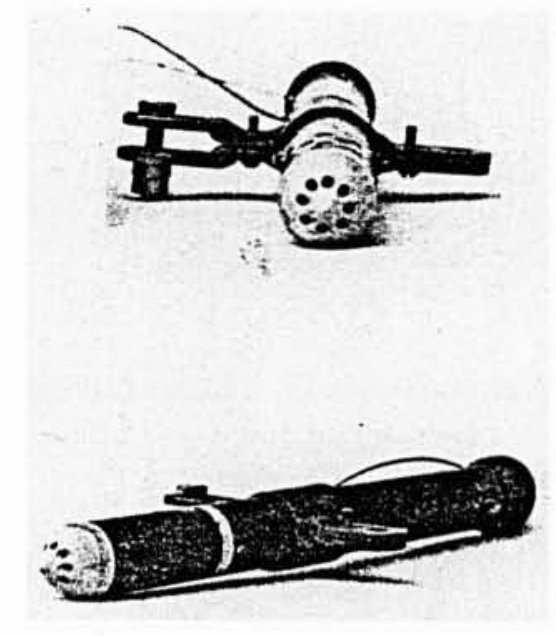

37. Sonde "Saumon ".

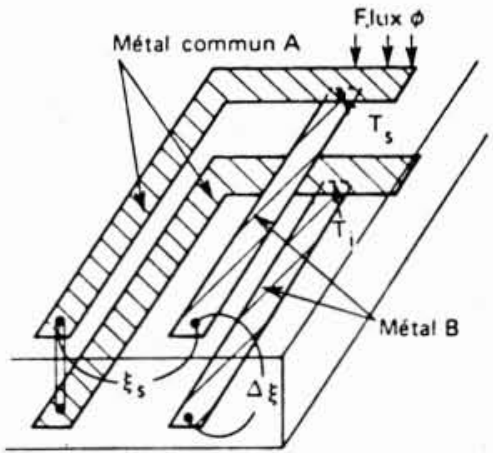

39. Schéma de principe des fluxmètres pelliculaires.

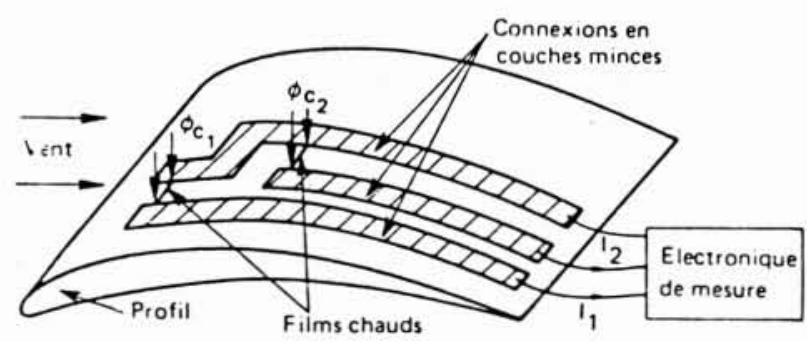

41. Détecteur d'analyse de la couche limite. 
Les caractéristiques de cet appareil sont rassemblées dans le tableau $X V$.

Tableau XV

\begin{tabular}{|ll|}
\hline \multicolumn{2}{|c|}{ Caractéristiques des sondes résistives EDF/DTG } \\
\hline Gamme de mesure & $0-40^{\circ} \mathrm{C}$ \\
Sensibilité & $0,1^{\circ} \mathrm{C}$ \\
Précision & $0,3^{\circ}$ sur l'étendue de \\
& mesure \\
Température de fonctionne- & $-30^{\circ}$ à $+50^{\circ} \mathrm{C}$ \\
ment & Niveau logique code ou \\
Signal de sortie & impulsion à $2,5 \mathrm{Khz}$ \\
& 0 à $250 \mathrm{~ms}$ \\
Temps de réponse & $50 \mathrm{~m}$ \\
Câble de liaison &
\end{tabular}

\subsection{Capteurs pelliculaires}

Les capteurs pelliculaires de température ont été développés à l'ONERA en raison du besoin d'apprécier finement les échanges thermiques au niveau des aubages des turbomachines [4], [34].

Leur développement dérive de celui des capteurs pelliculaires de pression dont nous avons parlé précédemment et ils sont basés sur l'effet thermoélectrique existant à la jonction de deux métaux partiellement superposés et déposés en couche mince sur un support diélectrique, comme le montre la figure 38 .

En plaçant un isolant entre deux thermomètres pelliculaires on peut d'ailleurs, comme le montre la figure 39 , réaliser un fluxmètre à gradient adapté à l'évaluation des échanges convectifs en régime permanent.

En ce qui concerne la réalisation de ces capteurs, dont rappelons-le, l'implantation ne nécessite pas d'usinage, elle est très semblable à celle des capteurs pelliculaires de pression.

Nous indiquerons seulement que c'est le couple cuivre-nickel qui est réalisé lorsque l'on emploie l'évaporation sous vide, tandis qu'avec la pulvérisation cathodique c'est le couple chromel-constantan.

Les caractéristiques de ces thermocouples en couche mince sont rassemblées dans le tableau XVI.

Comme le montre ce tableau, la sensibilité des thermocouples pelliculaires est inférieure à celle des mêmes thermocouples en fils massifs. D'autre part, elle varie d'une fabrication à l'autre.

La particularité des thermocouples en couche mince se traduit par deux conséquences :

- la nécessité de les étalonner,

- la nécessité de soigner particulièrement les points de liaison entre couche mince et fil massif.

Au point de vue application de ces capteurs, nous indiquerons les essais en soufflerie d'un profil symétrique NACA de la série 65 , équipé de 28 fluxmètres pelliculaires disposés le long d'une corde.

Le but de ces essais était d'apprécier l'évolution du coefficient d'échange gaz-profil. La comparaison des résultats avec le calcul théorique est indiqué sur la figure 40.

On notera un bon accord dans la zone laminaire en ce qui concerne l'évolution du nombre de Stanton caractérisant l'échange.

Un troisième type de capteurs pelliculaires thermiques a été développé; il s'agit de capteurs analysant la couche limite d'un écoulement aérodynamique (figure 41).

Ils sont constitués d'un film métallique résistant, déposé sur une feuille de Kapton dont la résistance varie avec la température à laquelle il est soumis.

Le film est chauffé par effet Joule et stabilisé à une température déterminée.

En présence d'écoulement, le film se refroidit par convection et l'échange convectif diminue lorsque l'épaisseur de la couche limite augmente. La valeur de la puissance qui doit être appliquée au film pour le maintenir à température constante, renseigne donc sur sur l'épaisseur de la couche limite.

On peut également évaluer le taux de turbulence de cette couche limite.

\subsection{Infrarouge}

Les caméras IR se divisent en deux familles principales : - à détecteur quantique et balayage optomécanique (les plus répandues) : la résolution est de l'ordre d'une centaine de points par ligne et d'une centaine de lignes par trame. Des mesures de température précises sont possibles;

- à détecteur pyro-électrique et balayage électronique (les moins répandues) : leurs performances inférieures les réservent au contrôle qualitatif des matériels.

La numérisation du signal vidéo et le traitement d'images ont beaucoup évolué. Des logiciels de calibra-

Tableau XV

\begin{tabular}{|c|c|c|c|c|c|}
\hline \multicolumn{6}{|c|}{$\begin{array}{l}\text { Caractéristiques des capteurs pelliculaires de température } \\
\text { Comparaison avec les thermocouples classiques }\end{array}$} \\
\hline \multirow{2}{*}{\multicolumn{2}{|c|}{ Caractéristiques }} & \multicolumn{2}{|c|}{ Cuivre-Nickel } & \multicolumn{2}{|c|}{ Chromel-Constantan } \\
\hline & & Fils & Pelliculaires & Fils & Pelliculaires \\
\hline \multirow{2}{*}{ Coefficients } & $\mathrm{a}, \mu \mathrm{VK}^{-1}$ & 21.89 & 15 & 58.7 & 36.9 \\
\hline & b. $\mu \mathrm{VK}^{-1}$ & $1.54 .10^{-1}$ & $3.18 .10^{-2}$ & $8.62 .10^{-2}$ & $10.2 .10-2$ \\
\hline \multicolumn{2}{|c|}{$\begin{array}{c}\text { Pente de la caractéristique } \\
\text { linéarisée entre } 0 \text { et } 100 \\
\mu \mathrm{VK}^{-1}\end{array}$} & 23.2 & 17.6 & 62.4 & 45.3 \\
\hline \multicolumn{2}{|c|}{ Dispersion de a, \% } & - & \pm 10 & \pm 0.5 & \pm 15 \\
\hline
\end{tabular}


tion, conversion en température à partir d'une équation simplifiée de transfert radiatif, analyse d'image par filtrage, seuillage, codage en fausses couleurs, soustraction ou moyennage d'images, etc. sont commercialisés par les fabricants de caméras. La tendance actuelle est à leur implantation sur des systèmes informatiques compatibles IBM PC.

Des analyseurs de ligne industriels apparaissent également : un détecteur ponctuel et un balayage optomécanique unique permettent l'obtention d'une centaine de points par ligne à une fréquence allant jusqu'à $100 \mathrm{~Hz}$. Ces appareils sont initialement dédiés au contrôle IR de processus de fabrication (métallurgie, papeteries, contrôles de soudures,...). La chaîne d'acquisition et d'exploitation de leurs signaux utilise des logiciels élaborés également implantés sur microcalculateur.

Dans le domaine des pyromètres IR, l'introduction de fibres optiques permet de mesurer les flux rayonnés par des objets cachés ou difficilement observables directement. La mise en œuvre de fibres particulièrement transparentes dans l'IR est recherchée (fibre en verre fluoré, en verre au chalcogénures,...) m.

\subsection{Fibres optiques}

Les fibres optiques apportent un moyen efficace à la mesure des températures élevées, comme celles rencontrées dans les chambres de combustion, ou à la mesure des basses températures, comme celles rencontrées dans le domaine électrotechnique [35]. Ceci, grâce à leurs propriétés de tenue en température, d'isolation galvanique, d'insensibilité aux parasites et de faibles dimensions.

Quatre principes sont utilisés :

- la mesure par captation de rayonnement qui a débouché sur des réalisations commerciales (Photonetics par exemple);

- la mesure par photoluminescence qui a permis la commercialisation de trois produits (ASEA en Suède, LUXTRON aux USA et OMRON au Japon);

- la mesure par variation du guidage optique, limitée aux basses températures $\left(0-100^{\circ} \mathrm{C}\right)$;

- la mesure par variation d'absorption ou diffusion optiques qui a également débouché sur une commercialisation.

Il faut également indiquer que des perspectives intéressantes apparaissent en matière de possibilités de détection de points chauds le long d'une fibre optique.

\subsection{D.R.A.S.C. [36]}

La diffusion Raman anti-Stokes Coherente (DRASC) a été observée pour la première fois dans les liquides aromatiques par Maker et Terhune en 1965. Par la suite, elle avait été utilisée principalement à des fins spectroscopiques, notamment dans les solides. L'idée de l'employer pour effectuer des analyses chimiques non perturbantes est née à l'ONERA au début des années 70. Peu après, les premières mesures de concentration et de température dans les fiammes y étaient effectuées. Ces démonstrations de faisabilité sont à l'origine d'un développement extrêmement rapide de la méthode à l'ONERA mais aussi, à partir de 1975, dans les principaux laboratoires de recherche en combustion à travers le monde.

Aujourd'hui, le bilan est très riche. Des expériences variées et souvent extrêmement originales ont été menées à bien grâce à cette technique. En particulier, un nombre considérable de résultats pratiques a été obtenu sur des brûleurs et des foyers de recherche. Mais l'instrument, développé à l'origine pour le diagnostic thermodynamique des milieux en réaction, possède un champ d'applications bien plus vaste : étude de plasmas, de photochimie, de jets moléculaires, mais aussi analyse spectrale Raman des gaz purs qui se trouve grandement facilitée grâce aux gains considérables en résolution et vitesse d'acquisition permis par la DRASC. De fait, tous les milieux transparents (liquides et solides) peuvent être étudiés. Un instrument a été développé pour toutes ces applications. Cet instrument est fabriqué sous licence ONERA par la société SOPRA. Il est disponible commercialement, et trois exemplaires sont d'ores et déjà en service chez les clients français et étrangers.

\section{Mesures de hauteur de liquides}

En ce qui concerne les mesures de hauteur d'eau et de niveau, il existe une large gamme d'appareils basés sur des mesures de pression, ou utilisant des flotteurs, ou encore les ultrasons.

Récemment, Artémis a développé un capacimètre électronique en circuit intégré [31]. Cet appareil délivre un courant proportionnel au niveau d'un liquide le long d'une sonde, pour mesure et enregistrement à distance.

On peut ainsi suivre en amplitude et en fréquence les mouvements de la surface libre d'un liquide, mais également mesurer le niveau de la surface de séparation de deux liquides.

Différents modèles de sondes existent selon les applications qui sont très nombreuses :

- laboratoires d'hydraulique,

- centres de recherches,

- modèles physiques,

- plateformes d'essais,

- mesures dans l'industrie, sur les chantiers, dans les rivières et les ports,...

A titre d'exemple, le tableau XVII donne les caractéristiques d'une sonde de type $\mathrm{L}$, version laboratoire d'hydraulique.

Tableau XVII

\begin{tabular}{|l|l|}
\hline \multicolumn{2}{|c|}{ Cabactéristiques d'une sonde type L } \\
\hline Etendue de mesure & $\pm 10 \mathrm{~mA}$ sur charge $\leqslant 700 \Omega$ \\
& $\begin{array}{l}\text { pour } \pm 10 \mathrm{pF} \text { à } 10000 \mathrm{pF} \text { en } 5 \\
\text { gammes }\end{array}$ \\
Bandes passantes & $0-100 \mathrm{~Hz}, 0-10 \mathrm{~Hz}, 0-1 \mathrm{~Hz}, 0-0,1 \mathrm{~Hz}$ \\
Alimentation & $10 / 14 \mathrm{~V} \mathrm{C} \mathrm{C,} \mathrm{ou} \mathrm{:}$ \\
Sondes & $20 / 30 \mathrm{CC} \mathrm{CA}$ \\
& $\begin{array}{l}\text { diamètre depuis depuis } 0,2 \mathrm{~mm}, \\
\text { longueur ajustables, étalonnage } \\
\text { indépendant de la température et } \\
\text { de la salinité }\end{array}$ \\
\hline
\end{tabular}


D'autre part, la mesure des niveaux d'ergols dans les réservoirs de la fusée Ariane a nécessité la mise au point d'appareils utilisant les ultrasons. On mesure le temps de parcours aller et retour des ondes ultrasonores depuis un capteur jusqu'à la surface du liquide (figure 42).

Les améliorations récentes des performances des capteurs de pression, notamment à jauges de contraintes, ont permis de développer des limnimètres statiques dont la précision avoisine $10^{-3}$

La Division technique générale d'EDF a réalisé un tel dispositif à partir de capteurs de pression du commerce (Druck, Sedeme). Ils se présentent sous la forme d'un cylindre de dimensions réduites, contenant la partie sensible à jauges de contraintes associée à un convertisseur tension-courant.

La mise à l'air est obtenue au moyen d'un tube capillaire qui chemine dans le câble d'alimentation électrique.

Cet ensemble totalement étanche est fixé à l'extrémité d'un tube que l'on introduit dans un fourreau muni d'une crépine au niveau du point de mesure, permettant ainsi l'immersion et le positionnement précis du capteur. L'alimentation et l'exploitation de la mesure peuvent être réalisées à une grande distance du point de mesure, (jusqu'à $1 \mathrm{~km}$ ) et nécessitent seulement deux fils. La mesure est disponible aux bornes d'une résistance insérée dans le circuit d'alimentation.

La précision obtenue avec cet ensemble est de l'ordre de $1 \mathrm{~cm}$ sur $10 \mathrm{~m}$ d'eau [38].

Pour la mesure de niveau d'eau en usine hydraulique (plan d'eau amont et aval usine), EDF-DTG a développé un appareillage utilisant un capteur à ultrasons. Celui-ci, situé dans l'air au-dessus du plan d'eau et fixé au moyen d'une potence, mesure la distance à ce plan d'eau par la mesure du temps de parcours aller-retour de l'onde ultrasonore délivrée par le capteur, d'une part après réflexion sur le plan d'eau, d'autre part après réflexion sur un cible fixe. La précision obtenue par ce dispositif est de l'ordre de $\pm 5 \mathrm{~mm}$.

\section{Mesure de la qualité de l'eau}

Indépendamment de la nécessité du contrôle de la qualité de l'eau potable, la pollution des rivières et des mers par des eaux industrielles et des rejets a nécessité la mise au point de diverses méthodes que le tableau XVIII répertorie :

Tableau XVIII

\begin{tabular}{|cl|}
\hline Méthodes de mesure de la qualité de l'eau \\
\hline $7-1$ & Turbidité \\
$7-2$ & Analyse chimique \\
$7-3$ & Biomasse \\
\hline
\end{tabular}

\subsection{Turbidité}

La turbidité d'un liquide est due à la présence de petites particules dans ce liquide. De ce fait, elle ne peut être mesurée que par différentes méthodes [39], notamment par des procédés optiques.

Depuis le turbidimètre à bougie de Jackson, et les méthodes du fil de platine ou des gouttes de mastic, des progrès considérables ont été accomplis.

Le turbidimètre à diffusion lumineuse repose sur le principe suivant :

En envoyant un fin faisceau lumineux sur un flacon transparent contenant un liquide chargé de matières en suspension, on voit la turbidité du liquide quel que soit l'angle sous lequel on regarde ce flacon. En rencontrant une particule, la lumière incidente est diffusée dans toutes les directions et cette lumière diffusée est fonction du nombre de particules.

$\mathrm{Si}$ on analyse la lumière diffusée à $90^{\circ}$, on obtient un appareil appelé Nephelomètre.
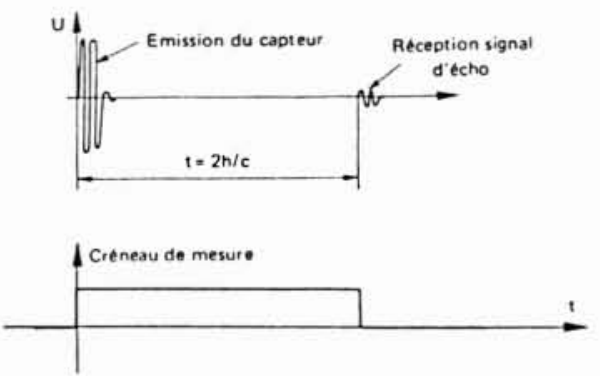

42. Mesure par ultrasons des niveaux d'ergols sur le lanceur Ariane.
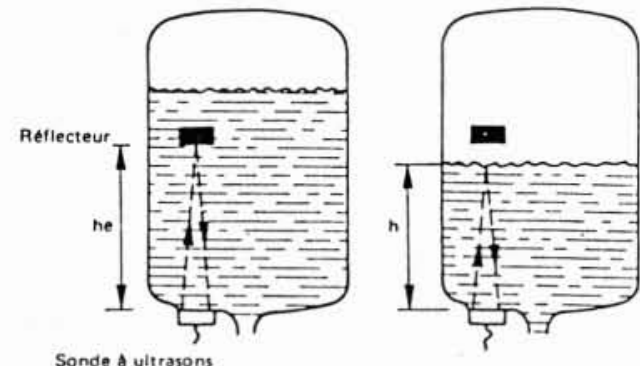


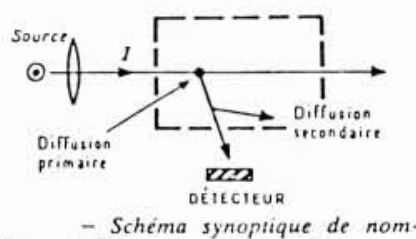

breux turbidimétres de laboratoire.

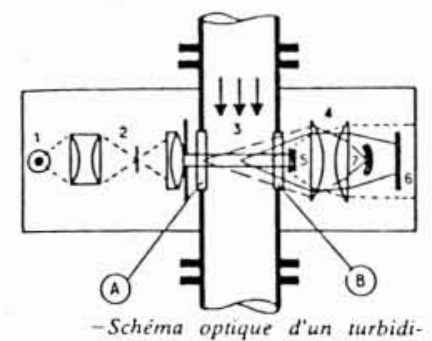

mètre " Monitek ".

1 source lumineuse ; 2 optique ; 3 fluide à analyser: 4 lentilles convergentes: 5 de. recteur du faisceau direci (références. 6 détecteur du foisceau diffuse sous $12^{\circ}$ (mesure). 7 corps noir (piege de lumière (mesure): 7 corps noir (piege de lumiere
parasite).

43. Schémas de principe de turbidimètres.

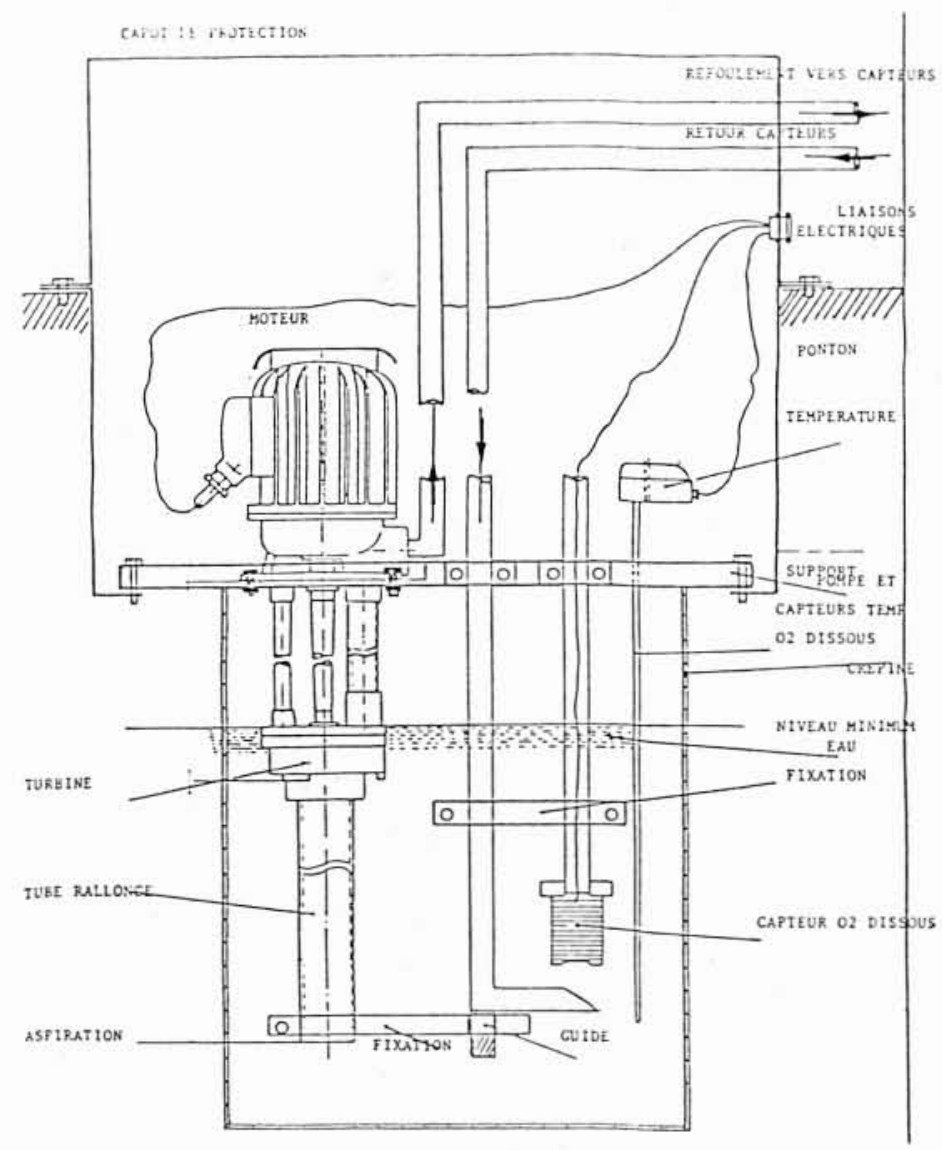

44. Station de contrôle des eaux de rivière. (Mesures de conductivité, oxygène dissous, pH et température). 
Mais selon les dimensions des particules (diamètre rapporté à la longueur d'onde de la source lumineuse), la distribution angulaire de la lumière diffusée varie notablement.

Pour les mesures en continu, de nombreux turbidimètres sont basés sur le principe indiqué sur la figure $43 a$. Ce système a toutefois l'inconvénient de ne pas tenir compte des dérives de la source lumineuse, de l'encrassement des fenêtres et de la diffusion secondaire. Pour corriger ces erreurs, des turbidimètres à double faisceau avec source lumineuse unique ont été réalisés (figure $43 \mathrm{~b}$ ).

Seule la lumière diffusée au centre est captée par le détecteur de mesure et la turbidité est donnée par le signal mesuré rapporté au signal référence.

D'autre part, les appareils sont munis de dispositifs spéciaux pour tenir compte de certains problèmes particuliers, tels que : bulles ou produits encrassants.

Il existe plusieurs unités de mesure de la turbidité. La plus couramment utilisée est le $\mathrm{mg}$ de $\mathrm{SiO}_{2}$ par litre d'eau ou ppm de silice.

Récemment, des appareils de principe différent sont apparus sur le marché. On peut citer notamment un dispositif original mesurant la turbidité totale (corps dissout et corps en suspension) avec une précision de l'ordre de $10^{-1} \mathrm{~g} / \mathrm{l}$.

Son principe consiste à faire circuler le fluide dans un conduit constituant un élément d'un diapason. On détecte alors la variation de la fréquence de résonance du système qui est directement liée à la turbidité totale.

Le tableau $X I X$ ci-après récapitule un certain nombre d'appareils de différents principes actuellement disponibles sur le marché.

Quant aux applications de ce type d'appareil, elles sont variées :

- surveillance des eaux potables,

- contrôle de la qualité de la bière,

- contrôle des huiles végétales,

- surveillance des vins,

- contrôle de l'eau d'alimentation des chaudières,

- contrôle de l'eau des tours de réfrigération,

- surveillance de l'eau des papeteries,

- surveillance des eaux résiduelles urbaines,

- contrôle du kérosène,

- etc.

Tableau XIX. - Liste des turbidimètres disponibles sur le marché

\begin{tabular}{|l|l|}
\hline \multicolumn{1}{|c|}{ Fabricant } & \multicolumn{1}{|c|}{ Principe } \\
\hline Degrémont & Diffusion de la lumière \\
Thermanalyse & $\begin{array}{l}\text { Mesure de densité par fréquence de } \\
\text { Instrulab }\end{array}$ \\
C.E.M. & Diffusion de la lumière \\
Kontron & Absorption de lumière \\
Soptel & Diffusion de la lumière \\
Safas & Spectrophotomètre \\
Wilson & Diffusion de lumière \\
Walton & \\
Automation & Opacité \\
Industrie & \\
Industel & Opacité \\
Fabre & Absorption de lumière \\
Bestobell & \\
Mobrey & \\
C.E.A. & Diffusion des photons \\
\hline
\end{tabular}

\subsection{Eaux de rivière}

Pour les besoins d'EDF, des stations de contrôle ont été réalisées et équipent certaines centrales nucléaires [40]. Elles sont constituées de trois chaines de mesure de conductivité à oxygène dissous et $\mathrm{pH}$ et d'une chaîne de mesure de température.

Le principe en est le suivant : les quatre capteurs et la pompe de circulation sont situés sur un ponton flottant (figure 44); les capteurs de conductivité et de $\mathrm{pH}$ sont placés dans une chambre à circulation fixée dans un coffret étanche solidaire du ponton; les capteurs de température et d'oxygène dissous sont situés dans un puits de mesure faisant office de crépine.

Les signaux de mesure sont transférés par câbles dans un local où se trouve une armoire comportant les transmetteurs, les indicateurs et les enregistreurs.

La conductivité est mesurée à partir de la résistance du liquide compris entre deux électrodes en graphite, alimentées par signaux carrés à $300 \mathrm{~Hz}$. L'échelle de mesure est de 0 à $1000 \mu \mathrm{s} / \mathrm{cm}$.

Le $\mathrm{pH}$ est mesuré à l'aide de deux électrodes spéciales : l'une en verre possède un bulbe épais, l'autre, de référence, est rechargeable avec du $\mathrm{KCl}$ semi solide. La jonction électrolytique est constituée d'une pastille de bois dur qui permet seulement une diffusion ionique. Le signal à haute impédance et bas niveau est amplifié et envoyé au transmetteur.

L'échelle de mesure est de 6-11 ou 5-10 selon les sites.

La mesure de température est effectuée à l'aide d'une résistance de platine de $100 \Omega$ à $0{ }^{\circ} \mathrm{C}$, montée dans une branche d'un pont.

L'échelle de mesure est de $0-50{ }^{\circ} \mathrm{C}$.

La mesure de l'oxygène dissous est une mesure polarographique; l'oxygène diffuse à travers une membrane en téflon, et passe dans une' cellule de mesure composée d'une cathode en or et d'une anode en argent baignant dans un électrolyte ( $\mathrm{KCl} 2 \mathrm{M})$.

L'échelle de mesure est de $0-20 \mathrm{mg} / \mathrm{l}$.

L'ensemble des mesures est enregistré sur enregistreurs potentiométriques mais également mis en mémoire pour traitement ultérieur.

\subsection{Biomasse}

La biomasse du plancton végétal, tant en mer que dans les eaux douces et saumâtres, est généralement mesurée à partir de la concentration en chlorophylle a.

Un indice de dégradation a été proposé qui permet :

- de mettre en évidence la dégradation partielle de la chlorophylle a [41],

- de suivre l'évolution du phytoplancton dans le temps et dans l'espace.

La biomasse algale et bactérienne des cours d'eau, comme celle des boues des stations d'épuration, peut être estimée par extraction et dosage de l'ATP (adenosine triphosphate) constituant intracellulaire universel de toutes les cellules vivantes [42]. Récemment, l'Institut de recherches hydrologiques de Nancy a mis au point une méthode permettant de mesurer séparément l'ATP algal de l'ATP bactérien par filtration différentielle. 


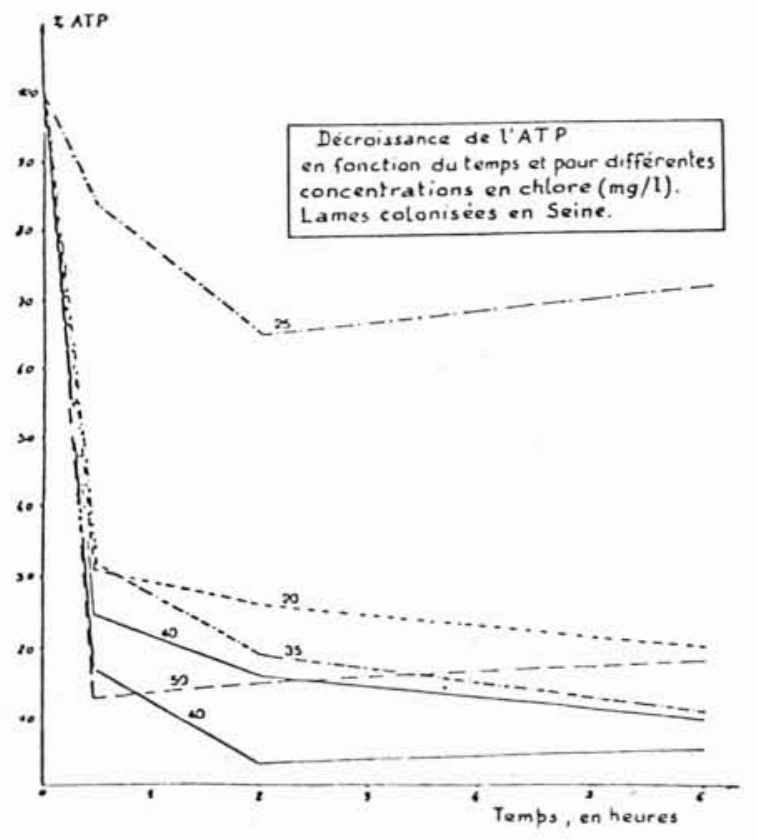

45. Décroissance de l'ATP en fonction du temps.
Cette méthode globale de mesure de biomasse a été expérimentée par I'EDF de façon très satisfaisante, et la figure $\mathbf{4 5}$ montre un exemple de résultat de détermination de l'efficacité du traitement biocide appliqué dans les réfrigérants atmosphériques.

Des supports artificiels ont été placés dans l'eau d'un circuit équipé de réfrigérants atmosphériques. La mesure de l'ATP des dépôts recueillis permet de contrôler l'action du chlore sur les salissures biologiques qui encrassent les canalisations et le réfrigérant. Comme le montre la figure 45, la décroissance du taux d'ATP est maximale au bout de deux heures et correspond au maximum d'efficacité du traitement.

\section{Diphasique}

Les mesures effectuées dans les écoulements diphasiques étant particulières ou regroupant plusieurs mesures simultanées comme la taille et la vitesse de gouttes, il nous a semblé préférable de rassembler toutes ces mesures dans ce chapitre, même si l'on en retrouve quelques une précédemment exposées.

Quant au classement choisi, il est indiqué dans le tableau $X X$.

Tableau $\mathrm{XX}$

\begin{tabular}{|ll|}
\hline \multicolumn{2}{|c|}{ Mesures en diphasique } \\
\hline $8-1$ & Granulométrie \\
$8-2$ & Vitesse de bulles \\
$8-3$ & Titre \\
$8-4$ & Mesures atmosphériques \\
\hline
\end{tabular}

\subsection{Granulométrie}

Une étude bibliographique a été effectuée sur le sujet il $\mathrm{y}$ a quelques années à partir d'articles de la bibliothèque du CNRS des années 1976-1977, puis très récemment par P. Hamelin [43].

8.1.1. Parmi les méthodes optiques utilisées pour déterminer la taille des particules liquides ou solides que l'on trouve dans les brouillards, aérosols, etc. l'holographie est un moyen.

Une telle technique a été mise au point à EDF [44], qui permet de reconstituer en trois dimensions, l'image d'un objet à partir d'un seul enregistrement.

Dans le cas de particules comme des gouttelettes d'eau en suspension dans un milieu homogène, on utilise le montage schématisé sur la figure 46; tout d'abord enregistrement de l'hologramme (figure $46 a$ ), puis restitution de l'image (figure $46 \mathrm{~b}$ ) à partir de laquelle on obtient la dimension des gouttelettes.

Cette technique permet, en même temps, de déterminer la vitesse. Il suffit d'holographier à deux instants différents sur une même plaque; ce qui est très facile à l'aide d'un laser pulsé à rubis qui permet deux impulsions rapprochées de 2,5 à $50 \mu \mathrm{s}$. 
Une telle méthode a de nombreux avantages :

- restitution dans l'espace,

- mesure directe,

- simplicité optique,

- haute résolution,

- analyse de volumes importants,

- vitesses relativement élevées possibles.

Il y a toutefois quelques limitations et conditions à respecter concernant l'éloignement, le bruit de fond, la densité des particules, la qualité des optiques et la relative lenteur des dépouillements.

La méthode a été employée avec succès dans des conditions variées :

- jet de lance à incendie,

- arrachement d'un film d'eau sur un profil,

- brouillard à pulvérisation mécanique,

- brouillard à pulvérisation pneumatique.

8.1.2. Une autre méthode optique de mesure de la taille de gouttes, repose sur l'atténuation d'intensité subie par un fasceau lumineux traversant un brouillard de gouttelettes. dans le cas d'une population dispersée, l'atténuation est donnée par:

$$
\frac{I}{I_{0}}=\exp \left[-\sum n r i^{2} n(r i) K(r i, \lambda) L\right]
$$

où $n(r i)$ est le nombre de gouttes de rayon $r$ et $K(r i, d)$ $=K(2 \pi r i / \lambda)$ est le coefficient d'absorption de Penndorf.

Pour calculer la granulométrie cherchée, l'expression ci-dessus ne suffit pas. Il faut d'autres informations que l'on obtient en utilisant plusieurs longueurs d'ondes.

La précision obtenue est d'autant meilleure que le nombre de mesures, donc de longueurs d'ondes utilisées, est important.

8.1.3. Une troisième méthode optique est basée sur la diffraction de la lumière. On étudie la diffraction d'une onde plane monochromatique rencontrant une sphère isotrope et homogène. La figure de diffraction obtenue dans le plan focal d'une lentille, (c'est-à-dire la transformée de Fourier de la sphère), ne dépend que de la géométrie de l'obstacle et non de sa nature. On obtient une figure composée d'anneaux alternativement sombres et clairs, d'intensité décroissante à partir du centre, appelée fonction d'Airy.

En principe, cette méthode n'est valable que pour des particules de dimensions supérieures de 5 à 10 fois la longueur d'onde de la lumière qui les éclaire.

Sinon, il faut utiliser la théorie très générale de Mie.

En pratique, il existe deux types de méthodes d'application de cette technique :

- méthode de comptage de particules une à une,

- méthode d'ensemble.

La première est adaptée aux faibles concentrations, mais la mesure est longue à effectuer, un étalonnage précis est nécessaire et de plus la connaissance de la vitesse est indispensable.

La seconde peut être adaptée en temps réel, est simple d'emploi, commode d'étalonnage et peut être utilisée dans le cas de fortes concentrations. Toutefois le traitement mathématique est compliqué et l'obtention d'une concen- tration en valeur absolue, peu précise (cf. granulomètre Cilas).

8.1.4. Une quatrième méthode optique repose sur l'analyse du spectre en fréquence obtenu par diffraction de particules. La mesure de la puissance diffusée en fonction de la fréquence, aboutit, après calcul, à la taille moyenne et à la densité de particules diffractantes.

Une telle méthode donne des résultats convenables si la distribution des tailles ne dépasse pas $30 \%$ de la taille moyenne. Le temps de mesure est de 1 minute. Enfin, la limite inférieure expérimentale pour le diamètre est de 0,2 $\mu \mathrm{m}$.

8.1.5. Une cinquième méthode optique consiste à utiliser l'anémométrie laser. L'idée de base est d'utiliser la profondeur de modulation du signal Doppler alternatif et de la relier à la taille de la particule. Elle suppose, d'autre part, qu'une seule particule traverser le volume de mesure, c'est-à-dire que la concentration est limitée [43], [45]. Cette technique est en plein développement avec de nombreuses variantes.

8.1.6. Une dernière méthode optique ou méthode d'ombre consiste à diriger un laser sur un phototransistor, soit directement, soit par l'intermédiaire de filtres optiques.

L'intensité est donnée par: $I_{2}=I_{0} \exp \left(-2 r^{2} / r_{0}^{2}\right)$.

Un très petit diaphragme est placé contre le phototransistor pour ne recevoir que la portion du faisceau telle que

$$
r<r_{0}
$$

Quand le phototransistor reçoit tout le faisceau laser, ainsi délimité, on règle sa sortie juste au-dessous de la saturation.

L'arrivée d'une particule, diffractante ou non, bloque pratiquement le rayon incident pourvu que le récepteur soit assez loin de la particule et que le diamètre soit beaucoup plus grand que la longueur d'onde.

Il faut que le diamètre du faisceau soit plus grand que celui de la particule pour qu'il n'y ait pas saturation du signal. La technique de mesure est rapide.

8.1.7. A côté de ces méthodes optiques, il existe une méthode acoustique pour la détermination de la taille de bulles. Cette méthode est basée sur l'atténuation et la dispersion d'une onde acoustique de fréquence comprise entre $20 \mathrm{kHz}$ et $200 \mathrm{kHz}$.

Un train d'onde sinusoïdal est émis pendant $0,5 \mathrm{~ms}$, puis on recueille les échos sur oscilloscope.

La méthode est valable pour des concentrations limitées et des mesures ont été faites pour des valeurs de rayons de bulles compris entre $30 \mu \mathrm{m}$ et $800 \mu \mathrm{m}$.

Le plus sérieux inconvénient est le fait que les particules ne passent pas forcément au centre du faisceau, c'est-à-dire que l'on mesure en général des tailles trop petites.

8.1.8. Enfin, nous signalerons une dernière méthode, dite calorimétrique. On utilise une sonde calorimétrique électrique qui, (pour des mesures en vapeur humide), assèche la vapeur; connaissant la puissance dépensée, on peut théoriquement remonter jusqu'au titre.

Cette technique a été utilisée par EDF pour diverses expérimentations [46]. 


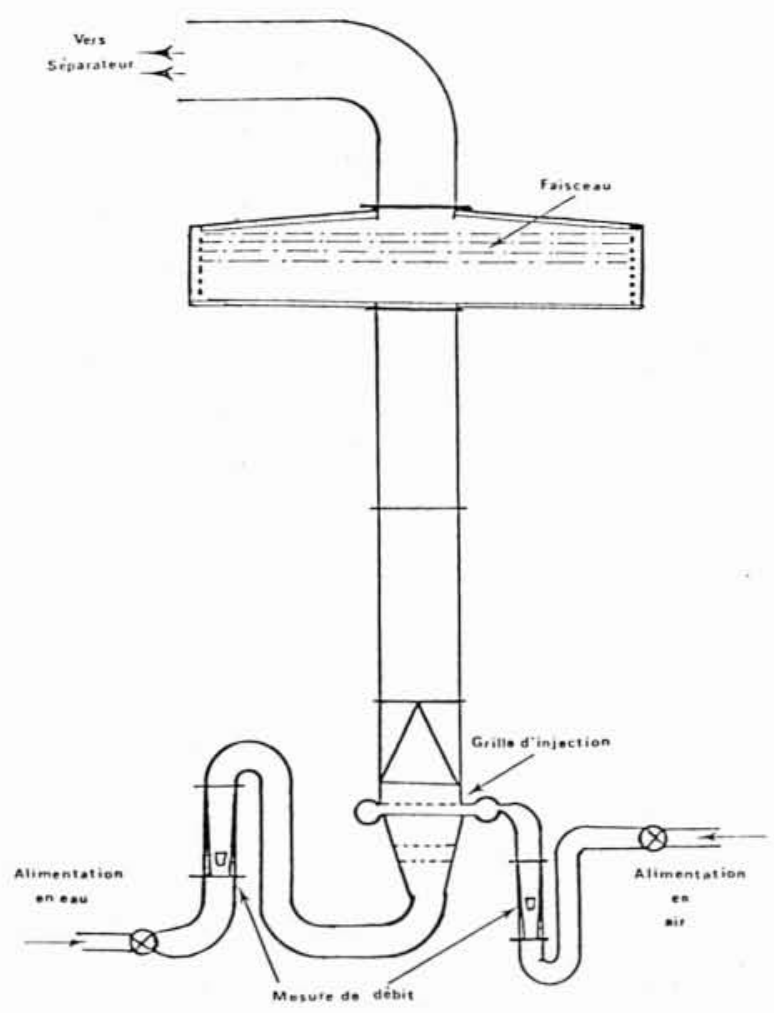

47. Schéma de la boucle d'essai.

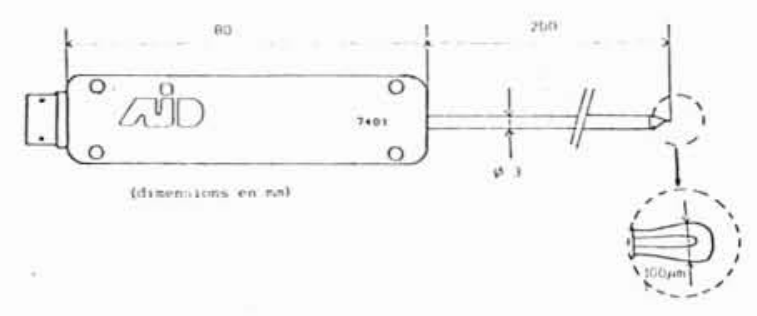

48. Schéma de la sonde à fibre optique.
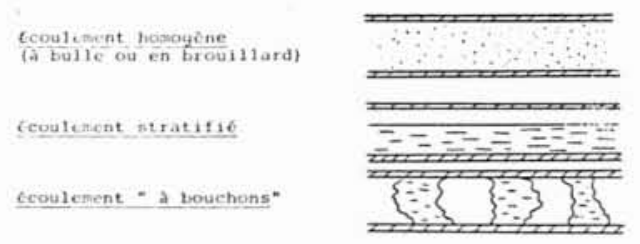

50. Formes possibles d'un écoulement diphasique.

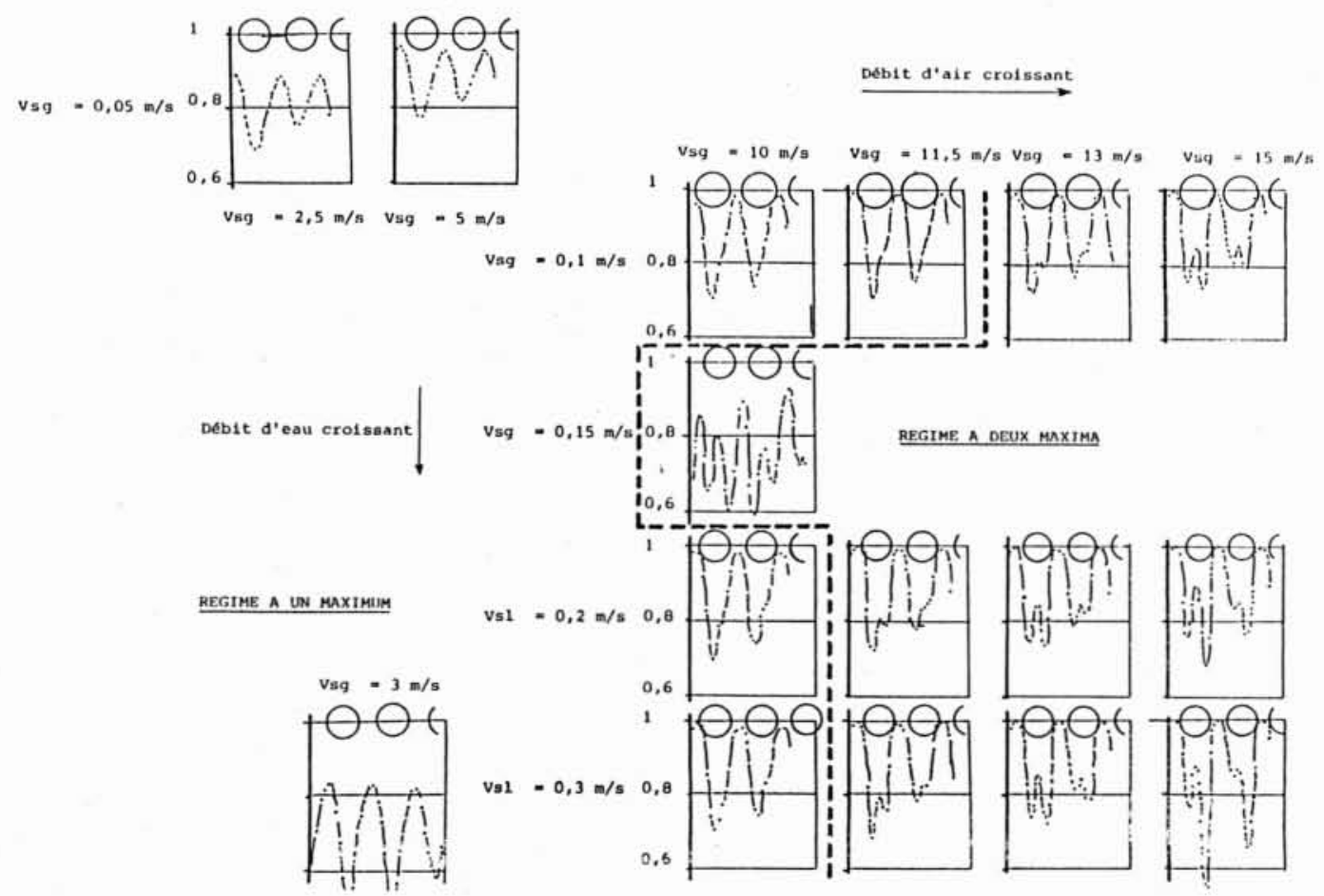

49. Evolution du taux de présence en fonction des débits d'air et d'eau. 


\subsection{Vitesse de bulles}

Comme application de la vélocimétrie laser aux écoulements diphasiques, la vitesse de bulles d'hydrogène contenues, à concentration élevée, dans un écoulement de potassium, a été déterminée dans des expériences conduites par EDF [47]. Le diamètre des particules variait de 4 à $120 \mu \mathrm{m}$, les concentrations atteignaient $10^{8} / \mathrm{cm}^{3}$ pour les petites particules tandis que des vitesses jusqu'au $\mathrm{mm} / \mathrm{s}$ ont été mesurées.

Plus récemment un développement de la méthode a été entrepris, également à EDF [43].

Cette même technique a aussi été utilisée pour mesurer la vitesse de bulles d'air de grande taille (diamètre $\sim 5 \mathrm{~mm}$ ) dans un écoulement d'eau [48]. Il s'agit de l'application de la méthode interférentielle en diffusion avant. Ce dernier procédé améliore le rapport signal/bruit, mais dans le cas présent, la lumière réémise par les bulles est maximale vers l'avant.

La chaîne d'acquisition du banc d'essais permet de stocker l'information "fréquence " transmise par le compteur et d'en effectuer le traitement instantané ou différé.

Des procédures particulières de traitement du signal ont été mises au point pour ce type d'écoulement à deux phases.

De bons recoupements ont également été obtenus avec des mesures par film chaud.

\subsection{Titre}

\subsubsection{Taux de présence}

La mesure du taux de présence dans un faisceau tubulaire placé dans un écoulement diphasique a été entreprise à EDF à l'aide d'une sonde à fibre optique [49]. Cette mesure du taux de présence d'une phase par rapport à l'autre donne accès à la vitesse de glissement entre phase.

De telles mesures ont été effectuées sur une boucle d'essai représentée sur la figure 47 à l'aide d'une sonde à fibre optique schématisée sur la figure 48.

Le principe de cette sonde repose sur la propriété d'un rayon lumineux de se réfléchir sur un dioptre, dans certaines conditions d'incidence et de valeur des indices des deux milieux.

La sonde est constituée d'une fibre optique formant une boucle. A une extrémité, une lumière est émise. Lorsque le milieu est de l'air, la lumière n'est pas réfractée et suit donc la boucle pour être détectée à l'autre extrémité.

La tension délivrée est 5 volts dans l'air et 0 volt dans l'eau.

Cette sonde a donc été appliquée à des mesures sur le montage indiqué pour différents couples de débit, mesures effectuées dans le canal amont et dans le faisceau de tubes.

On a ainsi pu mettre en évidence :

- une organisation remarquable entre les deux phases de l'écoulement, faisant apparaître deux régimes possibles dans les plages de débit considérées (figure 49).

- que la présence du faisceau ne modifie par les grandeurs intégrées sur une section.

\subsubsection{Taux de vide}

Comme le montre la figure 50, un écoulement diphasique peut se présenter sous trois formes limites :

- écoulement homogène,

- écoulement stratifié,

- écoulement "à bouchons".

Il est évidemment intéressant de savoir dans quel cas l'on se trouve. Il existe une méthode de mesure classique : " la gammagraphie ", (mesure par absorption d'un rayonnement $\gamma$ ), mais elle est sensible aux fluctuations spatiales et temporelles du taux de vide.

Une technique nouvelle de gammagraphie à " 2 énergies " a alors été développée à EDF [50], et utilisée sur un banc d'essais de pompe en diphasique représenté sur la figure 51 .

Les essais se faisant par paliers de 15 à $20 \mathrm{sec}$., un programme fait alors la moyenne des pressions, des comptages et des températures. On utilise ensuite ces moyennes pour évaluer les taux de vide linéiques $100 \mathrm{KeV}$ et $40 \mathrm{KeV}$, ainsi que le taux de vide réel.

On a pu ainsi mettre en évidence des régimes de fonctionnement avec fluctuations importantes (écoulement du type à bouchons), et des régimes très homogènes.

Un autre moyen existe pour mesurer ce taux de vide [51]; il s'agit d'une méthode par dilution d'un traceur radioactif non volatil. On injecte en continu ce traceur en amont de la section de mesure et lorsqu'il est convenablement mélangé avec le fluide, une première mesure directe d'activité volumique est effectuée avant que la phase gazeuse n'apparaisse; c'est une mesure de référence. On mesure ensuite l'activité volumique du fluide dans la section mesure, ce qui permet d'obtenir le taux de vide.

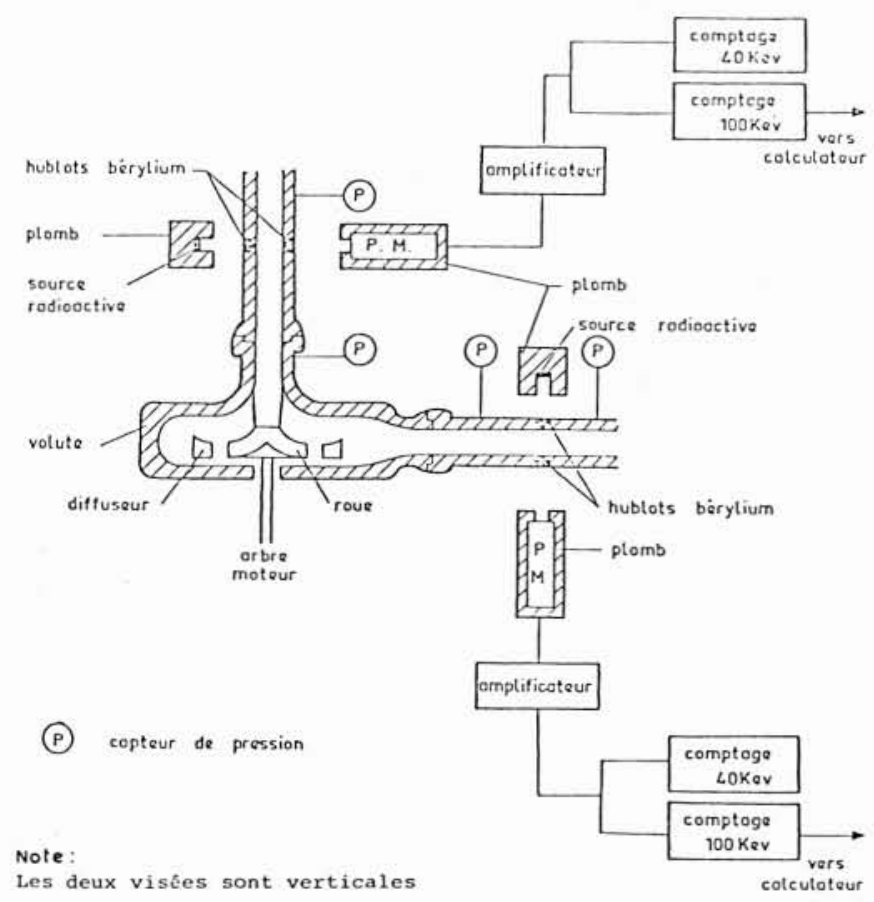

51. Banc d'essais pour mesures de gammagraphie à " 2 énergies ". 
Les deux méthodes indiquées ont bien sûr des avantages et des inconvénients et dans la mesure du possible, il est souhaitable d'utiliser les deux comparativement.

$\mathrm{Si}$ une seule méthode est possible à mettre en œuvre, la gammagraphie pourra être utilisée pour des taux de vide inférieurs à $90 \%$; la méthode par dilution sera par contre préférée dans le cas de taux de vide supérieurs à $90 \%$ (dans l'hypothèse d'un glissement négligeable).

Une méthode de reconstruction de la distribution spatiale du taux de vide dans une conduite par tomographie $\gamma$ est en cours d'étude à EDF.

Il faut également citer les sondes optiques diphasiques développées par AID à Grenoble et Photonétics à Marly-le-Roi.

Le principe de mesure est fondé sur les variations du coefficient de réflexion de la lumière à l'extrémité d'une fibre optique plongée dans l'écoulement. L'extrémité d'une fibre immergée dans un liquide réfléchit moins la lumière que dans un gaz. Cet effet permet d'identifier la phase du milieu situé en bout de fibre, de détecter le passage d'une bulle de vapeur, de déterminer la proportion de bulles présentes dans la veine liquide passant à proximité de la fibre. En combinant les données de plusieurs sondes, on peut remonter au taux de vide global de l'écoulement.

\subsection{Mesures atmosphériques}

Les rejets de chaleur des grandes centrales de production d'électricité d'origine thermique et des concentrations de consommation d'énergie ont des répercussions atmosphériques qu'il importe de prévoir et d'identifier. D'où le besoin de mesurer verticalement et horizontalement certains paramètres : température, humidité, teneur en eau, granulométrie des gouttes, vitesse de l'air, rayonnement solaire.

Les matériels utilisés pour de telles mesures sont assez classiques, comme l'indique le tableau $X X I$ ci-après :

Tableau XXI. - Paramètres mesures et caractéristiques des matériels correspondants

\begin{tabular}{|c|c|c|c|}
\hline & Capteur & Paramètre mesuré & $\begin{array}{c}\text { Gamme } \\
\text { des mesures }\end{array}$ \\
\hline \multirow{2}{*}{ Température } & Rosemount & $\begin{array}{l}\text { Température } \\
\text { (référence) }\end{array}$ & $\pm 40^{\circ} \mathrm{C}$ \\
\hline & Sonde rapide INAG & $\begin{array}{c}\text { Température } \\
\text { (Fluctuations rapides) }\end{array}$ & $\pm 2{ }^{\circ} \mathrm{C}$ \\
\hline \multirow{3}{*}{$\begin{array}{l}\text { Teneur en } \\
\text { eau/vapeur }\end{array}$} & Cambridge & $\begin{array}{l}\text { Point de rosée } \\
\text { (référence) }\end{array}$ & $\pm 50^{\circ} \mathrm{C}$ \\
\hline & Bendix (hygristor) & Humidité relative & $5-95 \%$ \\
\hline & Lyman $\alpha$ & $\begin{array}{l}\text { Teneur en eau/vapeur } \\
\text { (Fluctuations rapides) }\end{array}$ & $0-20 \mathrm{~g} / \mathrm{m}^{3}$ \\
\hline $\begin{array}{l}\text { Teneur en } \\
\text { eau totale }\end{array}$ & $\begin{array}{c}\text { Lyman } \alpha \\
\text { par évaporation } \\
\text { de I'eau liquide } \\
\text { (système INAG) }\end{array}$ & $\begin{array}{l}\text { Teneur en eau totale } \\
\text { (vapeur + liquide) }\end{array}$ & $0-20 \mathrm{~g} / \mathrm{m}^{3}$ \\
\hline $\begin{array}{l}\text { Teneur en } \\
\text { eau liquide }\end{array}$ & Johnson Williams & Eau liquide & $0-6 \mathrm{~g} / \mathrm{m}^{3}$ \\
\hline $\begin{array}{c}\text { Granulométrie } \\
\text { des } \\
\text { Gouttes }\end{array}$ & $\begin{array}{l}\text { Knollenberg } \\
\text { FSSP } 100 \\
\text { OAP } 200 \times\end{array}$ & $\begin{array}{l}\text { Comptage } \\
\text { des } \\
\text { gouttes } \\
\text { par } \\
\text { taille }\end{array}$ & $\begin{array}{c}15 \text { classes } \\
\text { par gamme } \\
0,5-8 \mu \mathrm{m} \\
1-16 \mu \mathrm{m} \\
2-32 \mu \mathrm{m} \\
2-47 \mu \mathrm{m} \\
20-300 \mu \mathrm{m}\end{array}$ \\
\hline \multirow{4}{*}{$\begin{array}{c}\text { Composantes } \\
\text { moyennes } \\
\text { de la } \\
\text { vitesse } \\
\text { de l'air }\end{array}$} & $\begin{array}{l}\text { Centrale à inertie } \\
\text { SAGEM MGC } 30\end{array}$ & $\begin{array}{l}\text { Angle d'attitude } \\
\text { (roulis et tangage) }\end{array}$ & $\begin{array}{l} \pm 15^{\circ} \\
\pm 45^{\circ}\end{array}$ \\
\hline & Girouettes & $\begin{array}{c}\text { Angle d'incidence } \\
\text { du vent relatif }\end{array}$ & $\pm 20^{\circ}$ \\
\hline & $\begin{array}{c}\text { Crouzet } \\
\text { Schlumberger }\end{array}$ & $\begin{array}{c}\text { Pression statique } \\
\text { Pression différentielle }\end{array}$ & $\begin{array}{l}0-1100 \mathrm{mb} \\
0-\quad 50 \mathrm{mb}\end{array}$ \\
\hline & Radar Doppler & vitesse/sol & 0. $500 \mathrm{~m} / \mathrm{s}$ \\
\hline $\begin{array}{c}\text { Fluctuation de la } \\
\text { vitesse de l'air }\end{array}$ & Fil chaud INAG & $\begin{array}{c}\text { Fluctuations rapides } \\
\text { de la vitesse }\end{array}$ & $45-100 \mathrm{~m} / \mathrm{s}$ \\
\hline Rayonnement & $\begin{array}{c}\text { Pyranomètres } \\
\text { et } \\
\text { Radiomètres }\end{array}$ & $\begin{array}{l}\text { Flux radiatifs } \\
\text { direct et } \\
\text { réfléchi }\end{array}$ & $\begin{array}{l}0-1500 \mathrm{w} / \mathrm{m}^{2} \\
0.750 \mathrm{w} / \mathrm{m}^{2}\end{array}$ \\
\hline
\end{tabular}


Un autre moyen d'étude des panaches des réfrigérants atmosphériques est le "Lidar" [52]. Cet appareil comprend un laser de puissance qui émet des impulsions lumineuses de $20 \mathrm{~ns}$ à une cadence maximale de $20 \mathrm{~Hz}$. Un télescope de $30 \mathrm{~cm}$ de diamètre recueille les photons diffusés par les aérosols ou les gouttes d'eau présents sur le trajet du faisceau laser. Un analyseur rapide échantillone et numérise toutes les $50 \mathrm{~ns}$ le signal électrique issu du photodétecteur. Ces informations numériques sont enregistrées sur disque souple par l'intermédiaire d'un minicalculateur qui prend en charge le déroulement complet des cycles de mesure : mouvements de la platine site-azimut et visualisation des résultats.

La cartographie d'un panache peut être effectuée en quelques dizaines de minutes.

La figure 52 montre une vue de cet appareil.

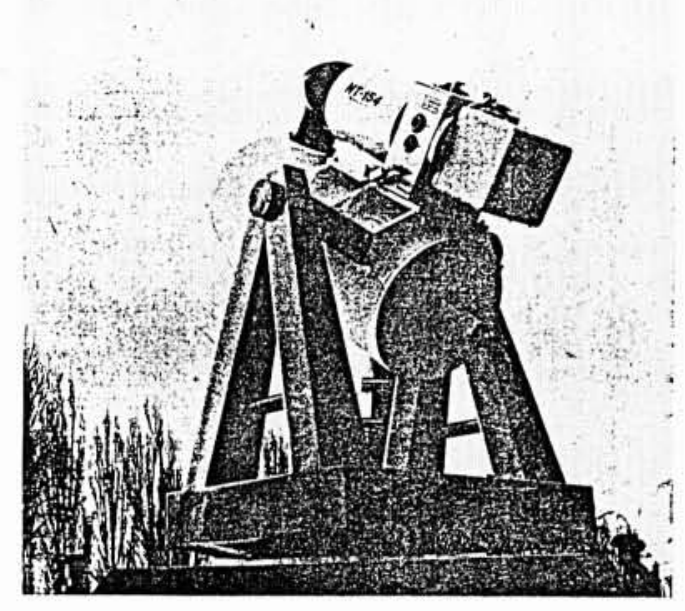

52. Vue du Lidar.

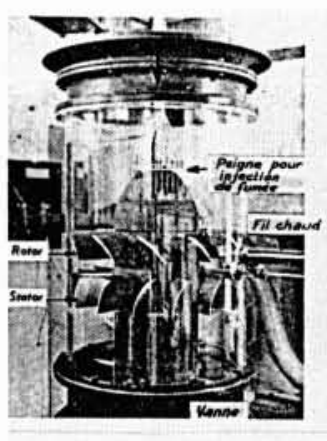

Vue du compresseur

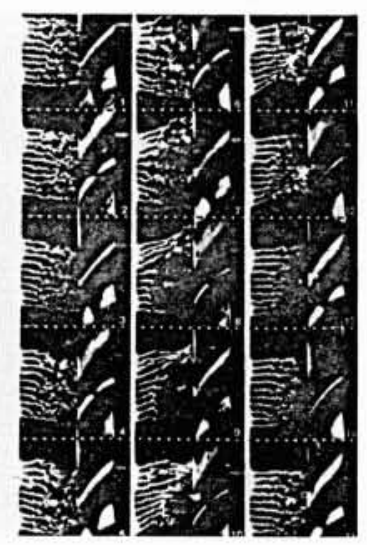

53. Visualisation par filets de fumée. (Régime de décollement tournant).

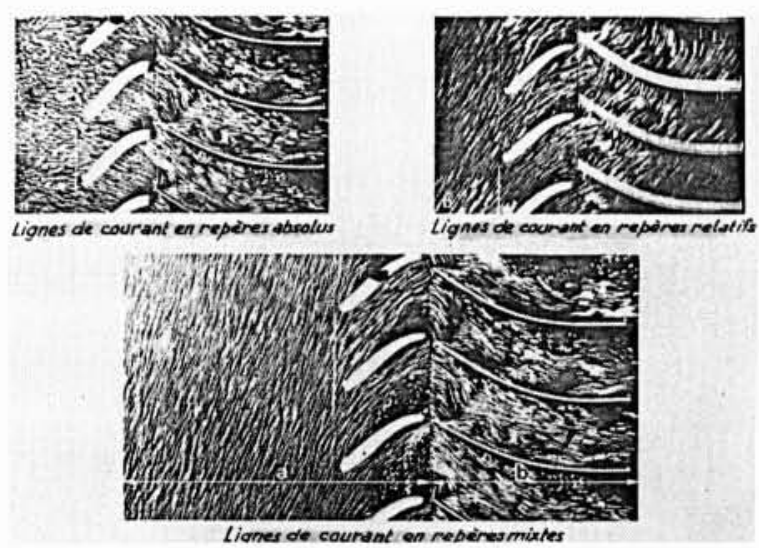

54. Visualisation, par fines particules, de l'écoulement dans une grille plane mobile. (Analogie hydraulique). minces et plus intenses que les tranches classiques obtenues à partir d'une lampe et d'un système optique. 


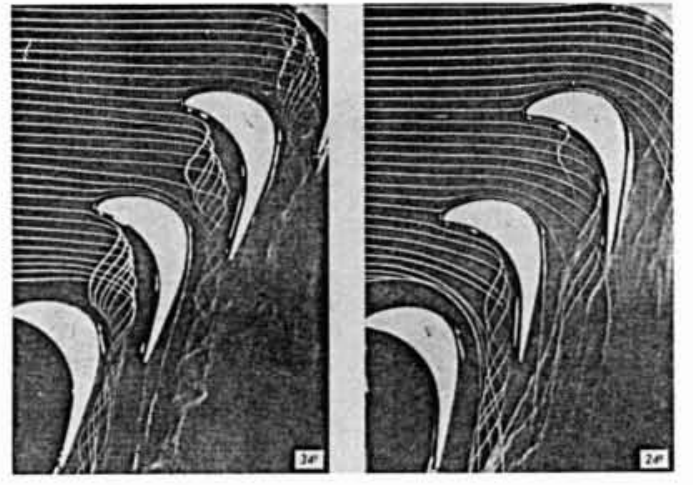

55. Visualisation, par filets colorés, des écoulements secondaires dans une grille fixe d'aubes de turbine (analogie hydraulique).

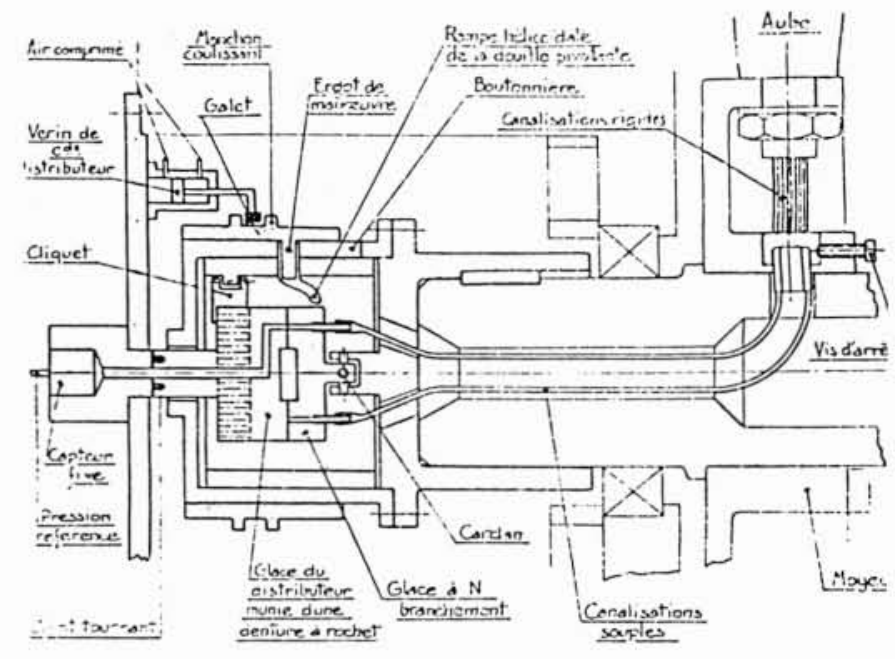

58. Turbocommutateur de pression.

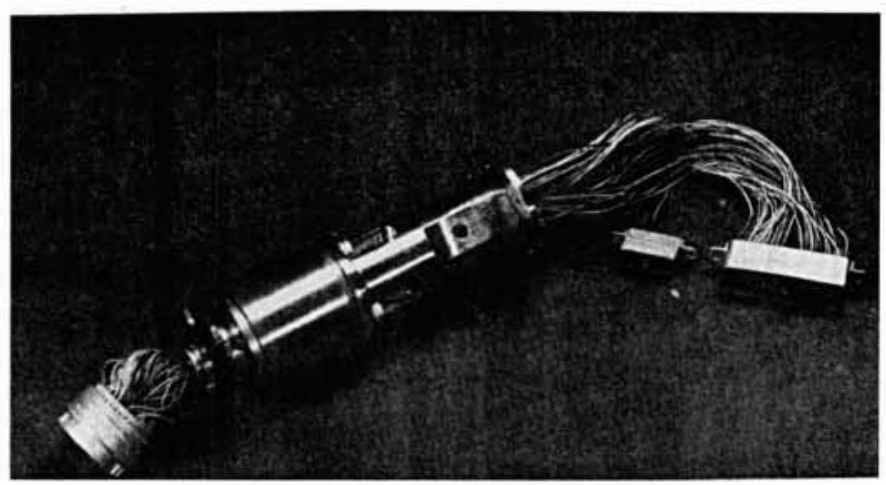

59. Exemple de contacteur à balais.

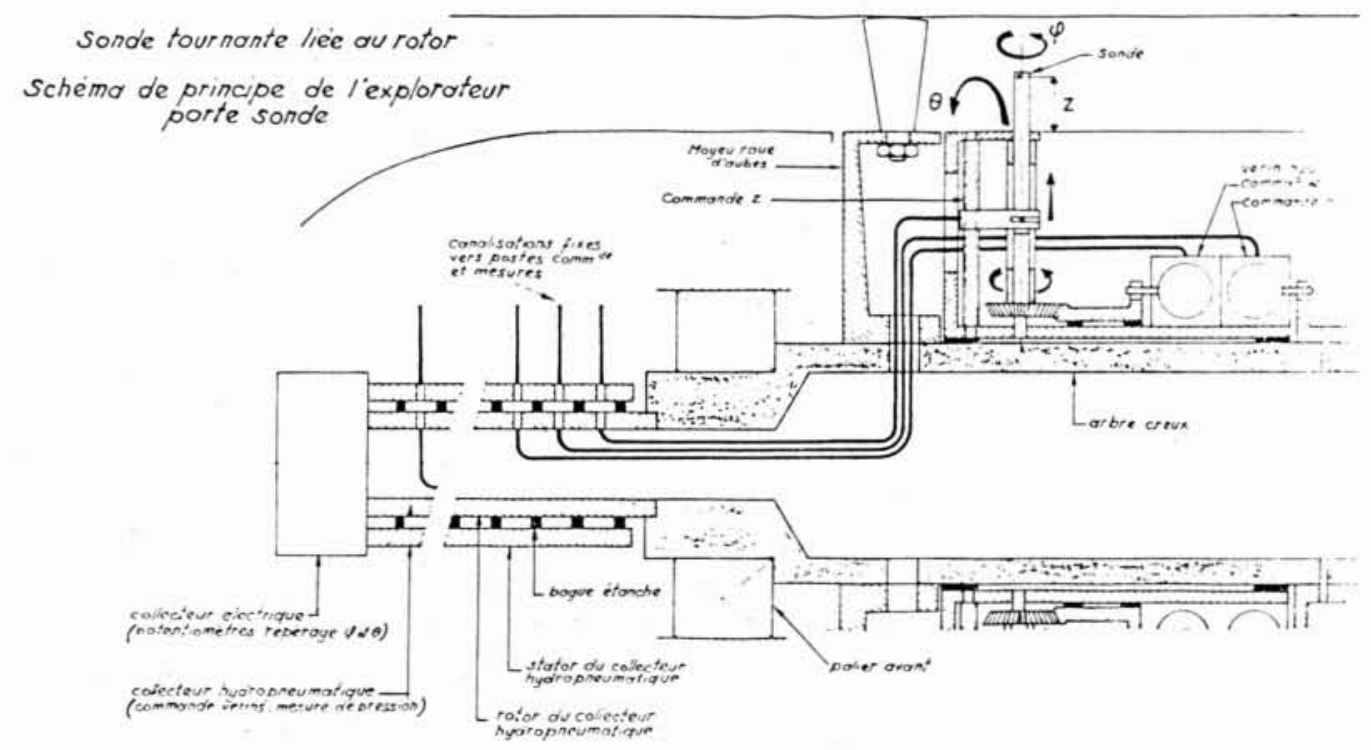

57. Dispositif explorateur pour sonde tournante. 

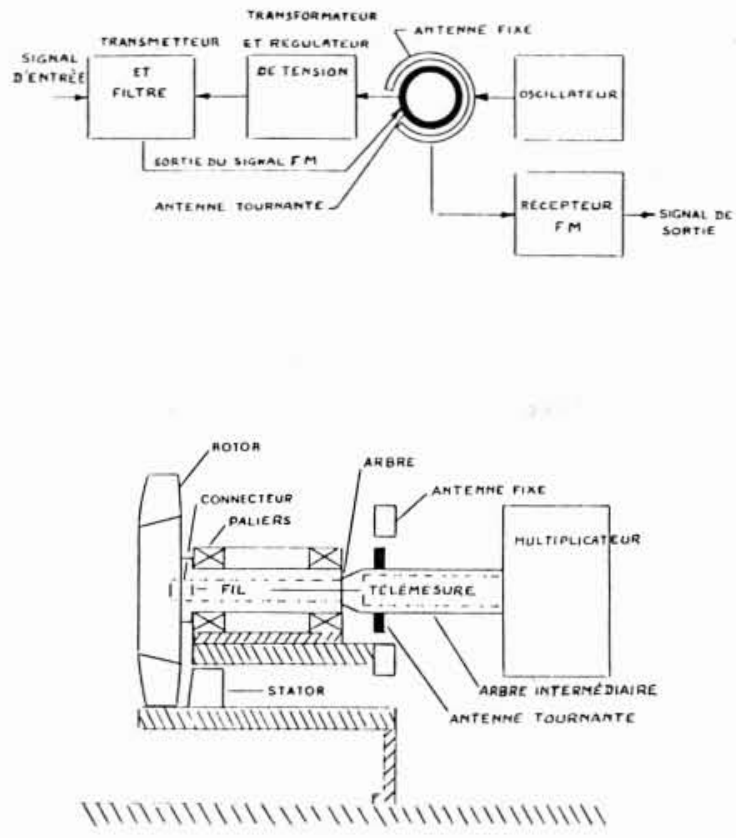

60. Schéma de principe d'un dispositif de télémesure.
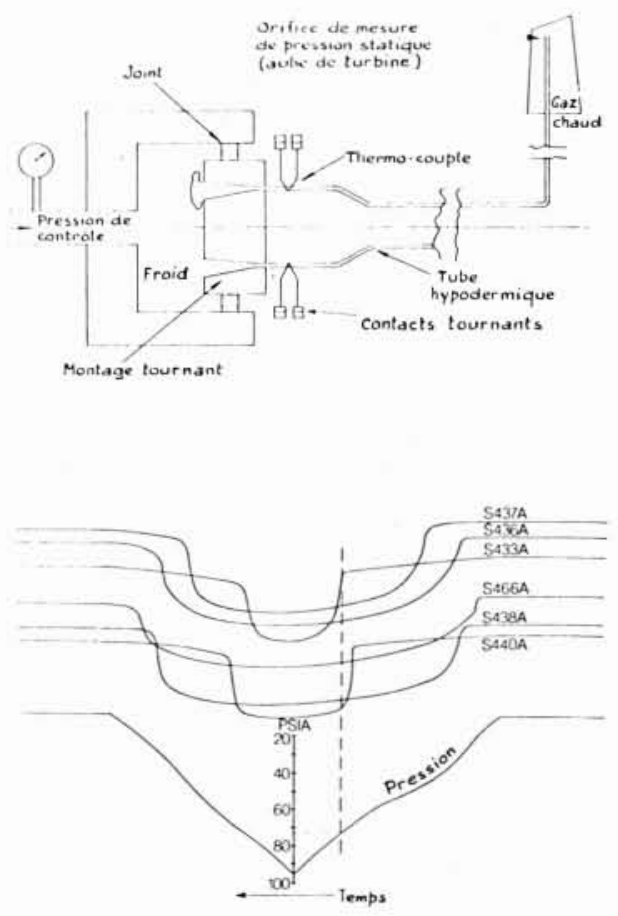

61. Schéma de principe du transmetteur " pineapple » et signaux types.

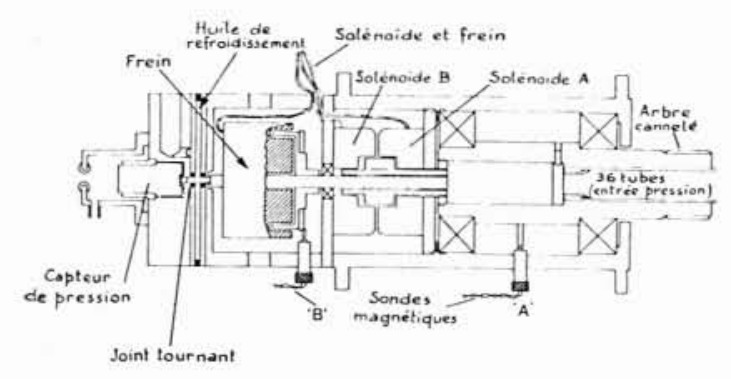

62. Scanivalve tournant.

\section{Transmission, acquisition et traitement des données}

Nous avons examiné jusqu'à présent les moyens de mesurer tel ou tel paramètre ou d'obtenir telle ou telle information. Dans le cas de machines tournantes, il serait toujours intéressant de pouvoir effectuer des mesures en écoulement relatif, ce qui nécessite des sondes ou appareils de mesure tournants et pose par conséquent le problème de la transmission de l'information.

A cet effet, diverses méthodes ont été développées au cours des années passées; nous allons les passer rapidement en revue :

a) un dispositif explorateur pour sonde tournante [7], dont la figure 57 montre le principe.

b) un turbocommutateur de pression [8] [9], pour transmission de mesures de pression effectuées sur aubes mobiles (figure 58).

c) des contacteurs tournants à balais ou à mercure dont la figure 59 montre un exemple [54].

d) des dispositifs de télémesure [54], dont la figure 60 montre un schéma de principe.

e) un transmetteur "pineapple" [55], dont la figure 61 montre le principe.

f) un scanivalve tournant [56], dont le principe est indiqué sur la figure 62 .

- Plusieurs dispositifs de télémesure ont également été développés à EDF/DTG [57].

En ce qui concerne le problème de l'acquisition et du traitement des données, c'est un problème trop vaste qui déborde du cadre de cette étude. De nombreuses solutions existent sur le plan industriel qui doivent tenir compte de certains impératifs d'application : nombre de voies, niveau, bande passante, type de stockage, type de traitement souhaité, etc.

Notons que quelques exemples de chaînes ont été indiqués précédemment et que d'autres figurent dans certains des documents indiqués en référence. 


\section{Conclusion}

Ce tour d'horizon sur les moyens de mesure développés au cours de ces dernières années est forcément incomplet car limité à certains domaines spécifiques. De même, l'aspect performance, contraintes d'utilisation, disponibilité du matériel, n'a pas été abordé pour ne pas gonfler exagérement cet article.
Néanmoins, il montre et c'est le but poursuivi, que les chercheurs, les laboratoires et les industriels disposent d'un large éventail de moyens d'investigation pour accéder à une meilleure et plus fine connaissance des phénomènes, que ce soit dans l'air comme dans l'eau.

Certains de ces moyens sont très puissants, mais aussi généralement très coûteux. Il importe donc de bien étudier tout projet de programme d'essais afin de définir le plus judicieusement possible les objectifs et les types de mesures s'y rapportant.

\section{Références}

[1] LAmbourion J., LARguier R. - Description, étalonnage et exemple d'utilisation d'un capteur de pression à court temps de réponse. Rech. Aérosp., n 133, 1969.

[2] Mallon J.R., KURTZ A.D. - Static performance of integrated senser transducers $25^{\text {th }}$ annual ISA Instrumentation Symposium May 79 Anaheim-Californie.

[3] Portat M. - Les capteurs pelliculaires et leurs applications. La Recherche aérospatiale, $\mathrm{n}^{\circ} 1982,3$.

[4] Bruere A., Portat M., Godefroy J.C., hellas F. Utilisation des capteurs pelliculaires pour la mesure sur turbomachines. AGARD/PEP: Instrumentation de pointe pour les composants des moteurs, Philadelphie 19-24 mai 1986.

[5] Chatanier M., Le Bot Y. - Utilisation des capteurs pelliculaires pour la mesure des pressions instationnaires dans les turbomachines. IUTAM - Symposium on Aeroelasticity in Turbomachines, Paris, Octobre 1976.

[6] Raffy P., Lewy S., Lambourion J., Chatanier M. Investigation of subsonic fan noise source by fluctuating pressure measurement on rotating blades. AIAA - Paper $\mathrm{n}^{\circ}$ 77-1321 - $4^{\text {th }}$ Aeroacoustic Conference - Atlanta - Octobre 1977.

[7] LE BOT Y., LARGUIER R. - Caractérisation expérimentale des écoulements internes dans les turbomachines. Revue Française de Mécanique, $\mathrm{n}^{\circ}$ 57, 1976, TP ONERA n 1976, 125.

[8] De Sievers A., Larguier R. - Dispositif d'étude de l'écoulement aérodynamique interne d'une turbomachine axiale. ATMA, Session 1973.

[9] LARGUier R. - Méthodes d'analyse expérimentale des écoulements instationnaires dans les turbomachines. ATMA, Session 1979.

[10] Catalogue DANTEC.

[11] Ligrani P., Gyles B. - Sensor development for on blade vector measurements. Lecture series: Techniques de mesures dans les turbomachines, mai $81-V K I-$ Bruxelles.

[12] Bertoglio J.P., Mathieu J., Charnay G. - Effects of Coriolis forces on the Turbulent Field inside Centrifugal Impellers. $5^{\text {th }}$ International Symposium on Air Breathing Engine, Février 81 - Bangalore - Inde.

[13] ELDER R. - Laser anemometer studies of the flow within a small high speed centrifugal compressor. Lecture series: Techniques de mesures dans les turbomachines, mai 81 - VKI - Bruxelles.
[14] JANSSENS G. - Ensemencement d'écoulements à température moyenne $\left(200\right.$ à $\left.300^{\circ} \mathrm{C}\right)$ en vue de mesures par vélocimétrie laser. RT ONERA $n^{\circ} 4 / 3467$ EN.

[15] SCHODL R. - Proceedings of the Agard Conference on Laser Optical Measurement Methods for aero Engine Research and Development LS 90-1977.

[16] Boutier A., Fertin G., Larguier R., Lefevre J., De Sievers A. - Laser anemometry applied to a research compressor Groupe de travail ISL-AGARD sur l'anémométrie laser Institut Franco-Allemand de St Louis, 5-7 mai 1976, TP ONERA $\mathrm{n}^{\circ} 1976,43$.

[17] KanNemans H. - Laser Doppler measurements in transparent pump impellers. Lecture serie: Techniques de mesures dans les turbomachines, mai 1981 VKI - Bruxelles.

[18] Melinand J.P., Charnay G. - Digital analysis of LDA Counter Signals in a Separated Boundary Layer. Proceedings of the Dynamic Flow Conference 1978, IMST - Marseille, septembre 1978.

[19] NAPIE P. - Sonde vélocimétrique pour écoulements anisotropes. Rapport interne EDF/DER, 1977, $\mathrm{n}^{\circ}$ P131D79.

[20] Dessus B., Lesne J.L., Napie P. - La mesure optique des vitesses par effet Doppler. Bulletin de la DER, $\mathrm{n}^{\circ}$ 1, 1978, série $A, 71$ pages.

[21] LESNE J.M. - Etude d'un anémomètre à laser. Thèse 1976 Université Paris XI.

[22] Document EDF/DER/DE/DTT. - Mesures de vitesse par corrélation.

[23] Document EDF/DER/DE/DTT. - Mesure de déplacements ou de vitesses par analyse d'images.

[24] GLAND M. - Mesures météorologiques par sondeur acoustique. Document EDF/DER/AEE EAA.

[25] Fiche EDF/DTG/n 73/1981. - Améliorations apportées aux mesures par moulinets en régimes permanents et transitoires.

[26] Demagny J.-CH. - Mesures de vitesses et de débits par la méthode électromagnétique - Choix des matériels industriels. La Houille Blanche, $\mathrm{n}^{\circ}$ 5-1976.

[27] DEMARAIS J.-C. - Débitmètre à ultrasons pour la mesure de débits liquides. Mesure - Régulation - Automatisme, vol. $43, \mathrm{n}^{\circ} 6 / 7$ - 1978, TP ONERA 1978, 86.

[28] Document EDF/DTG. - Expérience d'utilisation des débitmètres à ultrasons pour la mesure des débits transités par les turbines hydrauliques à EDF. 
[29] DEMARAIS J.C. - Débitmètre à ultrasons à large bande pour la mesure des débits instationnaires. RT ONERA n ${ }^{\circ} 10 / 3173$ EY 12/1985.

[30] Document Fischer Porter. - Débitmètre piézo-processif et convertisseur de mise en forme pour gaz et liquides.

[31] EDF/DER/DE. - Mesure des débits d'eau dans les conduites fermées par la méthode du temps de transit d'un traceur radiocatif. Techniques de mesures 0321 - Juillet 1982.

[32] EDF/DER. - Mesure du débit d'eau en conduite de grand diamètre par la méthode du temps de transit d'un traceur radioactif. Techniques de mesures 1036, janvier 1984.

[33] Fiche EDF/DTG - $\mathrm{n}^{\circ} 56$. - Mesure des températures en rivière. Thermomètre digital autonome.

[34] Godefroy J.-C. - Capteurs pelliculaires de température et de flux thermique. Recherche aérospatiale, $\mathrm{n}^{\circ}$ 1981/2.

[35] Jeunhomme L.B. - Application des fibres optiques aux mesures de température. Journées d'Etude: Mesures de températures, 16-17 déc. 86 Hilton, Paris.

[36] TARAN J.P., PEAlat M. - Practical CARS Temperature Measurement dans «Temperature, its Measurement and Control in Science and Industry ", 5, J.F. Schooley éditeur (1982) American Institute of Physics.

[37] Catalogue Artémis. - Nistadyn.

[38] EDF/DTG. - Limnimètre statique autonome. Fiche $\mathrm{n}^{\circ} 77$.

[39] MestCHERINOFF G. - La mesure industrielle de la turbidité. Mesure - Régulation - Automatisme, juin-juillet 1980.

[40] Document EDF/DSRE. - Stations de contróle de la qualité des eaux de rivière.

[41] Fiche EDF/DER/DEAA. - Indice de dégradation de la chlorophylle a phytoplanctonique.

[42] Document EDF/DER/DEAA. - L'ATP: méthode d'estimation de la biomasse algale et bactérienne.

[43] HAMElın P. - Application de la diffusion lumineuse à la métrologie des particules en écoulement diphasique dispersé. Thèse 1986, Université de Toulouse. Bulletin de la $D E R$, série $\mathrm{A}, \mathrm{n}^{\circ} 3 / 4,227$ pages.

[44] KLEITZ A. - Application de l'holographie ultra-rapide à la détermination de la taille et de la vitesse des particules dans un écoulement diphasé. La Houille Blanche, $\mathrm{n}^{\circ} 8-1973$, p. 677-687.

[45] Farmer W.-H. - Appl. Opt. 11, 2603 (1974) Appl. Opt., 13, 610 (1974).
[46] Sulle C., Courant J.-J. - Mesure de titre par calorimètre électrique, Rapport EDF, P32/023/22.

[47] LECOMTE J.-N. - In situ bubble sizing using a reference beam laser-Doppler anemometer - ISL - R 117/80. Procd. Symp. on long range and short range optical velocity measurements, sept 15-18, 1980, St Louis.

[48] Marie J.-L., Dumontier A., Lance M. - Application de l'anémométrie laser à effet Doppler à l'étude des écoulements diphasiques dispersés. $1 I^{*}$ Table Ronde LDA Université Paris XII - Créteil, mars 1981.

[49] Note EDF/DER/RNE/DAPE. - Mesure du taux de présence dans un faisceau tubulaire placé dans un écoulement diphasique à l'aide d'une sonde à fibre optique, mai 1981.

[50] Note EDF/DER/DTT. - Détermination du taux de fluctuations d'un écoulement diphasique.

[51] EDF/DER/DE. - Méthodes radioactives de mesure du taux de vide d'un écoulement diphasique. Fascicule 0112, novembre 1980.

[52] Cohen C., Lesne J.L. - La télédétection active en France; les développements laser pour les applications météorologiques OPTO 85 p. 147-150.

[53] Modern Methods of Testing Rotating. Components of Turbomachines. AGARD ograph $\mathrm{n}^{\circ} 207,1975$.

[54] DE Rose R. - Signal transmission via sliprings and telemetry. Lecture serie: Techniques de Mesures dans les Turbomachines, mai 1981, VKI, Bruxelles.

[55] WORTHY J.G.B. - The design, development and operation of gas turbine radio telemetry systems. Lecture Serie: Techniques de mesures dans les Turbomachines. Mai 1981, VKI, Bruxelles.

[56] CHIVERS J.N.H. - Blade pressure measurements. Lecture Serie: Techniques de mesures dans les turbomachines, mai 1981, VKI Bruxelles.

[57] EDF/DTG. - Notices - Télémesure sur machine tournante, juillet 74. Télémesure embarquée six canaux, juin 75. Dispositif de télémesure pour température rotor, décembre 75. Dispositif de télémesure courant-tension d'excitation, juin 78. Télémesure sur les rotors d'alternateurs, janvier 1982.

Nota : Plusieurs références concernent des rapports internes. Les lecteurs intéressés par ces documents sont priés de prendre contact avec l'auteur du présent article (J. Paulon, ONERA, Fort de Palaiseau, 91120 Palaiseau, France). 